\title{
A QUANTUM ANALOGUE OF THE FIRST FUNDAMENTAL THEOREM OF CLASSICAL INVARIANT THEORY
}

\author{
G. I. LEHRER, HECHUN ZHANG, AND R. B. ZHANG
}

\begin{abstract}
We establish a noncommutative analogue of the first fundamental theorem of classical invariant theory. For each quantum group associated with a classical Lie algebra, we construct a noncommutative associative algebra whose underlying vector space forms a module for the quantum group and whose algebraic structure is preserved by the quantum group action. The subspace of invariants is shown to form a subalgebra, which is finitely generated. We determine generators of this subalgebra of invariants and determine their commutation relations. In each case considered, the noncommutative modules we construct are flat deformations of their classical commutative analogues. Thus by taking the limit as $q \rightarrow 1$, our results imply the first fundamental theorem of classical invariant theory, and therefore generalise them to the noncommutative case.
\end{abstract}

\section{Contents}

1. Introduction

2. Generalities on module algebras over quantum groups

2.1. Module algebras

2.2. Braided symmetric algebras

2.3. A quantum analogue of the symmetric algebra

2.4. The quantum trace and invariants

3. Invariant theory of the quantum even orthogonal groups $\mathrm{U}_{q}\left(\mathfrak{s o}_{2 n}\right)$

3.1. The natural module for $\mathrm{U}_{q}\left(\mathfrak{s o}_{2 n}\right)$

3.2. The braided symmetric algebra of the natural $\mathrm{U}_{q}\left(\mathfrak{s o}_{2 n}\right)$-module

3.3. A $\mathrm{U}_{q}\left(\mathfrak{s o}_{2 n}\right)$ module algebra: $S_{q}(V)^{\otimes m}$ with twisted multiplication

3.4. Noncommutative FFT of invariant theory

4. Invariant theory of the quantum odd orthogonal groups $\mathrm{U}_{q}\left(\mathfrak{s o}_{2 n+1}\right)$

4.1. Braided symmetric algebra of the natural $\mathrm{U}_{q}\left(\mathfrak{s o}_{2 n+1}\right)$-module

4.2. $\mathrm{A} \mathrm{U}_{q}\left(\mathfrak{s o}_{2 n+1}\right)$-module algebra and the FFT of invariant theory

5. Invariant theory for the quantum symplectic groups $\mathrm{U}_{q}\left(\mathfrak{s p}_{2 n}\right)$

5.1. Braided symmetric algebra of the natural $\mathrm{U}_{q}\left(\mathfrak{s p}_{2 n}\right)$-module

5.2. $\mathrm{A} \mathrm{U}_{q}\left(\mathfrak{s p}_{2 n}\right)$-module algebra and the FFT of invariant theory

6. Invariant theory for the quantum general linear group

6.1. Algebra of functions on the quantum general linear group

6.2. Invariant theory for the quantum general linear group

6.3. Proof of the FFT for the quantum general linear group

6.4. The braided exterior algebra

Acknowledgement 


\section{INTRODUCTION}

The fundamental theorems of classical invariant theory may be formulated in three equivalent ways. Given a module $V$ for a reductive group $G$ over a field $k$, the first formulation provides a complete description of $\operatorname{End}_{k G}\left(V^{\otimes r}\right)$; the second does the same thing for the linear space of $G$-invariant multilinear functions $f: W \rightarrow k$, where $W=\oplus^{r} V \oplus^{s} V^{*}$, i.e. multilinear functions which are constant on $G$-orbits of $W$. Thirdly, the commutative algebra $S\left(W^{*}\right)$ of polynomial functions on $W$, is naturally a $G$-module, and one describes the subalgebra of invariant functions. In each case the 'first fundamental theorem' (FFT) provides generators (suitably defined) for the space of invariants, while the 'second fundamental theorem' (SFT) describes all relations among the generators. Although equivalent in principle, the statements of the fundamental theorems in their three formulations are not equally straightforward. For example, the SFT for orthogonal and symplectic groups in the first context would require a hitherto unknown description of an ideal in the Brauer algebras (cf. [19]).

A typical case addressed by the fundamental theorems is when $G$ is one of the classical groups over the complex numbers $\mathbb{C}$, and the $G$-module $V$ is the natural module. In these cases, the standard polynomial (third) form of the first fundamental theorem of classical invariant theory [30] yields a finite set of algebra generators for the subalgebra of invariants. In the first formulation given above, the fundamental theorems are often referred to as (generalised) Schur-Weyl-Brauer duality.

In this work we shall consider quantum analogues of the FFT in its third formulation. That is, we shall consider the action of the quantum groups corresponding to the classical Lie algebras on non-commutative analogues of the $S\left(W^{*}\right)$ above, and provide a finite set of generators of the (also generally non-commutative-see below) subalgebra of invariants. Note that the concept of "generators" of course depends on the structure of the algebra on which the quantum group acts, and that is a crucial aspect of this work. There is some work in this direction in the literature. A type of Howe duality between quantum general linear groups was constructed in [33], which implies the Jimbo-Schur-Weyl duality between the quantum general linear group and the Hecke algebra. Also the paper [28] investigated quantum analogues of polynomial invariants for the symplectic Lie algebra. The works [10, 11] have elements in common with our work, as does [4]. The work [22] provides a general context for Hopf algebra actions on non-commutative algebras. However, to our knowledge, there is no systematic treatment of the invariant theoretic aspects of the subject.

There is a considerable literature on endomorphism algebras of tensor powers of quantum group modules. In particular, when $\mathfrak{g}$ is a classical Lie algebra and $V$ is the natural module for the quantum group $\mathrm{U}_{q}(\mathfrak{g}$ ) (a slight enlargement of $\mathrm{U}_{q}(\mathfrak{g})$ is more convenient when $\left.\mathfrak{g}=\mathfrak{s o}_{2 n}\right)$, the algebra $\operatorname{End}_{\mathrm{U}_{q}(\mathfrak{g})}\left(V^{\otimes r}\right)$ at generic $q$ is known to be a quotient of the Hecke algebra of type $A$ if $\mathfrak{g}=\mathfrak{g l}_{n}$ and a quotient of a Birman-Wenzl-Murakami algebra in the other cases [16, 24, 17, 9, 18]. The duality between the quantum general linear group and Hecke algebra is known to be valid even when $q$ is a root of unity [9]. In [18], the algebras $\operatorname{End}_{\mathrm{U}_{q}(\mathfrak{g})}\left(V^{\otimes r}\right)$, where $V$ is the 7-dimensional irreducible module for $\mathrm{U}_{q}\left(G_{2}\right)$ or any finite dimensional irreducible module for $\mathrm{U}_{q}\left(\mathfrak{s l}_{2}\right)$ were described in terms of representations of the Artin 
braid group. The endomorphism algebras for tensor powers of certain irreducible representations of the $E$ series of quantum groups were studied by Wenzl [29].

The general framework for developing a noncommutative analogue of classical invariant theory for quantum groups is well established in the setting of arbitrary Hopf algebras [22] (see also [4]). Let $\mathrm{U}_{q}(\mathfrak{g})$ be a quantum group, and let $A$ be a module algebra over $\mathrm{U}_{q}(\mathfrak{g})$ in the sense of [22, $\left.\S 4.1\right]$; that is, $A$ is an associative algebra whose underlying vector space is a $\mathrm{U}_{q}(\mathfrak{g})$-module, and whose algebraic structure is preserved by the quantum group action. Then the subspace $A^{\mathrm{U}_{q}(\mathfrak{g})}$ of invariants in $A$ is a subalgebra. Our aim is to describe the algebraic structure of $A^{\mathrm{U}_{q}(\mathfrak{g})}$.

Corresponding to each classical Lie algebra $\mathfrak{g}$, we construct an associative algebra, which is a module algebra for the quantum group $\mathrm{U}_{q}(\mathfrak{g})$ and reduces in the limit $q \rightarrow 1$ to the polynomial algebra over a direct sum of copies of the natural $\mathfrak{g}$-module (and its dual if $\mathfrak{g}$ is type $A$ ). Since the usual tensor product of $\mathrm{U}_{q}(\mathfrak{g})$ module algebras is not generally a module algebra, this construction makes essential use of the braiding of the quantum group given by the universal $R$-matrix, which does not appear in the setting of general Hopf algebras [22]. The question of a module algebra structure on tensor products has also been studied in [3, 34] and will be discussed below.

We then study the subspace of quantum group invariants in the module algebra. We shall show that the subspace of invariants always forms a subalgebra of the module algebra, and find a set of generators for the subalgebra of invariants together with the commutation relations obeyed by these generators. This result may be regarded as the FFT of the noncommutative analogue of classical invariant theory for quantum groups associated with the classical Lie algebras, in the third of the three formulations described above.

In all the cases studied, we find that the subalgebras of invariants are finitely generated. We prove this by explicit analysis of the subalgebras of invariants using the diagrammatical method of [25, 26]. We should point out that since a quantum group $\mathrm{U}_{q}(\mathfrak{g})$ is a non-cocommutative Hopf algebra, the relevant module algebras are noncommutative, and so also are their subalgebras of invariants. For this reason, most of the techniques in classical invariant theory, based on commutative algebra, do not apply here. In particular the general proofs of finite generation by Hilbert, Weyl and Nagata in the commutative context do not generalise to the quantum group setting.

Returning to the problem of constructing appropriate quantum analogues of polynomial algebras over quantum group modules, we note that it has long been known that there is no good quantum analogue of the coordinate ring of the 4-dimensional irreducible $\mathrm{U}_{q}\left(\mathfrak{s l}_{2}\right)$-module [27]. Recently Berenstein and Zwicknagl [3, 34] have carried out a systematic study of the braided symmetric algebras, which might be construed as quantum analogues of coordinate rings. They found that almost all the braided symmetric algebras are 'smaller' than the corresponding polynomial algebras thus are not suitable quantum analogues (e.g. in the sense of [27, Definition $2]$ ) of the latter. That is, they are not flat deformations of the coordinate rings. The same is true even of the braided symmetric algebras on direct sums of copies of the natural module of quantum groups of type $B, C$ and $D$. This may be a partial 
explanation of the lack of results available on quantum analogues of classical invariant theory in the third (polynomial function) formulation of the three described above. Our construction of quantum analogues of polynomial algebras will follow an approach suggested in [5], and the resulting algebras are not braided symmetric algebras in the sense of [3, 34].

There is a connection between the present work and noncommutative geometry [6]. The relationship between quantum groups and noncommutative geometry is well described in [21]. This suggests that quantum groups play much the same role in noncommutative geometry as that played by Lie groups in ordinary differential geometry. Indeed a noncommutative space $X$ is specified by its associative algebra $\mathcal{A}(X)$ of functions [6]. If $\mathcal{A}(X)$ is a module algebra over some quantum group $\mathrm{U}_{q}(\mathfrak{g})$, then we may regard $X$ as having a $\mathrm{U}_{q}(\mathfrak{g})$ symmetry algebra. Typical examples of such noncommutative geometries are the quantum homogeneous spaces studied in [12]. In order to understand the noncommutative space $X$, it is useful to determine the $\mathrm{U}_{q}(\mathfrak{g})$-modules structure of $\mathcal{A}(X)$, in particular, the subalgebra of invariants. We shall realise this in Lemma 3.5 and Corollary 4.3, for the quantum sphere.

This paper is organised as follows. Section 2 discusses generalities concerning module algebras for quantum groups. A crucial result is Theorem 2.3, which defines a twisted multiplication on the tensor product of any two module algebras by using the universal $R$-matrix of the quantum group to endow the tensor product with the structure of a module algebra. Sections 3 and 4 treat the invariant theory of the orthogonal quantum groups. The main results are summarised in Theorems 3.10 and 4.7, which provide a noncommutative FFT's of invariant theory for the quantum orthogonal groups. The subalgebra of invariants is noncommutative in the quantum setting, and we describe the commutation relations among the (finite set of) generators of the subalgebra of invariants in Lemmas 3.8 and 4.6. Section 5 treats the invariant theory of the symplectic quantum groups. The noncommutative FFT for the quantum symplectic groups is Theorem 5.6, and the commutation relations among the generators of the noncommutative subalgebra of invariants are given in Proposition 5.5. In Section 6, we study the invariant theory of the quantum general linear group. The techniques used in this section are similar to those of [12] but rather different from those in the previous sections. Theorem 6.10 provides a noncommutative FFT, and Lemma 6.9 describes the commutation relations among the generators of the subalgebra of invariants. In Theorem 6.16 we prove a quantum analogue of the skew $\left(\mathrm{GL}_{m}, \mathrm{GL}_{n}\right)$ duality [14, Theorem 4.1.1] of Howe.

Finally, we note that an alternative approach to the questions addressed here may be to start with the study of endomorphisms of tensor powers (the first formulation), and translate versions of the first and second fundamental theorems to the other two settings. One might even hope to apply methods such as those in the appendix of [2] to move from type $A$ to the other classical types. This strategy seems to provide a route towards a SFT in the quantum setting, and we intend to return to this theme in a future work.

\section{Generalities on module algebras over quantum Groups}

2.1. Module algebras. For a simple complex Lie algebra $\mathfrak{g}$, we denote by $\mathrm{U}_{q}(\mathfrak{g})$ the associated quantum group over the field of rational functions $\mathcal{K}=\mathbb{C}\left(q^{\frac{1}{2}}\right)$ where $q$ 
is an indeterminate. The algebra $\mathrm{U}_{q}(\mathfrak{g})$ has a standard presentation with generators $e_{i}, f_{i}, k_{i}^{ \pm 1}(1 \leq i \leq \operatorname{rank}(\mathfrak{g}))$ and the usual relations (see, e.g. [15, Ch. 4], whose conventions we do not follow precisely, but which is a good general reference). It is well known that $\mathrm{U}_{q}(\mathfrak{g})$ is a Hopf algebra. Write $\Delta, \epsilon$ and $S$ for the co-multiplication, co-unit and the antipode respectively. We use the following convention in defining co-multiplication:

$$
\begin{aligned}
& \Delta\left(e_{i}\right)=e_{i} \otimes k_{i}+1 \otimes e_{i}, \\
& \Delta\left(f_{i}\right)=f_{i} \otimes 1+k_{i}^{-1} \otimes f_{i}, \\
& \Delta\left(k_{i}\right)=k_{i} \otimes k_{i} .
\end{aligned}
$$

If $\mathfrak{g}$ is a semi-simple Lie algebra, or a direct sum of general linear algebras, we denote by $\mathrm{U}_{q}(\mathfrak{g})$ the tensor product of the quantum groups of the components of $\mathfrak{g}$. The definition of the quantum general linear group $\mathrm{U}_{q}\left(\mathfrak{g l}_{n}\right)$ will be given in Section 6.1.

An important structural property of a quantum group is its braiding, namely, the existence of a universal $R$-matrix. We may think of $R$ as an invertible element in some appropriate completion of $\mathrm{U}_{q}(\mathfrak{g}) \otimes \mathrm{U}_{q}(\mathfrak{g})$, whose action on the tensor product of any two finite dimensional $\mathrm{U}_{q}$-modules of type $(1, \ldots, 1)$ is well defined. It satisfies the following relations

$$
\begin{aligned}
R \Delta(x) & =\Delta^{\prime}(x) R, \quad \forall x \in \mathrm{U}_{q}(\mathfrak{g}), \\
(\Delta \otimes \mathrm{id}) R & =R_{13} R_{23}, \quad(\mathrm{id} \otimes \Delta) R=R_{13} R_{12},
\end{aligned}
$$

where $\Delta^{\prime}$ is the opposite co-multiplication. Here the subscripts of $R_{13}$ etc. have the usual meaning as in [8]. It follows from (2.2) that $R$ satisfies the celebrated Yang-Baxter equation

$$
R_{12} R_{13} R_{23}=R_{23} R_{13} R_{12} .
$$

Remark 2.1. Note that if $V_{1}, V_{2}$ are $\mathrm{U}_{q}$-modules and $P: V_{1} \otimes V_{2} \rightarrow V_{2} \otimes V_{1}$ interchanges the factors of a tensor, then in terms of the operator $\check{R}:=P R$, (2.1) reads $\check{R} \Delta(x)=\Delta(x) \check{R}$, and (2.3) becomes the braid relation $\check{R}_{1} \check{R}_{2} \check{R}_{1}=\check{R}_{2} \check{R}_{1} \check{R}_{2}$.

Because of the Hopf algebra structure, the tensor product $V_{1} \otimes_{\mathcal{K}} V_{2}$ of any two $\mathrm{U}_{q}(\mathfrak{g})$-modules $V_{1}$ and $V_{2}$ becomes a $\mathrm{U}_{q}(\mathfrak{g})$-module. In view of 2.1, the $R$-matrix (which is invertible) defines an isomorphism of $\mathrm{U}_{q}(\mathfrak{g})$-modules, $V_{1} \otimes V_{2} \stackrel{\check{R}}{\longrightarrow} V_{2} \otimes V_{1}$.

We shall make extensive use of the notion of module algebras over a Hopf algebra [22, §4.1]. An associative algebra $(A, \mu)$ with multiplication $\mu$ and identity element 1 is a $\mathrm{U}_{q}(\mathfrak{g})$-module algebra if $A$ is a $\mathrm{U}_{q}(\mathfrak{g})$-module such that the $\mathrm{U}_{q}(\mathfrak{g})$-action preserves the algebraic structure of $A$, that is,

$$
x \mu(a \otimes b)=\sum_{(x)} \mu\left(x_{(1)}(a) \otimes x_{(2)}(b)\right), \quad x(1)=\epsilon(x) 1,
$$

for all $a, b \in A$ and $x \in \mathrm{U}_{q}(\mathfrak{g})$.

We shall sometimes use the term $\mathrm{U}_{q}(\mathfrak{g})$-algebra as a synonym for $\mathrm{U}_{q}(\mathfrak{g})$-module algebra.

Let $A^{\mathrm{U}_{q}(\mathfrak{g})}=\left\{t \in A \mid x(t)=\epsilon(x) t, \forall x \in \mathrm{U}_{q}(\mathfrak{g})\right\}$ be the subspace of $\mathrm{U}_{q}(\mathfrak{g})$ invariants in the module algebra $A$. The following result is well known.

Lemma 2.2. Let $A$ be a $\mathrm{U}_{q}(\mathfrak{g})$-algebra. Then the subspace $A^{\mathrm{U}_{q}(\mathfrak{g})}$ of invariants is a subalgebra of $A$. 
Proof. Using Sweedler's notation, write the co-multiplication in $\mathrm{U}_{q}(\mathfrak{g})$ as $\Delta(x)=$ $\sum_{(x)} x_{(1)} \otimes x_{(2)}$. Then for all $t, t^{\prime} \in A^{\mathrm{U}_{q}(\mathfrak{g})}$,

$$
x\left(t t^{\prime}\right)=\sum x_{(1)}(t) x_{(2)}\left(t^{\prime}\right)=\sum \epsilon\left(x_{(1)}\right) \epsilon\left(x_{(2)}\right) t t^{\prime}=\epsilon(x) t t^{\prime}, \quad \forall x \in \mathrm{U}_{q}(\mathfrak{g}) .
$$

Thus $t t^{\prime} \in A^{\mathrm{U}_{q}(\mathfrak{g})}$.

We remark that the above result is valid for arbitrary Hopf algebras.

A module algebra $A$ is called locally finite if $\mathrm{U}_{q}(\mathfrak{g}) a$ is finite dimensional for any $a \in A$, and is said to be of type $(1,1, \ldots, 1)$ if $A$ is of type $(1,1, \ldots, 1)$ as a $\mathrm{U}_{q}(\mathfrak{g})$ module. Recall that locally finite $\mathrm{U}_{q}(\mathfrak{g})$-modules are semi-simple (see e.g., [1]).

Theorem 2.3. Let $\left(A, \mu_{A}\right)$ and $\left(B, \mu_{B}\right)$ be locally finite $\mathrm{U}_{q}(\mathfrak{g})$-module algebras of type $(1,1, \ldots, 1)$. Then $A \otimes B$ acquires a $\mathrm{U}_{q}(\mathfrak{g})$-module algebra structure when endowed with the following multiplication

$$
\mu_{A, B}=\left(\mu_{A} \otimes \mu_{B}\right)\left(i d_{A} \otimes P R \otimes i d_{B}\right),
$$

where $R$ is the universal $R$-matrix of $\mathrm{U}_{q}(\mathfrak{g})$, and $P: B \otimes A \longrightarrow A \otimes B$ is the permutation map defined by $a \otimes b \mapsto b \otimes a$.

Proof. This result was stated in [5] and is probably well known. We give a proof here because it is central to this work. Write the universal $R$-matrix of $\mathrm{U}_{q}(\mathfrak{g})$ as $R=\sum \alpha_{t} \otimes \beta_{t}$, where $\alpha_{t}, \beta_{t}$ may be taken to be elements of $\mathrm{U}_{q}(\mathfrak{g})$. Then for $a_{i} \in A$ and $b_{i} \in B(i=1,2,3)$, we have

$$
\begin{aligned}
& \left(\left(a_{1} \otimes b_{1}\right)\left(a_{2} \otimes b_{2}\right)\right)\left(a_{3} \otimes b_{3}\right) \\
& =\left(\sum_{t} a_{1} \beta_{t}\left(a_{2}\right) \otimes \alpha_{t}\left(b_{1}\right) b_{2}\right)\left(a_{3} \otimes b_{3}\right) \\
& \left.=\sum_{t, s} a_{1} \beta_{t}\left(a_{2}\right) \beta_{s}\left(a_{3}\right) \otimes \alpha_{s}\left(\alpha_{t}\left(b_{1}\right) b_{2}\right)\right) b_{3} .
\end{aligned}
$$

Using the first relation of (2.2), we can re-write the last expression as

$$
\sum_{t, s, r} a_{1} \beta_{t}\left(a_{2}\right)\left(\beta_{s} \beta_{r}\left(a_{3}\right)\right) \otimes\left(\alpha_{s} \alpha_{t}\left(b_{1}\right)\right) \alpha_{r}\left(b_{2}\right) b_{3} .
$$

Then using the second relation of (2.2), we can re-write this as

$$
\begin{aligned}
& \sum_{r, s} a_{1} \beta_{s}\left(a_{2} \beta_{r}\left(a_{3}\right)\right) \otimes \alpha_{s}\left(b_{1}\right) \alpha_{r}\left(b_{2}\right) b_{3} \\
& =\left(a_{1} \otimes b_{1}\right) \sum_{r} a_{2} \beta_{r}\left(a_{3}\right) \otimes \alpha_{r}\left(b_{2}\right) b_{3} \\
& =\left(a_{1} \otimes b_{1}\right)\left(\left(a_{2} \otimes b_{2}\right)\left(a_{3} \otimes b_{3}\right)\right) .
\end{aligned}
$$

This proves the associativity of the multiplication. 
The action of $\mathrm{U}_{q}(\mathfrak{g})$ on $A \otimes B$ is defined in the obvious way: for all $a \otimes b \in A \otimes B$ and $x \in \mathrm{U}_{q}(\mathfrak{g}), x(a \otimes b):=\sum_{(x)} x_{(1)}(a) \otimes x_{(2)}(b)$. Now

$$
\begin{aligned}
x\left(\left(a_{1} \otimes b_{1}\right)\left(a_{2} \otimes b_{2}\right)\right) & =x\left(\sum_{t} a_{1} \beta_{t}\left(a_{2}\right) \otimes \alpha_{t}\left(b_{1}\right) b_{2}\right) \\
& =\sum_{t} \sum_{(x)} x_{(1)}\left(a_{1} \beta_{t}\left(a_{2}\right)\right) \otimes x_{(2)}\left(\alpha_{t}\left(b_{1}\right) b_{2}\right) \\
& =\sum_{t} \sum_{(x)} x_{(1)}\left(a_{1}\right) x_{(2)}\left(\beta_{t}\left(a_{2}\right)\right) \otimes x_{(3)}\left(\alpha_{t}\left(b_{1}\right)\right) x_{(4)}\left(b_{2}\right) .
\end{aligned}
$$

Using (2.1), we can re-write this last expression as

$$
\begin{aligned}
& \sum_{t} \sum_{(x)} x_{(1)}\left(a_{1}\right) \beta_{t}\left(x_{(3)}\left(a_{2}\right)\right) \otimes \alpha_{t}\left(x_{(2)}\left(b_{1}\right)\right) x_{(4)}\left(b_{2}\right) \\
= & \sum_{(x)}\left(x_{(1)}\left(a_{1}\right) \otimes x_{(2)}\left(b_{1}\right)\right)\left(x_{(3)}\left(a_{2}\right) \otimes x_{(4)}\left(b_{2}\right)\right) \\
= & \sum_{(x)} x_{(1)}\left(a_{1} \otimes b_{1}\right) x_{(2)}\left(a_{2} \otimes b_{2}\right) .
\end{aligned}
$$

This proves that the $\mathrm{U}_{q}(\mathfrak{g})$ action on $A \otimes B$ preserves the multiplication.

Next, write $1_{A}, 1_{B}$ for the identity elements of $A, B$ respectively. A short calculation using the equations $\sum_{t} \epsilon\left(\alpha_{t}\right) \beta_{t}=1$ and $\sum_{t} \epsilon\left(\beta_{t}\right) \alpha_{t}=1$ shows that $1_{A} \otimes 1_{B}$ is the identity element of $A \otimes B$. Moreover we have $x(1 \otimes 1)=\epsilon(x) 1 \otimes 1$ for all $x \in \mathrm{U}_{q}(\mathfrak{g})$. This completes the proof of the theorem.

Remark 2.4. Henceforth all $\mathrm{U}_{q}(\mathfrak{g})$-modules will be assumed to be locally finite of type $(1,1, \ldots, 1)$, as will all $\mathrm{U}_{q}(\mathfrak{g})$-module algebras.

The next two results prove the basic properties of the tensor product construction. The first proves the associativity of the tensor product on the category of $\mathrm{U}_{q}(\mathfrak{g})$ algebras.

Lemma 2.5. Let $A, B$ and $C$ be three $\mathrm{U}_{q}(\mathfrak{g})$-algebras. The module algebras $(A \otimes$ $\left.B \otimes C, \mu_{A \otimes B, C}\right)$ and $\left(A \otimes B \otimes C, \mu_{A, B \otimes C}\right)$ are equal.

Proof. We need to show that the multiplication maps $\mu_{A \otimes B, C}$ and $\mu_{A, B \otimes C}$ from $(A \otimes B \otimes C)^{\otimes 2}$ to $A \otimes B \otimes C$ are identical. For all $a \otimes b \otimes c$ and $a^{\prime} \otimes b^{\prime} \otimes c^{\prime}$ in $A \otimes B \otimes C$, we have

$$
\begin{aligned}
& \mu_{A \otimes B, C}\left((a \otimes b \otimes c) \otimes\left(a^{\prime} \otimes b^{\prime} \otimes c\right)\right) \\
& =\sum_{t} \mu_{A, B}\left((a \otimes b) \otimes \beta_{t}\left(a^{\prime} \otimes b^{\prime}\right)\right) \otimes \alpha_{t}(c) c^{\prime} \\
& =\sum_{r, s, t} a\left(\beta_{r} \beta_{t}\left(a^{\prime}\right)\right) \otimes \alpha_{r}(b) \beta_{s}\left(b^{\prime}\right) \otimes \alpha_{s} \alpha_{t}(c) c^{\prime} \\
& \mu_{A, B \otimes C}\left((a \otimes b \otimes c) \otimes\left(a^{\prime} \otimes b^{\prime} \otimes c\right)\right) \\
& =\sum_{t} a \beta_{t}\left(a^{\prime}\right) \otimes \mu_{B, C}\left(\alpha_{t}(b \otimes c) \otimes\left(b^{\prime} \otimes c^{\prime}\right)\right) \\
& =\sum_{r, s, t} a\left(\beta_{r} \beta_{t}\left(a^{\prime}\right)\right) \otimes \alpha_{r}(b) \beta_{s}\left(b^{\prime}\right) \otimes \alpha_{s} \alpha_{t}(c) c^{\prime} .
\end{aligned}
$$


Hence $\mu_{A \otimes B, C}=\mu_{A, B \otimes C}$.

Remark 2.6. Lemma 2.5 shows that given $\mathrm{U}_{q}(\mathfrak{g})$-algebras $A_{i}(i=1,2, \ldots, k)$, one can iterate the construction of Theorem 2.3 to obtain an unambiguous $\mathrm{U}_{q}(\mathfrak{g})$-module algebra structure on $A_{1} \otimes A_{2} \otimes \cdots \otimes A_{k}$.

The next statement sets out the key properties of homomorphisms of $\mathrm{U}_{q}(\mathfrak{g})$ algebras in the tensor category.

Proposition 2.7. Let $\alpha: A \rightarrow A^{\prime}$ and $\beta: B \rightarrow B^{\prime}$ be homomorphisms of $\mathrm{U}_{q}(\mathfrak{g})$ algebras. Then

(1) The kernel and image of $\alpha$ (and $\beta$ ) are $\mathrm{U}_{q}(\mathfrak{g})$-algebras.

(2) The map $\alpha \otimes \beta: A \otimes B \longrightarrow A^{\prime} \otimes B^{\prime}$ is a homomorphism of $\mathrm{U}_{q}(\mathfrak{g})$-algebras.

Proof. The first assertion is clear.

To verify the second, we need to show that $\alpha \otimes \beta$ respects the multiplication, and the $\mathrm{U}_{q}(\mathfrak{g})$-action. Take elements $a_{1}, a_{2} \in A$ and $b_{1}, b_{2} \in B$, and write the $R$-matrix $R$ as $R=\sum_{t} r_{t} \otimes s_{t}$, with $r_{t}, s_{t} \in \mathrm{U}_{q}(\mathfrak{g})$. Then $\alpha \otimes \beta\left(a_{1} \otimes b_{1} . a_{2} \otimes b_{2}\right)$ is equal to $\sum_{t} \alpha\left(a_{1}\right) \cdot s_{t} \alpha\left(a_{2}\right) \otimes r_{t} \beta\left(b_{1}\right) . \beta\left(b_{2}\right)$. It is straightforward to check that this is equal to the product $\alpha\left(a_{1}\right) \otimes \beta\left(b_{1}\right) . \alpha\left(a_{2}\right) \otimes \beta\left(b_{2}\right)$ in $A^{\prime} \otimes B^{\prime}$, which shows that $\alpha \otimes \beta$ respects algebra multiplication.

Finally, if $u \in \mathrm{U}_{q}(\mathfrak{g})$ and $\Delta(u)=\sum_{i} u_{i} \otimes u_{i}^{\prime}$, then for elements $a \in A$ and $b \in B$, we have $\alpha \otimes \beta(u . a \otimes b)=\sum_{i} \alpha\left(u_{i} a\right) \otimes \beta\left(u_{i}^{\prime} b\right)=\sum_{i} u_{i} \alpha(a) \otimes u_{i}^{\prime} \beta(b)=u . \alpha(a) \otimes \beta(b)$. Hence $\alpha \otimes \beta$ respects the $\mathrm{U}_{q}$-action.

2.2. Braided symmetric algebras. The first step in our construction of the noncommutative analogues of coordinate rings with which we shall work is to define, for each finite dimensional $\mathrm{U}_{q}(\mathfrak{g})$-module $V$, a braided symmetric algebra $S_{q}(V)$; these algebras have been considered previously, e.g. in [3, §2.1].

Write $R_{V V} \in G L(V \otimes V, \mathcal{K})$ for the $R$-matrix associated to $\mathrm{U}_{q}(\mathfrak{g})$. Let $P$ : $V \otimes V \longrightarrow V \otimes V$, be the permutation map $v \otimes w \mapsto w \otimes v$, and define $\check{R}:=P R_{V, V}$. Then $\check{R} \in \operatorname{End}_{\mathrm{U}_{q}(\mathfrak{g})}(V \otimes V)$, and

$$
\left(\check{R} \otimes \mathrm{id}_{V}\right)\left(\mathrm{id}_{V} \otimes \check{R}\right)\left(\check{R} \otimes \mathrm{id}_{V}\right)=\left(\mathrm{id}_{V} \otimes \check{R}\right)\left(\check{R} \otimes \mathrm{id}_{V}\right)\left(\mathrm{id}_{V} \otimes \check{R}\right) .
$$

It follows from [18, Theorem 6.2] that $\check{R}^{2}$ acts on each isotypic component of $V \otimes V$ as a scalar of the form $q^{m}$, where $m \in \mathbb{Z}$. Hence $\check{R}$ is semisimple on $V \otimes V$, and has characteristic polynomial of the form (cf. [18, (6.10)])

$$
\prod_{i=1}^{k_{+}}\left(t-q^{\chi_{i}^{(+)}}\right) \prod_{j=1}^{k_{-}}\left(t+q^{\chi_{i}^{(-)}}\right)
$$

where $k_{ \pm}$are positive integers and $\chi_{i}^{(+)}$and $\chi_{i}^{(-)}$are rational numbers (in fact $\in \frac{1}{2} \mathbb{Z}$ ). Define submodules $a_{2}$ and $s_{2}$ of $V \otimes V$ by

$$
a_{2}=\prod_{i=1}^{k_{+}}\left(\check{R}-q^{\chi_{i}^{(+)}}\right)(V \otimes V), \quad s_{2}=\prod_{j=1}^{k_{-}}\left(\check{R}+q^{\chi_{i}^{(-)}}\right)(V \otimes V) .
$$

Let $T(V)$ be the tensor algebra of $V$. This is a $\mathrm{U}_{q}(\mathfrak{g})$-module in the obvious way, and the $\mathrm{U}_{q}(\mathfrak{g})$ action preserves the algebraic structure of $T(V)$; that is, $T(V)$ is a $\mathrm{U}_{q}(\mathfrak{g})$ module algebra. Let $\mathcal{I}_{q}$ be the two-sided ideal of $T(V)$ generated by $a_{2}$; since 
$\check{R}$ commutes with $\mathrm{U}_{q}(\mathfrak{g})$, this is stable under $\mathrm{U}_{q}(\mathfrak{g})$. Define the braided symmetric algebra $S_{q}(V)$ of the $\mathrm{U}_{q}(\mathfrak{g})$-module $V$ by

$$
S_{q}(V)=T(V) / \mathcal{I}_{q}
$$

Let $\tau: T(V) \longrightarrow S_{q}(V)$ be the natural surjection. Since the ideal $\mathcal{I}_{q}$ is a $\mathrm{U}_{q^{-}}$ subalgebra of $T(V)$, it follows from Proposition 2.7 that $S_{q}(V)$ is a module algebra over $\mathrm{U}_{q}(\mathfrak{g})$.

Note that the two-sided ideal $\left\langle a_{2}\right\rangle$ of $T(V)$ is a $\mathbb{Z}_{+}$-graded submodule of the $\mathbb{Z}_{+^{-}}$ graded module $T(V)$. Hence the quotient $S_{q}(V)$ is $\mathbb{Z}_{+}$-graded, and we shall denote by $S_{q}(V)_{n}$ its degree $n$ homogeneous component.

The symmetric algebra $S(V)$ of $V$ over $\mathcal{K}$ is also $\mathbb{Z}_{+}$-graded. Following [3], $S_{q}(V)$ is called a flat deformation of $S(V)$ if $\operatorname{dim} S_{q}(V)_{n}=\operatorname{dim} S(V)_{n}$ for all $n$. This happens if and only if $\operatorname{dim} S_{q}(V)_{3}=\operatorname{dim} S(V)_{3}$ by a result of Drinfeld's [8]. In this case, we shall also say that the $\mathrm{U}_{q}(\mathfrak{g})$-module $V$ itself is flat.

The results of [3, 34] show that flat modules of quantum groups are extremely rare. Other than the natural modules for the quantum groups associated with the classical Lie algebras, there are hardly any modules which are flat. Even in the case of $\mathrm{U}_{q}\left(\mathfrak{s l}_{2}\right)$, all the irreducible modules of dimensions greater than 3 are not flat!

To develop an analogue of classical invariant theory for quantum groups, we require quantum analogues of polynomial algebras on direct sums of copies of a given module. However even when a module is flat, the direct sum of several copies of it is usually not flat. For example the natural module $V$ of $\mathrm{U}_{q}\left(\mathfrak{s o}_{n}\right)$, is known to be flat 34] (see also below). Let $\oplus^{m} V$ denote the direct sum of $m$ copies of $V$. It is easily verified that $S_{q}\left(\oplus^{m} V\right)$ is not flat if $m>1$, and thus is not a suitable quantum analogue of $S\left(\oplus^{m} V\right)$.

2.3. A quantum analogue of the symmetric algebra. To construct a quantum analogue of $S\left(\oplus^{k} V\right)$, we shall use Theorem 2.3. Given a module $V$ for a quantum group $\mathrm{U}_{q}(\mathfrak{g})$, we have the braided symmetric algebra $S_{q}(V)$ as above. Iterating the construction of Theorem 2.3 , we obtain the $\mathrm{U}_{q}(\mathfrak{g})$-module algebra

$$
\mathcal{A}_{m}(V):=\left(S_{q}(V)^{\otimes m}, \mu_{S}\right) .
$$

Whenever there is no danger of confusion, we shall drop the $V$ from the notation and simply write $\mathcal{A}_{m}$ for $\mathcal{A}_{m}(V)$. This is a $\mathbb{Z}_{+}^{m}$-graded $\mathrm{U}_{q}$-algebra, with homogeneous component $\left(\mathcal{A}_{m}\right)_{\mathbf{d}}$ of multi-degree $\mathbf{d}=\left(d_{1}, d_{2}, \ldots, d_{m}\right)$ given by

$$
\left(\mathcal{A}_{m}\right)_{\mathbf{d}}=S_{q}(V)_{d_{1}} \otimes S_{q}(V)_{d_{2}} \otimes \cdots \otimes S_{q}(V)_{d_{m}} .
$$

If $V$ is flat, then $\operatorname{dim} S_{q}(V)_{k}=\operatorname{dim} S(V)_{k}$ for all $k$. In this case,

$$
\operatorname{dim} \sum_{|\mathbf{d}|=k}\left(\mathcal{A}_{m}\right)_{\mathbf{d}}=\operatorname{dim} S\left(\oplus^{m} V\right)_{k}, \quad|\mathbf{d}|=\sum_{i=1}^{m} d_{i} .
$$

This proves the following statement.

Theorem 2.8. If $V$ is a flat $\mathrm{U}_{q}(\mathfrak{g})$-module, the $\mathrm{U}_{q}(\mathfrak{g})$-module algebra $\mathcal{A}_{m}(V)$ defined by (2.6) is a flat deformation (in the sense of [3]) of the symmetric algebra $S\left(\oplus^{m} V\right.$ ) of the direct sum of $m$-copies of $V$. 
Let $\left\{v_{s} \mid s=1,2, \ldots, \operatorname{dim} V\right\}$ be a basis for the $\mathrm{U}_{q}(\mathfrak{g})$-module $V$. Since the natural map $\tau: T(V) \longrightarrow S_{q}(V)$ maps $T(V)_{1}=V$ injectively into $S_{q}(V)$, we also denote by $v_{s}$ the image of this basis element in $S_{q}(V)$. We introduce the following elements

$$
X_{i a}:=\underbrace{1 \otimes \cdots \otimes 1}_{i-1} \otimes v_{a} \otimes \underbrace{1 \otimes \cdots \otimes 1}_{m-i}
$$

of $\mathcal{A}_{m}$. Then for all $i<j$,

$$
X_{j a} X_{i b}=\sum_{a^{\prime}, b^{\prime}=1}^{2 n} \check{R}_{a^{\prime} a, b^{\prime} b} X_{i a^{\prime}} X_{j b^{\prime}} .
$$

where $\check{R}_{a a^{\prime}, b b^{\prime}}$ are the entries of the $\check{R}$-matrix.

The same construction yields the $\mathbb{Z}_{+}^{m}$-graded $\mathrm{U}_{q}(\mathfrak{g})$-module algebra

$$
\mathcal{T}_{m}(V):=\left(T(V)^{\otimes m}, \mu_{T}\right)
$$

The next statement is an immediate consequence of Proposition 2.7 .

Lemma 2.9. The natural map $\tau^{\otimes m}: \mathcal{T}_{m} \longrightarrow \mathcal{A}_{m}$ is a surjection of $\mathbb{Z}_{+}^{m}$-graded $\mathrm{U}_{q}(\mathfrak{g})$-algebras.

It is useful to describe the map $\tau^{\otimes m}$ explicitly. For $\mathbf{a}^{(i)}=\left(a_{1}^{(i)}, \ldots, a_{d_{i}}^{(i)}\right)(i \in[1, m])$, let $t\left[\mathbf{a}^{(i)}\right]=v_{a_{1}^{(i)}} \otimes \cdots \otimes v_{a_{d_{i}}^{(i)}} \in T(V)_{d_{i}}$. Write $X\left[\mathbf{a}^{(i)}\right]=X_{i a_{1}^{(i)}} \cdots X_{i a_{d_{i}}^{(i)}} \in S_{q}(V)_{d_{i}}$. Then $\tau^{\otimes m}\left(t\left[\mathbf{a}^{(1)}\right] \cdots t\left[\mathbf{a}^{(m)}\right]\right)=X\left[\mathbf{a}^{(1)}\right] \cdots X\left[\mathbf{a}^{(m)}\right]$, where the right hand side belongs to the homogeneous component of $\mathcal{A}_{m}$ of multi-degree $\left(d_{1}, d_{2}, \ldots, d_{m}\right)$.

2.4. The quantum trace and invariants. It will be useful to keep in mind the structural context of the computations which appear below.

Denote $\mathrm{U}_{q}(\mathfrak{g})$ by $\mathrm{U}_{q}$. For any two $\mathrm{U}_{q}$-modules $N, M$, we have a canonical $\mathrm{U}_{q^{-}}$ module isomorphism $\xi: N \otimes M^{*} \stackrel{\sim}{\longrightarrow} \operatorname{Hom}_{\mathcal{K}}(M, N)$, where $\xi(n \otimes \phi): m \mapsto\langle\phi, m\rangle n$, and the action of $u \in \mathrm{U}_{q}$ on the right side is given by $u \cdot f(m)=\sum_{(u)} u_{(1)} \cdot f\left(S\left(u_{(2)}\right) m\right)$ for $f \in \operatorname{Hom}_{\mathcal{K}}(M, N)$, where $\Delta(u)=\sum_{(u)} u_{(1)} \otimes u_{(2)}$. The map $\xi$ induces an isomorphism

$$
\left(N \otimes M^{*}\right)^{\mathrm{U}_{q}} \stackrel{\sim}{\longrightarrow} \operatorname{Hom}_{\mathcal{K}}(M, N)^{\mathrm{U}_{q}}=\operatorname{Hom}_{\mathrm{U}_{q}}(M, N) .
$$

Now taking $M=V^{*}$ and $N=V$ in (2.10), we obtain an isomorphism $\xi_{0}$ : $\left(V \otimes V^{* *}\right)^{\mathrm{U}_{q}} \stackrel{\sim}{\longrightarrow} \operatorname{Hom}_{\mathrm{U}_{q}}\left(V^{*}, V\right)$. Write $K=K_{2 \rho}$, where $2 \rho$ is the sum of the positive roots (in the notation of [15]). There is an isomorphism of $\mathrm{U}_{q^{-}}$modules $\varepsilon_{K}: V \stackrel{\sim}{\longrightarrow} V^{* *}$ where $\varepsilon_{K}(v)=\varepsilon(K v)$, and $\varepsilon(w)$ is evaluation at $w \in V$. Composing these maps, we obtain an isomorphism

$$
f:(V \otimes V)^{\mathrm{U}_{q}} \longrightarrow\left(V \otimes V^{* *}\right)^{\mathrm{U}_{q}} \longrightarrow \operatorname{Hom}_{\mathrm{U}_{q}}\left(V^{*}, V\right) .
$$

If $V$ is simple, the dimension of the right side of (2.11) is 0 or 1 ; in this case any non-zero element $T \in(V \otimes V)^{\mathrm{U}_{q}}$ thus gives rise to an isomorphism of $\mathrm{U}_{q}$-modules $f_{T}: V^{*} \rightarrow V$.

Next, note that the map $\tau_{1}: V^{*} \otimes V \rightarrow \mathcal{K}$ given by $\phi \otimes v \mapsto\langle\phi, v\rangle$ is a homomorphism of $\mathrm{U}_{q}$-modules. Applying this with $V$ replaced by $V^{*}$, we see that the quantum trace $\tau_{q}: \operatorname{End}(V) \cong V \otimes V^{*} \rightarrow \mathcal{K}$ defined by $\tau_{q}(\xi(v \otimes \phi))=\langle\phi, K v\rangle$ is also a $\mathrm{U}_{q}$-module map. 
Now given two $\mathrm{U}_{q}$-modules $M, N$, we have an isomorphism of $\mathrm{U}_{q^{-}}$-modules $D$ : $M^{*} \otimes N^{*} \stackrel{\sim}{\longrightarrow}(M \otimes N)^{*}$, defined by $D=\kappa \circ R$, where $R$ is the $R$-matrix, and $\kappa$ is defined by $\langle\kappa(\phi \otimes \psi), m \otimes n\rangle=\langle\phi, m\rangle\langle\psi, n\rangle$. This is because for $u \in \mathrm{U}_{q}$, $\Delta \circ S(u)=S \otimes S \circ \Delta^{\prime}(u)$, where $\Delta^{\prime}$ is the opposite comultiplication to $\Delta$.

It follows that $\tau_{1} \in\left(\left(V^{*} \otimes V\right)^{*}\right)^{\mathrm{U}_{q}} \simeq\left(V \otimes V^{*}\right)^{\mathrm{U}_{q}}$ and similarly that $\tau_{q} \in((V \otimes$ $\left.\left.V^{*}\right)^{*}\right)^{\mathrm{U}_{q}} \simeq\left(V^{*} \otimes V\right)^{\mathrm{U}_{q}}$

\section{Invariants in terms of basis elements.}

We shall make explicit the above identifications in terms of bases of $V$ and $V^{*}$.

Let $V$ be a $\mathrm{U}_{q}$-module, and let $v_{1}, \ldots, v_{n}$ be a basis of weight vectors, with wt $\left(v_{i}\right)=$ $\lambda_{i}$; we assume the $\lambda_{i}$ are pairwise distinct. Let $v_{1}^{*}, \ldots, v_{n}^{*}$ be the dual basis of $V^{*}$; then $\operatorname{wt}\left(v_{i}^{*}\right)=-\lambda_{i}$. Under the isomorphism (2.10), $\mathrm{id}_{V}$ corresponds to $\gamma:=\sum_{i} v_{i} \otimes v_{i}^{*} \in$ $V \otimes V^{*}$. Since the former is $\mathrm{U}_{q}$-invariant, we have

$$
\gamma=\sum_{i} v_{i} \otimes v_{i}^{*} \in\left(V \otimes V^{*}\right)^{\mathrm{U}_{q}}
$$

Now the map $\epsilon_{K}: V \rightarrow V^{* *}$ takes $v_{r}$ to $q^{\left(2 \rho, \lambda_{r}\right)} v_{r}^{* *}$ where $\left(v_{r}^{* *}\right)$ is the basis of $V^{* *}$ dual to the basis $\left(v_{r}^{*}\right)$ of $V^{*}$. Applying $\epsilon_{K} \otimes \operatorname{id}_{V^{*}}$ to $\gamma$ and reinterpreting in terms of $V$ (rather than $V^{*}$ ), we see (taking into account that $v_{r}^{*}$ has weight $-\lambda_{r}$ ) that

$$
\gamma_{q}=\sum_{i} q^{-\left(2 \rho, \lambda_{i}\right)} v_{i}^{*} \otimes v_{i} \in\left(V^{*} \otimes V\right)^{\mathrm{U}_{q}} .
$$

If $V$ is simple then $\left(V \otimes V^{*}\right)^{\mathrm{U}_{q}} \simeq\left(V^{*} \otimes V\right)^{\mathrm{U}_{q}}$ is one-dimensional. Hence the element $\gamma_{q}$ of (2.13) is a non-zero scalar multiple of the quantum trace $\tau_{q}$.

Now assume that $V$ is simple and self dual. Then whenever $\lambda_{r}$ is a weight of $V$, so is $-\lambda_{r}$, and we write $v_{-r}$ for the basis element with weight $-\lambda_{r}$. Since any element $T \in(V \otimes V)^{\mathrm{U}_{q}}$ has weight zero, it must be of the form

$$
T=\sum_{r=1}^{n} c_{r} v_{r} \otimes v_{-r},
$$

for elements $c_{r} \in \mathcal{K}$. The corresponding $\mathrm{U}_{q}$-isomorphism $f_{T}: V^{*} \rightarrow V$ (2.11) is readily seen to satisfy

$$
f_{T}\left(v_{i}^{*}\right)=c_{-i} q^{\left(2 \rho, \lambda_{i}\right)} v_{-i} .
$$

Since $f_{T}$ is an isomorphism, it follows that $c_{i} \neq 0$ for all $i$, and the inverse $f_{T}^{-1}$ : $V \rightarrow V^{*}$ is described similarly.

We may now apply $\mathrm{id}_{V} \otimes f_{T}^{-1}$ and $f_{T}^{-1} \otimes \mathrm{id}_{V}$ to $T$, to obtain invariant elements of $V \otimes V^{*}$ and $V^{*} \otimes V$ respectively. The latter yields the element $\gamma_{q}$ of (2.13), while the former yields $\sum_{i} c_{i} c_{-i}^{-1} q^{-\left(2 \rho, \lambda_{i}\right)} v_{i} \otimes v_{i}^{*}$. Comparing with $\gamma(\underline{2.13})$ shows that

$$
c_{i} c_{-i}^{-1} q^{-\left(2 \rho, \lambda_{i}\right)} \text { is independent of } i \text {. }
$$

It is evident that if zero is a weight of $V$, then the implied constant is 1 .

In the next three sections, we shall study the algebras $\mathcal{A}_{m}$ associated with the natural modules of the quantum orthogonal and symplectic groups. 


\section{INVARIANT THEORY OF THE QUANTUM EVEN ORTHOGONAL GROUPS $\mathrm{U}_{q}\left(\mathfrak{s o}_{2 n}\right)$}

Let $\epsilon_{i}(i=1,2, \ldots, n)$ be an orthonormal basis of the weight lattice of the Lie algebra $\mathfrak{s o}_{2 n}$; a set of simple roots may then be taken to be $\epsilon_{i}-\epsilon_{i+1} 1 \leq i<n$ together with $\epsilon_{n-1}+\epsilon_{n}$. In this section, $\mathrm{U}_{q}$ will denote the quantum group $\mathrm{U}_{q}\left(\mathfrak{s o}_{2 n}\right)$.

3.1. The natural module for $\mathrm{U}_{q}\left(\mathfrak{s o}_{2 n}\right)$. We realise the group $S O_{2 n}(\mathbb{C})$ as the subgroup of $S L_{2 n}(\mathbb{C})$ preserving the bilinear form defined by the matrix $J:=\left[\begin{array}{cc}0 & I_{n} \\ I_{n} & 0\end{array}\right]$, where $I_{n}$ is the $n \times n$ identity matrix. Then $\mathfrak{g}=\mathfrak{s o}_{2 n}$ is the subalgebra of $\mathfrak{s l}_{2 n}(\mathbb{C})$ consisting of matrices satisfying $X^{t}=-J X J$. Accordingly, there is a Cartan subalgebra consisting of diagonal matrices. Now the natural representation of $\mathfrak{s o}_{2 n}$ is minuscule. Thus it lifts to the natural representation of the quantum group $\mathrm{U}_{q}$ in such a way that matrices for the Chevalley generators remain the same (see, e.g. [31]); this is exploited in the description below. The natural module $V$ for $\mathrm{U}_{q}$ has highest weight $\epsilon_{1}$ and weights $\pm \epsilon_{i}, i=1,2, \ldots, n$. It therefore has a basis $\left\{v_{a} \mid a \in[1, n] \cup[-n,-1]\right\}$, where $[1, n]=\{1,2, \ldots, n\}$ and $[-n,-1]=-n,-(n-1), \ldots,-1$, and $v_{a}$ has weight $\operatorname{sgn}(a) \epsilon_{|a|}$, where $\operatorname{sgn}(a)=a /|a|$. Let $E_{a b}$ be the matrix units in $\operatorname{End}(V)$ relative to this basis, defined by

$$
E_{a b} v_{c}=\delta_{b c} v_{a} .
$$

Then we have the following explicit formulae for the natural representation $\pi$ : $\mathrm{U}_{q} \longrightarrow \operatorname{End}(V)$ of $\mathrm{U}_{q}$ relative to the above basis:

$$
\begin{aligned}
& \pi\left(e_{i}\right)=E_{i, i+1}-E_{-i-1,-i}, \quad \pi\left(f_{i}\right)=E_{i+1, i}-E_{-i,-i-1}, \quad i<n, \\
& \pi\left(e_{n}\right)=E_{n-1,-n}-E_{n,-n+1}, \quad \pi\left(f_{n}\right)=E_{-n, n-1}-E_{-n+1, n}, \\
& \pi\left(k_{i}\right)=1+(q-1)\left(E_{i i}+E_{-i-1,-i-1}\right)+\left(q^{-1}-1\right)\left(E_{i+1, i+1}+E_{-i,-i}\right), \quad i<n, \\
& \pi\left(k_{n}\right)=1+(q-1)\left(E_{n-1, n-1}+E_{n n}\right)+\left(q^{-1}-1\right)\left(E_{-n+1,-n+1}+E_{-n,-n}\right) .
\end{aligned}
$$

Note that the subalgebra of $\mathrm{U}_{q}$ generated by $e_{i}, f_{i}, k_{i}^{ \pm 1}(i<n)$ is isomorphic to $\mathrm{U}_{q}\left(\mathfrak{s l}_{n}\right)$, and the vectors $v_{i}(i \leq n)$ span its natural module. The $v_{-i}(i \leq n)$ span its dual.

The tensor product $V \otimes V$ is the direct sum of three distinct irreducible submodules $L_{0}, L_{2 \epsilon_{1}}$ and $L_{\epsilon_{1}+\epsilon_{2}}$ with highest weights $0,2 \epsilon_{1}$ and $\epsilon_{1}+\epsilon_{2}$ respectively. The following explicit bases for these irreducible summands will be useful later.

(1) A basis for $L_{0}$ (note that this is an element of the form of $T$ in (2.14) ):

$$
\sum_{i=1}^{n}\left(q^{n-i} v_{i} \otimes v_{-i}+q^{i-n} v_{-i} \otimes v_{i}\right) .
$$

(2) A basis for $L_{2 \epsilon_{1}}$ :

$$
\begin{aligned}
& v_{i} \otimes v_{i}, \quad v_{-i} \otimes v_{-i}, \quad 1 \leq i \leq n, \\
& v_{i} \otimes v_{j}+q v_{j} \otimes v_{i}, \quad v_{-j} \otimes v_{-i}+q v_{-i} \otimes v_{-j}, \quad i<j \leq n, \\
& v_{i} \otimes v_{-j}+q v_{-j} \otimes v_{i}, \quad i \neq j, \\
& q^{-1} v_{i} \otimes v_{-i}+q v_{-i} \otimes v_{i}-\left(v_{i+1} \otimes v_{-i-1}+v_{-i-1} \otimes v_{i+1}\right), \quad i \leq n-1 .
\end{aligned}
$$


(3) A basis for $L_{\epsilon_{1}+\epsilon_{2}}$ :

$$
\begin{array}{ll} 
& v_{i} \otimes v_{j}-q^{-1} v_{j} \otimes v_{i}, \quad v_{-j} \otimes v_{-i}-q^{-1} v_{-i} \otimes v_{-j}, \quad i<j \leq n, \\
& v_{i} \otimes v_{-j}-q^{-1} v_{-j} \otimes v_{i}, \quad i \neq j, \\
(3.4) & v_{i} \otimes v_{-i}-v_{-i} \otimes v_{i}-\left(q v_{i+1} \otimes v_{-i-1}-q^{-1} v_{-i-1} \otimes v_{i+1}\right), \quad i<n-1, \\
& v_{n-1} \otimes v_{1-n}-v_{1-n} \otimes v_{n-1}-\left(q v_{n} \otimes v_{-n}-q^{-1} v_{-n} \otimes v_{n}\right), \\
& v_{n-1} \otimes v_{1-n}-v_{1-n} \otimes v_{n-1}+\left(q^{-1} v_{n} \otimes v_{-n}-q v_{-n} \otimes v_{n}\right) .
\end{array}
$$

Let $P_{s}, P_{a}$ and $P_{0}$ be the idempotent projections mapping $V \otimes V$ on to the irreducible submodules with highest weights $2 \epsilon_{1}, \epsilon_{1}+\epsilon_{2}$ and 0 respectively. Then the $R$-matrix of $\mathrm{U}_{q}$ acting on $V \otimes V$ is given by

$$
\check{R}=q P_{s}-q^{-1} P_{a}+q^{1-2 n} P_{0} .
$$

In Section 3.3 , we shall need the $R$-matrix in the following slightly more general situation for the proof of Lemma 3.6. Let $V_{1}$ and $V_{2}$ be two isomorphic copies of the natural module. Denote by $P_{\mu}^{(1,2)}$ the idempotent projection from $V_{1} \otimes V_{2}$ onto its irreducible submodule with highest weight $\mu$, where $\mu=2 \epsilon_{1}, \epsilon_{1}+\epsilon_{2}$ or 0 . Similarly, define the idempotents $P_{\mu}^{(2,1)}$ in $\operatorname{End}\left(V_{2} \otimes V_{1}\right)$. Then the $R$-matrix

$$
\check{R}: V_{1} \otimes V_{2} \longrightarrow V_{2} \otimes V_{1}
$$

is the (unique) $\mathrm{U}_{q}$-linear map satisfying the following relations:

$$
\check{R} P_{2 \epsilon_{1}}^{(1,2)}=q P_{2 \epsilon_{1}}^{(2,1)}, \quad \check{R} P_{\epsilon_{1}+\epsilon_{1}}^{(1,2)}=-q^{-1} P_{\epsilon_{1}+\epsilon_{2}}^{(2,1)}, \quad \check{R} P_{0}^{(1,2)}=q^{1-2 n} P_{0}^{(2,1)} .
$$

In particular, if we denote by $\left\{v_{b}^{(\alpha)} \mid b \in[-n,-1] \cup[1, n]\right\}$ the standard basis for $V_{\alpha}$ $(\alpha=1,2)$, and let $T^{(\alpha, \beta)}=\sum_{i=1}^{n}\left(q^{n-i} v_{i}^{(\alpha)} \otimes v_{-i}^{(\beta)}+q^{i-n} v_{-i}^{(\alpha)} \otimes v_{i}^{(\beta)}\right)$, we have

$$
\check{R} T^{(1,2)}=q^{1-2 n} T^{(2,1)} \text {. }
$$

3.2. The braided symmetric algebra of the natural $\mathrm{U}_{q}\left(\mathfrak{s o}_{2 n}\right)$-module. Let $T(V)$ be the tensor algebra of the natural $\mathrm{U}_{q}$-module $V$. Then $T(V)=\bigoplus_{k=0}^{\infty} V^{\otimes k}$. The submodule $P_{a}(V \otimes V) \cong L_{\epsilon_{1}+\epsilon_{2}}$ generates a graded two-sided ideal $\mathcal{I}_{q}$ of $T(V)$ which is also graded since it is generated by homogeneous elements. Hence the braided symmetric algebra $S_{q}(V)=T(V) / \mathcal{I}_{q}$ of $V$ inherits a $\mathbb{Z}_{+}$-grading from $T(V)$. It is evident, given the basis of $L_{\epsilon_{1}+\epsilon_{2}}$ constructed above, that the following is a presentation of $S_{q}(V)$. Here we abuse notation by writing $v_{j}$ for the image in $S_{q}(V)$ of $v_{j} \in T(V)$.

Lemma 3.1. The braided symmetric algebra $S_{q}(V)$ of the natural $\mathrm{U}_{q}\left(\mathfrak{s o}_{2 n}\right)$-module $V$ is generated by $\left\{v_{a} \mid a \in[-n,-1] \cup[1, n]\right\}$ subject to the following relations

$$
\begin{aligned}
& v_{i} v_{j}-q^{-1} v_{j} v_{i}=0, \quad 1 \leq i<j \leq n, \\
& v_{-j} v_{-i}-q^{-1} v_{-i} v_{-j}=0, \quad 1 \leq i<j \leq n, \\
& v_{i} v_{-j}-q^{-1} v_{-j} v_{i}=0, \quad i \neq j, \\
& v_{i} v_{-i}-v_{-i} v_{i}=q v_{i+1} v_{-i-1}-q^{-1} v_{-i-1} v_{i+1}, \quad 1 \leq i \leq n-1, \\
& v_{n} v_{-n}-v_{-n} v_{n}=0 .
\end{aligned}
$$


Note that the last two sets of relations may be written as

$$
v_{-i} v_{i}=v_{i} v_{-i}-\left(q-q^{-1}\right) q^{i+1-n} \phi_{i+1}^{(+)}, \quad i \in[1, n],
$$

where $\phi_{i}^{(+)}$is the quadratic element $\sum_{k=i}^{n} q^{n-k} v_{k} v_{-k}$. Set $\phi_{i}^{(-)}=\sum_{k=i}^{n} q^{k-n} v_{-k} v_{k}$. An easy computation using (3.10) shows that $\phi_{i}^{(+)}=q^{2 n-2} \phi_{i}^{(-)}$for all $i$.

Using results of [34] or by direct calculation, one sees that the ordered monomials in $v_{i}$ and $v_{-i}(i \in[1, n])$ form a basis of $S_{q}(V)$. That is, $S_{q}(V)$ is a flat deformation of the symmetric algebra of $V$ in the sense of [3].

In summary, we have

Theorem 3.2. (1) The braided symmetric algebra $S_{q}(V)$ of $V$ is a $\mathbb{Z}_{+}$-graded module algebra over $\mathrm{U}_{q}\left(\mathfrak{s o}_{2 n}\right)$.

(2) The ordered monomials in $v_{i}, v_{-i}(i \in[1, n])$ form a basis of $S_{q}(V)$.

Let $\Phi:=\phi_{1}^{(+)}+\phi_{1}^{(-)}$. Then

$$
\Phi=q^{1-n}\left(q^{n-1}+q^{1-n}\right) \phi_{1}^{(+)} .
$$

Proposition 3.3. (1) We have $\Phi \in S_{q}(V)^{\mathrm{U}_{q}\left(\mathfrak{s o}_{2 n}\right)}$.

(2) The element $\Phi$ belongs to the centre of $S_{q}(V)$.

Proof. The first statement is immediate because $\Phi$ is the image in $S_{q}(V)$ of the basis element in (3.2) of $L_{0}:=(V \otimes V)^{\mathrm{U}_{q}}$.

In view of (3.11), to prove (2), it clearly suffices to show that $\phi_{1}^{(+)}$is central. Consider $v_{j} \phi_{1}^{(+)}$: we have

$$
\begin{aligned}
v_{j} \phi_{1}^{(+)} & =\sum_{i=1}^{j-1} q^{n-i} v_{i} v_{-i} v_{j}+q^{n-j} v_{j} v_{j} v_{-j}+q^{-2} \phi_{j+1}^{(+)} v_{j} \\
& =\phi_{1}^{(+)} v_{j}+\left(q^{-2}-1\right) \phi_{j+1}^{(+)} v_{j}+q^{n-j} v_{j}\left(v_{j} v_{-j}-v_{-j} v_{j}\right) .
\end{aligned}
$$

By (3.10), we have

$$
q^{n-j} v_{j}\left(v_{j} v_{-j}-v_{-j} v_{j}\right)=\left(q^{2}-1\right) v_{j} \phi_{j+1}^{(+)}=-\left(q^{-2}-1\right) \phi_{j+1}^{(+)} v_{j} .
$$

It follows that $v_{j}$ commutes with $\phi_{1}^{(+)}$for all $j \geq 0$. Similarly one shows that $v_{-j}$ commutes with $\phi_{1}^{(+)}$for all $j$, and the proposition follows.

Let $S_{q}(V)^{\mathrm{U}_{q}\left(\mathfrak{s o}_{2 n}\right)}$ be the space of $\mathrm{U}_{q}$-invariants in $S_{q}(V)$; these form a subalgebra by Lemma 2.2 .

Proposition 3.4. The subalgebra $S_{q}(V)^{\mathrm{U}_{q}\left(\mathfrak{s o}_{2 n}\right)}$ of invariants is generated by $\Phi$ and is isomorphic to the polynomial algebra $\mathbb{C}[\Phi]$.

Proof. Denote by $S_{q}(V)_{k}$ the homogeneous subspace of degree $k$ in $S_{q}(V)$. Let $S_{q}^{\prime}(V)_{k}$ be the $\mathrm{U}_{q}$-submodule of $S_{q}(V)_{k}$ generated by $\left(v_{1}\right)^{k}$, that is $S_{q}^{\prime}(V)_{k}=\mathrm{U}_{q} \cdot\left(v_{1}\right)^{k}$. Then $S_{q}^{\prime}(V)_{k}$ is isomorphic to the irreducible $\mathrm{U}_{q}$-module with highest weight $k \epsilon_{1}$, and thus has dimension $\left[\begin{array}{c}2 n+k-1 \\ k\end{array}\right]-\left[\begin{array}{c}2 n+k-3 \\ k-2\end{array}\right]$. Taking into account the weights of $\mathrm{U}_{q}$ occurring in $S_{q}(V)_{k-2}$, one sees that $S_{q}^{\prime}(V)_{k} \cap \Phi S_{q}(V)_{k-2}=0$. Now 
$\operatorname{dim} S_{q}(V)_{k}=\left[\begin{array}{c}2 n+k-1 \\ k\end{array}\right]=\operatorname{dim} S_{q}^{\prime}(V)_{k}+\operatorname{dim} S_{q}(V)_{k-2}$, and recalling that $\Phi$ is invariant, we have the $\mathrm{U}_{q}$-module decomposition $S_{q}(V)_{k}=S_{q}^{\prime}(V)_{k} \oplus \Phi S_{q}(V)_{k-2}$.

It follows that $S_{q}(V)_{k}^{\mathrm{U}_{q}}=S_{q}^{\prime}(V)_{k}^{\mathrm{U}_{q}} \oplus\left(\Phi S_{q}(V)_{k-2}\right)^{\mathrm{U}_{q}}$. Since $S_{q}^{\prime}(V)_{k}$ is a nontrivial irreducible $\mathrm{U}_{q}$-module for all $k>0, S_{q}^{\prime}(V)_{k}^{\mathrm{U}_{q}}=0$, and since $\Phi$ is invariant,

$$
\left(S_{q}(V)_{k}\right)^{\mathrm{U}_{q}}=\Phi\left(S_{q}(V)_{k-2}\right)^{\mathrm{U}_{q}} .
$$

It follows that $\left(S_{q}(V)_{k}\right)^{\mathrm{U}_{q}\left(\mathfrak{s o}_{2 n}\right)}$ is spanned by $\Phi^{\frac{k}{2}}$ for even $k$ and is 0 for odd $k$.

The results of this section may be applied to the construction of a quantum sphere $\mathbb{S}_{q}^{2 n-1}$ with manifest quantum orthogonal group symmetry, that is, with an action of $\mathrm{U}_{q}\left(\mathfrak{s o}_{2 n}\right)$. Since $\Phi$ is a central element in $S_{q}(V)$ by Proposition 3.3 (2), the left ideal generated by $\Phi-1$ coincides with the right, and hence is a two-sided ideal, which we denote by $\langle\Phi-1\rangle$. Then the quantum sphere $\mathbb{S}_{q}^{2 n-1}$ is defined by

$$
\mathbb{S}_{q}^{2 n-1}=S_{q}(V) /\langle\Phi-1\rangle .
$$

From the proof of Proposition 3.4, we have the following result, analogous to its classical $(q=1)$ counterpart.

Lemma 3.5. The quantum sphere $\mathbb{S}_{q}^{2 n-1}$ is a $\mathrm{U}_{q}\left(\mathfrak{s o}_{2 n}\right)$-module algebra whose decomposition as $\mathrm{U}_{q}\left(\mathfrak{s o}_{2 n}\right)$-module is $\mathbb{S}_{q}^{2 n-1}=\bigoplus_{k=0}^{\infty} L_{k \epsilon_{1}}$.

3.3. A $\mathrm{U}_{q}\left(\mathfrak{s o}_{2 n}\right)$ module algebra: $S_{q}(V)^{\otimes m}$ with twisted multiplication. Let $\mathcal{T}_{m}=\left(T(V)^{\otimes m}, \mu_{T}\right)$ be the $\mathrm{U}_{q}$-module algebra with $\mathrm{U}_{q}=\mathrm{U}_{q}\left(\mathfrak{s o}_{2 n}\right)$ where multiplication $\mu_{T}$ is defined by iterating (2.4). We shall construct the quantum analogue of the coordinate ring of $\oplus^{m} V$ as a module algebra $\mathcal{A}_{m}=\left(S_{q}(V)^{\otimes m}, \mu_{S}\right)$ over $\mathrm{U}_{q}$ by repeatedly using (2.4).

It will be convenient to relabel the standard basis elements of $V$ as $v_{a}$ with $a \in$ $[1,2 n]$, where $v_{a}$ is identified with $v_{a-2 n-1}$ if $a>n$. The following result describes the algebraic structure of $\mathcal{A}_{m}$. For $i \in[1, m]$ and $a \in[1,2 n]$, let $X_{i a}$ be the image in $\mathcal{A}_{m}$ of $1 \otimes \cdots \otimes 1 \otimes v_{a} \otimes 1 \otimes \cdots \otimes 1$, where $v_{a}$ is the $i^{\text {th }}$ factor.

Lemma 3.6. The $\mathrm{U}_{q}\left(\mathfrak{s o}_{2 n}\right)$-module algebra $\mathcal{A}_{m}$ is generated by $X_{i a}$ with $i \in[1, m]$ and $a \in[1,2 n]$, subject to the following relations:

(1) for fixed $i$, the elements $X_{i a}$ obey the relations (3.9) with $v_{a}=X_{i a}$;

(2) for $i<j$ in $[1, m]$ and $a, b \in[1,2 n]$ :

$$
\begin{aligned}
& X_{j a} X_{i a}=q X_{i a} X_{j a}, \quad \forall a, \\
& X_{j b} X_{i a}=X_{i a} X_{j b}+\left(q-q^{-1}\right) X_{i b} X_{j a}, \quad a<b \neq 2 n+1-a, \\
& X_{j a} X_{i b}=X_{i b} X_{j a}, \quad a<b \neq 2 n+1-a, \\
& X_{j t} X_{i, 2 n+1-t}=q X_{i, 2 n+1-t} X_{j t}-\left(q-q^{-1}\right) q^{n-t} \psi_{t}^{(i, j)}, \\
& X_{j, 2 n+1-t} X_{i t}=q X_{i t} X_{j, 2 n+1-t}-\left(q-q^{-1}\right) X_{i, 2 n+1-t} X_{j t} \\
& +\left(q-q^{-1}\right) q^{t-n}\left(\bar{\psi}_{t+1}^{(i, j)}-\Psi^{(i, j)}\right), \quad t \in[1, n],
\end{aligned}
$$


where (for all $i, j \in[1, m]$ )

$$
\begin{aligned}
\psi_{t}^{(i, j)} & =\sum_{k=1}^{t} q^{k-n} X_{i, 2 n+1-k} X_{j k}, \\
\bar{\psi}_{t}^{(i, j)} & =\sum_{k=1}^{t} q^{n-k} X_{i k} X_{j, 2 n+1-k}, \\
\Psi^{(i, j)} & =\psi_{n}^{(i, j)}+\bar{\psi}_{n}^{(i, j)} .
\end{aligned}
$$

Proof. For fixed $i$, the elements $X_{i a}(i \in[1,2 n])$ generate a subalgebra isomorphic to $S_{q}(V)$ in $\mathcal{A}_{m}$, whence (1).

Part (2) is obtained from (2.8) by straightforward but tedious calculation. We indicate the main steps. First, using (3.7), we rewrite relation (2.8) for the present case in the following form (for all $i<j$ ):

$$
\begin{aligned}
& \quad X_{j a} X_{i a}=q X_{i a} X_{j a}, \forall a, \\
& \quad X_{j a} X_{i b}+q X_{j b} X_{i a} \\
& =q\left(X_{i a} X_{j b}+q X_{i b} X_{j a}\right), \quad a<b \neq 2 n+1-a, \\
& \quad q^{-1} X_{j t} X_{i, 2 n+1-t}+q X_{j, 2 n+1-t} X_{i t} \\
& \quad-\left(X_{j, t+1} X_{i, 2 n-t}+X_{j, 2 n-t} X_{i, t}\right) \\
& \quad=q\left(q^{-1} X_{i t} X_{j, 2 n+1-t}+q X_{i, 2 n+1-t} X_{j t}\right) \\
& \quad-q\left(X_{i, t+1} X_{j, 2 n-t}+X_{i, 2 n-t} X_{j, t}\right), \quad 1 \leq t<n ; \\
& X_{j a} X_{i b}-q^{-1} X_{j b} X_{i a} \\
& =-q^{-1}\left(X_{i a} X_{j b}-q^{-1} X_{i b} X_{j a}\right), \quad a<b \neq 2 n+1-a, \\
& X_{j t} X_{i, 2 n+1-t}-X_{j, 2 n+1-t} X_{i t} \\
& -\left(q X_{j, t+1} X_{i, 2 n-t}-q^{-1} X_{j, 2 n-t} X_{i, t}\right) \\
& =-q^{-1}\left(X_{i t} X_{j, 2 n+1-t}-X_{i, 2 n+1-t} X_{j t}\right) \\
& +q^{-1}\left(q X_{i, t+1} X_{j, 2 n-t}-q^{-1} X_{i, 2 n-t} X_{j, t}\right), \quad 1 \leq t<n, \\
& X_{j n} X_{i, n+1}-X_{j, n+1} X_{i n} \\
& =-q^{-1}\left(X_{i n} X_{j, n+1}-X_{i, n+1} X_{j n}\right) ; \\
& \quad \Psi^{(j, i)}=q^{1-2 n} \Psi^{(i, j)} .
\end{aligned}
$$

The first relation of (3.13) is just the first relation of (3.15), and the other relations of (3.13) are obtained by combining the second relation of (3.15) with the first relation in (3.16) (that is, the relations with $a<b \neq 2 n+1-a)$.

The relations (3.14) are obtained from the third relation of (3.15), the second and third relations of (3.16) and the relation (3.17) by a rather lengthy sequence of routine manipulations.

The fact that these relations suffice is a consequence of the flat nature of $S_{q}(V)$, which implies that there is a linear isomorphism $\mathcal{A}_{m} \longrightarrow S(V)^{\otimes m}$.

The elements $\Psi^{(i, j)}$ will play an important role in the study of the subalgebra of $\mathrm{U}_{q}$-invariants in $\mathcal{A}_{m}$, and we now study their properties. 
Lemma 3.7. (1) For all $i, j \in[1, m]$, the elements $\Psi^{(i, j)}$ are $\mathrm{U}_{q^{-} \text {invariant. }}$

(2) The elements $\Psi^{(i, j)}$ satisfy (3.17) as well as the following relations:

$$
\begin{aligned}
& X_{k a} \Psi^{(i, i)}-\Psi^{(i, i)} X_{k a}=0, \quad \text { for all } i, k, \\
& X_{k a} \Psi^{(i, j)}-\Psi^{(i, j)} X_{k a}=0, \quad k>i, j \quad \text { or } k<i, j, \\
& X_{k a} \Psi^{(i, j)}-\Psi^{(i, j)} X_{k a} \\
& =\left(q-q^{-1}\right)\left(X_{i a} \Psi^{(k, j)}-\Psi^{(i, k)} X_{j a}\right), \quad i<k<j, \\
& \Psi^{(i, j)} X_{i a}-q^{-1} X_{i a} \Psi^{(i, j)}=\left(q-q^{-1}\right) \bar{\psi}_{n}^{(i, i)} X_{j a}, \quad i<j, \\
& X_{j a} \Psi^{(i, j)}-q^{-1} \Psi^{(i, j)} X_{j a}=\left(q-q^{-1}\right) X_{i a} \bar{\psi}_{n}^{(j, j)}, \quad i<j .
\end{aligned}
$$

Proof. Observe that the image of $V$ under the natural map $T(V) \rightarrow S_{q}(V)$ is a copy of $V$ in $S(V)$. Hence for $i<j, \Psi^{(i, j)}$ may be thought of as the basis element (3.2) of $(V \otimes V)^{\mathrm{U}_{q}}$ where the two copies of $V$ are in the $i^{\text {th }}$ and $j^{\text {th }}$ factors of $\otimes^{m} S_{q}(V)$. Taking Proposition 3.3 into account, it follows that $\Psi^{(i, j)}$ is invariant if $i \leq j$. By equation (3.17), (1) follows.

The $k=i$ case of the first relation of (3.18) follows from Proposition 3.3. Write the universal $R$-matrix of $\mathrm{U}_{q}$ as $R=\sum_{t} \alpha_{t} \otimes \beta_{t}$. If $k>i, j$, we have

$$
X_{k a} \Psi^{(i, j)}=\sum_{t} \beta_{t}\left(\Psi^{(i, j)}\right) \alpha_{t}\left(X_{k a}\right) .
$$

Since $\Psi^{(i, j)}$ is $\mathrm{U}_{q}$-invariant by part (1), the right side is equal to

$$
\sum_{t} \epsilon\left(\beta_{t}\right)\left(\Psi^{(i, j)}\right) \alpha_{t}\left(X_{k a}\right)=\Psi^{(i, j)} X_{k a},
$$

proving the second relation of (3.18) for $k>i, j$. The case $k<i, j$ is similar. Taking $i=j$, we obtain the case $k \neq i$ of the first relation of (3.18).

To prove the third relation of (3.18), it suffices to consider the case $i=1, k=2$ and $j=3$. Since $S_{q}(V)_{1} \cong T(V)_{1} \cong V$, we have canonical $\mathrm{U}_{q}$-module isomorphisms

$$
V \otimes V \otimes V \longrightarrow\left(\mathcal{T}_{3}(V)\right)_{(1,1,1)} \longrightarrow\left(\mathcal{A}_{3}\right)_{(1,1,1)},
$$

where the second map is the restriction of $\tau^{\otimes 3}$ to $\left(\mathcal{T}_{3}(V)\right)_{(1,1,1)}$. Denote the first map by $\iota_{(1,1,1)}$. Our strategy is to deduce the third relation of (3.18) from appropriate relations in $V \otimes V \otimes V$.

This will be done using the diagrammatical method of Reshetikhin-Turaev (see [25, 26]), which is equivalent to working in the $B M W$-algebra, to describe homomorphisms between tensor powers of $V$. Recall that their functor sends tangle diagrams to $\mathrm{U}_{q}$-module homomorphisms. In our case, we shall colour all the components of any tangle diagram with the module $V$, so that the images of tangle diagrams under the Reshetikhin-Turaev functor are $\mathrm{U}_{q}$-maps between tensor powers of $V$. Because the module $V$ is self dual, there is no need to orient the tangle diagrams.

We shall identify tangle diagrams with the corresponding $\mathrm{U}_{q}$-module homomorphisms; the relations we seek will arise from relations among diagrams whose images lie in $V^{\otimes 3}$. Write the basis element (3.2) of $L_{0} \subset V \otimes V$ defined in Section 3.1 as $\sum_{a, b} C_{a b} v_{a} \otimes v_{b}$ with $C_{a b} \in \mathcal{K}$; then in terms of diagrams, we have $\bigvee: \mathcal{K} \longrightarrow V \otimes V$, 
$1 \mapsto \sum_{a, b} C_{a b} v_{a} \otimes v_{b}$. Furthermore, we have the following skein relation:

$$
\psi-\bigcup_{1}=\left(q-q^{-1}\right)(|V-V|) \text {. }
$$

Let us denote

$$
\begin{array}{cc}
D_{+}:=\bigcup: V \longrightarrow V^{\otimes 3}, & D_{-}:=\bigcup^{\prime}: V \longrightarrow V^{\otimes 3}, \\
D_{0}:=\|: V \longrightarrow V^{\otimes 3}, & D_{0}^{\prime}:=\bigcup^{\prime}: V \longrightarrow V^{\otimes 3} .
\end{array}
$$

Then

$$
\begin{array}{ll}
D_{0}\left(v_{a}\right)=v_{a} \otimes \sum_{b, d} C_{b d} v_{b} \otimes v_{d}, & D_{0}^{\prime}\left(v_{a}\right)=\sum_{b, d} C_{b d} v_{b} \otimes v_{d} \otimes v_{a}, \\
D_{+}\left(v_{a}\right)=\sum_{b, d} C_{b d} \check{R}\left(v_{a} \otimes v_{b}\right) \otimes v_{d}, & D_{-}\left(v_{a}\right)=\sum_{b, d} C_{b d} v_{b} \otimes \check{R}\left(v_{d} \otimes v_{a}\right),
\end{array}
$$

from which we obtain

$$
\begin{array}{rlrl}
\tau^{\otimes 3} \circ \iota_{(1,1,1)} \circ D_{+}\left(v_{a}\right) & =X_{2 a} \Psi^{(1,3)}, & & \tau^{\otimes 3} \circ \iota_{(1,1,1)} \circ D_{-}\left(v_{a}\right)=\Psi^{(1,3)} X_{2 a}, \\
\tau^{\otimes 3} \circ \iota_{(1,1,1)} \circ D_{0}\left(v_{a}\right)=X_{1 a} \Psi^{(2,3)}, & & \tau^{\otimes 3} \circ \iota_{(1,1,1)} \circ D_{0}^{\prime}\left(v_{a}\right)=\Psi^{(1,2)} X_{3 a} .
\end{array}
$$

Evaluating both sides of (3.19) at $v_{a}$, then applying the map $\tau^{\otimes 3} \circ \iota_{(1,1,1)}$ to the resulting elements of $V^{\otimes 3}$, we obtain the third relation of (3.18) for $i=1, k=2$ and $j=3$.

The fourth and fifth relations of (3.18) can be proved in much the same way, and we shall consider the fourth relation only. We may assume that $i=1$ and $j=2$. Note that the map $\tau^{\otimes 2}: \mathcal{T}_{2}(V) \longrightarrow \mathcal{A}_{2}$ restricts to an isomorphism $\left(P_{s}+\right.$ $\left.P_{0}\right) T(V)_{2} \otimes T(V)_{1} \cong S_{q}(V)_{2} \otimes S_{q}(V)_{1}$. Denote by $\iota_{(2,1)}$ the canonical $\mathrm{U}_{q}$-isomorphism from $(V \otimes V) \otimes V$ to $T(V)_{2} \otimes T(V)_{1}$. Let

$$
\begin{aligned}
& F:=\left(\left(P_{s}+P_{0}\right) \otimes \mathrm{id}\right) D_{0}=\left(P_{s} \otimes \mathrm{id}\right) D_{0}+\frac{1}{\operatorname{dim}_{q} V} D_{0}^{\prime}, \\
& B:=\left(\left(P_{s}+P_{0}\right) \otimes \mathrm{id}\right) D_{-}=q^{-1}\left(P_{s} \otimes \mathrm{id}\right) D_{0}+\frac{q^{2 n-1}}{\operatorname{dim}_{q} V} D_{0}^{\prime},
\end{aligned}
$$

where $\operatorname{dim}_{q} V=[n]_{q}\left(q^{n-1}+q^{1-n}\right)$ is the quantum dimension of $V$. Then

$$
F-q B=-q^{n} \frac{q^{n}-q^{-n}}{\operatorname{dim}_{q} V} D_{0}^{\prime}: V^{\otimes 3} \longrightarrow V^{\otimes 3} .
$$

Note that

$$
\begin{array}{rlrl}
\tau^{\otimes 2} \circ \iota_{(2,1)} \circ F\left(v_{a}\right) & =X_{1 a} \Psi^{(1,2)}, & \tau^{\otimes 2} \circ \iota_{(2,1)} \circ B\left(v_{a}\right)=\Psi^{(1,2)} X_{1 a}, \\
\text { and } \tau^{\otimes 2} \circ \iota_{(2,1)} \circ D_{0}^{\prime}\left(v_{a}\right) & =\Psi^{(1,1)} X_{2 a} .
\end{array}
$$

Thus for $i=1$ and $j=2$, we have

$$
q \Psi^{(i, j)} X_{i a}-X_{i a} \Psi^{(i, j)}=q^{n} \frac{q^{n}-q^{-n}}{\operatorname{dim}_{q} V} \Psi^{(i, i)} X_{j a} .
$$

This leads to the desired result taking into account that

$$
\Psi^{(i, i)}=\frac{q^{1-n} \operatorname{dim}_{q} V}{[n]_{q}} \bar{\psi}_{n}^{(i, i)},
$$

which is implied by (3.11). This completes the proof of the Lemma.

The following relations are easy consequences of part (2) of Lemma 3.7 . 


\section{Lemma 3.8.}

$$
\begin{aligned}
& \Psi^{(i, i)} \Psi^{(j, k)}-\Psi^{(j, k)} \Psi^{(i, i)}=0, \quad \text { for all } i, j, k, \\
& \Psi^{(i, k)} \Psi^{(i, j)}-q^{-1} \Psi^{(i, j)} \Psi^{(i, k)}=\left(q-q^{-1}\right) \bar{\psi}_{n}^{(i, i)} \Psi^{(j, k)}, \quad k \neq i, j ; i<j, \\
& \Psi^{(j, k)} \Psi^{(i, j)}-q^{-1} \Psi^{(i, j)} \Psi^{(j, k)}=\left(q-q^{-1}\right) \bar{\psi}_{n}^{(j, j)} \Psi^{(i, k)}, \quad k \neq i, j ; i<j, \\
& \Psi^{(i, j)} \Psi^{(k, l)}-\Psi^{(k, l)} \Psi^{(i, j)}=0, \quad k<i<j<l, \\
& \Psi^{(i, j)} \Psi^{(k, l)}-\Psi^{(k, l)} \Psi^{(i, j)} \\
& =\left(q-q^{-1}\right)\left(\Psi^{(i, k)} \Psi^{(j, l)}-\Psi^{(i, l)} \Psi^{(k, j)}\right), \quad i<k<j<l .
\end{aligned}
$$

3.3.1. The algebra $\mathrm{U}_{q}\left(\mathfrak{o}_{N}\right)$. Following [18] we introduce extra generators $\sigma^{ \pm 1}$ to augment $\mathrm{U}_{q}\left(\mathfrak{s o}_{N}\right)$, obtaining a new algebra, which we denote by $\mathrm{U}_{q}\left(\mathfrak{o}_{N}\right)$. Here $\sigma^{ \pm 1}$ are mutual inverses, and have the following properties: If $N$ is odd, $\sigma$ commutes with all the generators of $\mathrm{U}_{q}\left(\mathfrak{s o}_{N}\right)$. When $N=2 n$, we label the last two simple roots of $\mathfrak{g}$ as $\alpha_{n-1}=\epsilon_{n-1}-\epsilon_{n}$ and $\alpha_{n}=\epsilon_{n-1}+\epsilon_{n}$. Then

$$
\begin{array}{lll}
\sigma e_{n-1} \sigma^{-1}=e_{n}, & \sigma e_{n} \sigma^{-1}=e_{n-1}, \\
\sigma f_{n-1} \sigma^{-1}=f_{n}, & \sigma f_{n} \sigma^{-1}=f_{n-1}, \\
\sigma k_{n-1} \sigma^{-1}=k_{n}, & \sigma k_{n} \sigma^{-1}=k_{n-1},
\end{array}
$$

while all the other generators commute with $\sigma$. Denote by $\mathrm{U}_{q}\left(\mathfrak{o}_{N}\right)$ the associative algebra generated by $\mathrm{U}_{q}\left(\mathfrak{s o}_{N}\right)$ and $\sigma^{ \pm 1}$. We extend the co-multiplication and antipode of $\mathrm{U}_{q}\left(\mathfrak{s o}_{N}\right)$ to $\mathrm{U}_{q}\left(\mathfrak{o}_{N}\right)$ by letting $\Delta(\sigma)=\sigma \otimes \sigma$, and $S(\sigma)=\sigma^{-1}$. Then $\mathrm{U}_{q}\left(\mathfrak{o}_{N}\right)$ acquires the structure of a Hopf algebra. Now $\sigma$ acts as an automorphism on $\mathrm{U}_{q}\left(\mathfrak{s \mathfrak { s } _ { N }}\right)$ by conjugation. The $R$-matrix of $\mathrm{U}_{q}\left(\mathfrak{s o}_{N}\right)$ is invariant under the automorphism, i.e., $\Delta(\sigma)$ commutes with the $R$-matrix.

We extend the natural $\mathrm{U}_{q}\left(\mathfrak{s o}_{N}\right)$-module $V$ to a $\mathrm{U}_{q}\left(\mathfrak{o}_{N}\right)$-module by stipulating that

(i) For odd $n, \sigma$ acts on the highest weight vector of $V$ by -1 ;

(ii) For even $n, \sigma$ acts on the highest weight vector of $V$ by 1 .

Then $\mathrm{U}_{q}\left(\mathfrak{o}_{N}\right)$ also acts on tensor powers of $V$ through the co-multiplication. We shall consider the $\mathrm{U}_{q}\left(\mathfrak{o}_{N}\right)$-modules which are direct sums of submodules of $V^{\otimes r}$, $r \geq 0$.

Define $\mathrm{U}_{q}$-endomorphisms $b_{i}=\mathrm{id}_{V}^{\otimes(i-1)} \otimes \check{R} \otimes \mathrm{id}_{V}^{\otimes(r-1)}(1 \leq i \leq r-1)$ of $V^{\otimes r}$. These define a representation of the Birman-Wenzl-Murakami (BMW) algebra, and we denote by $\mathcal{B}(r)$ the image of the BMW algebra in this representation. Then we have the following result (see, e.g., [18]).

Theorem 3.9. If $V$ is the natural module for the quantum orthogonal group $\mathrm{U}_{q}\left(\mathfrak{o}_{N}\right)$, for any integer $r \geq 2$,

$$
\operatorname{End}_{\mathrm{U}_{q}\left(\mathfrak{o}_{N}\right)}\left(V^{\otimes r}\right)=\mathcal{B}(r) .
$$

3.4. Noncommutative FFT of invariant theory. The following result is a quantum analogue of the third (coordinate ring) formulation of the first fundamental theorem for the invariant theory of $\mathfrak{o}_{2 n}$.

Theorem 3.10. The subalgebra $\mathcal{A}_{m}^{\mathrm{U}_{q}\left(\mathfrak{o}_{2 n}\right)}:=\left\{f \in \mathcal{A}_{m} \mid x(f)=\epsilon(x) f, \forall x \in \mathrm{U}_{q}\left(\mathfrak{o}_{2 n}\right)\right\}$ of $\mathrm{U}_{q}\left(\mathfrak{o}_{2 n}\right)$-invariants in $\mathcal{A}_{m}$ is generated by the elements $\Psi^{(i, j)}(i \leq j)$ and the identity. 
Proof. Let $A=\mathcal{A}_{m}^{\mathrm{U}_{q}\left(\mathfrak{o}_{2 n}\right)}$ and $B=\mathcal{T}_{m}^{\mathrm{U}_{q}\left(\mathfrak{o}_{2 n}\right)}$, where $\mathcal{T}_{m}^{\mathrm{U}_{q}\left(\mathfrak{o}_{2 n}\right)}$ is the subalgebra of $\mathrm{U}_{q}\left(\mathfrak{o}_{2 n}\right)$-invariants of $\mathcal{T}_{m}$. Since both $\mathcal{T}_{m}$ and $\mathcal{A}_{m}$ are semi-simple as $\mathrm{U}_{q}$-modules, the surjection $\tau^{\otimes m}$ descends to $B$, giving rise to a surjective algebra homomorphism $B \longrightarrow A$.

Let $\mathbf{d}=\left(d_{1}, d_{2}, \ldots, d_{m}\right)$, and set

$$
\left(\mathcal{T}_{m}\right)_{\mathbf{d}}=T(V)_{d_{1}} \otimes \cdots \otimes T(V)_{d_{m}}, \quad\left(\mathcal{A}_{m}\right)_{\mathbf{d}}=S_{q}(V)_{d_{1}} \otimes \cdots \otimes S_{q}(V)_{d_{m}} .
$$

Then the homogeneous component $A_{\mathbf{d}}$ of $A$ of multi-degree $\mathbf{d}$ is

$$
A_{\mathbf{d}}=\left(\left(\mathcal{A}_{m}\right)_{\mathbf{d}}\right)^{\mathrm{U}_{q}}=\tau^{\otimes m}\left(\left(\left(\mathcal{T}_{m}\right)_{\mathbf{d}}\right)^{\mathrm{U}_{q}}\right)=\tau^{\otimes m}\left(B_{\mathbf{d}}\right),
$$

where $B_{\mathbf{d}}$ is the homogeneous subspace of $B$ of multi-degree $\mathbf{d}$. By considering the weights of $\left(\mathcal{T}_{m}\right)_{\mathbf{d}}$, one sees that $B_{\mathbf{d}}=0$ if $|d|=\sum d_{i}$ is odd. For $|d|=2 k$, we consider the $(0,2 k)$-tangle diagrams. The image of each diagram under the ReshetikhinTuraev functor is an element of $\operatorname{Hom}_{\mathrm{U}_{q}\left(\mathfrak{o}_{2 n}\right)}\left(\mathbb{C}(q), V^{\otimes 2 k}\right)$. Since (cf. \$2.4)

$$
\operatorname{Hom}_{\mathrm{U}_{q}\left(\mathfrak{o}_{2 n}\right)}\left(\mathbb{C}(q), V^{\otimes 2 k}\right) \cong \operatorname{End}_{\mathrm{U}_{q}\left(\mathfrak{o}_{2 n}\right)}\left(V^{\otimes k}\right) \text { as vector space, }
$$

the images of the $(0,2 k)$-tangle diagrams under the Reshetikhin-Turaev functor span $\operatorname{Hom}_{\mathrm{U}_{q}\left(\mathfrak{o}_{2 n}\right)}\left(\mathbb{C}(q), V^{\otimes 2 k}\right)$ by Theorem 3.9.

Note that there is a canonical isomorphism $\operatorname{Hom}_{\mathrm{U}_{q}\left(\mathfrak{o}_{2 n}\right)}\left(\mathbb{C}(q), V^{\otimes 2 k}\right) \stackrel{\sim}{\longrightarrow} B_{\mathbf{d}}$, given by $\phi \mapsto \phi(1)$. Thus we shall regard elements of $B$ as linear combinations of $(0,2 r)$ tangle diagrams for $r \geq 0$. Two tangle diagrams in $B$ may be multiplied in a natural way with the multiplication being that inherited from $\mathcal{T}_{m}$. We shall use this algebraic structure of $B$ presently.

Remark 3.11. It is important to note that this multiplication is different from the standard BMW-type multiplication obtained by composing diagrams, which has also arisen in this work. It is the quantum analogue of the commutative (pointwise) multiplication of coordinate functions in the classical case (i.e. setting three of the introduction), and changes degree. In terms of diagrams, it corresponds to setting diagrams side by side, rather than concatenating them.

Because the $\check{R}$-matrix in the natural $\mathrm{U}_{q}$-representation satisfies Kaufman's skein relation, all $(0,2 k)$-tangle diagrams can be expressed as linear combinations of diagrams with the property that any two strings cross each other at most once with an over (positive) crossing. A loop in such a tangle diagram can be replaced by a scalar factor equal to the quantum dimension $\operatorname{dim}_{q} V$ of $V$. Also if a string in such a tangle diagram has self-crossings, then the tangle diagram is proportional to that obtained by replacing this string by one without any self-crossing.

Given a $(0,2 k)$-tangle diagram in $B_{\mathbf{d}}$, we shall divide the interval $[1,2 k]$ into bands of length $d_{1}, d_{2}, \ldots$, with end points 1 to $d_{1}$ belonging to the first band, end points $\left(d_{1}+1\right)$ to $\left(d_{1}+d_{2}\right)$ to the second band, etc. If two strings cross each other and each has at least one end point belonging to the same band, we may replace the crossing (over crossing) by $q||-q^{1-n} \frac{q^{n}-q^{-n}}{\operatorname{dim}_{q} V} \asymp$. Then under the map $\tau^{\otimes m}$, the resulting linear combination of tangle diagrams yields the same element of $A_{\mathbf{d}}$ as the original diagram. Therefore, for the purpose of studying $A$, we may confine attention to tangle diagrams in $B$ satisfying the further condition that strings with end points belonging to the same band do not cross one another. 
To summarise, $A_{\mathbf{d}}$ is spanned by the elements $\tau^{\otimes m}(\phi)$, where $\phi$ is a $(0,2 k)$-tangle diagram in $B_{\mathbf{d}}$ which satisfies the following conditions:

(a) there are no loops;

(b) two strings cross each other at most once with an over crossing;

(c) no string crosses itself;

(d) strings sharing a band of end points do not cross one another.

As indicated above, this statement is based on the canonical identification $B_{\mathbf{d}} \stackrel{\sim}{\longrightarrow}$ $\operatorname{Hom}_{\mathrm{U}_{q}\left(\mathfrak{o}_{2 n}\right)}\left(\mathbb{C}(q), V^{\otimes 2 k}\right)$.

If $\zeta$ is a tangle diagram satisfying the conditions (a) to (d), it is evident that there exist tangle diagrams $\zeta_{1}, \ldots, \zeta_{t}$ such that $\zeta=\zeta_{1} \cdots \zeta_{t}$, where $\zeta_{i}$ has neither crossings nor loops, e.g., is of the form $\mathbb{V} \mathbb{W}$. Consider, by way of example, a $(0,4)$ tangle diagram with two strings such that the end points of one string are in bands 2 and 4 , while the end points of the other are in bands 1 and 3 . In this case, the tangle diagram is obviously the product of two $(0,2)$-tangle diagrams.

Recall that $\Psi^{(i, j)}$ commutes with all $X_{k a}$ for $k<i<j$ or $i<j<k$, and all $\Psi^{(i, i)}$ are central in $\mathcal{A}_{m}$. Thus $\tau^{\otimes m}\left(\zeta_{i}\right)$ can be expressed as $\tau^{\otimes m}\left(\zeta_{i, 1}\right) \tau^{\otimes m}\left(\zeta_{i, 2}\right) \cdots \tau^{\otimes m}\left(\zeta_{i, s}\right)$, where the tangle diagram $\zeta_{i, j}$ has neither crossings nor loops, and satisfies the condition that there are two distinct bands such that every string has an end point in each band.

Consider such a $\zeta_{i, j}$, the end points of whose strings belong to, say, bands $k$ and $l$. If there are only two strings in the diagram, that is, the diagram is of the form $\mathbb{V}$, then $\tau^{\otimes m}\left(\zeta_{i, j}\right)=\sum_{a, a} C_{a b} X_{k a} \Psi^{(k, l)} X_{l b}$, where $\Psi^{(k, l)}=\sum_{c, d} C_{c d} X_{k c} X_{l d}$. By the fourth relation of (3.18),

$$
\tau^{\otimes m}\left(\zeta_{i, j}\right)=q\left(\Psi^{(k, l)}\right)^{2}-\frac{\left(q-q^{-1}\right) q^{n-1}}{q^{n-1}+q^{1-n}} \Psi^{(k, k)} \Psi^{(l, l)}
$$

By induction on the number of strings in $\zeta_{i, j}, \tau^{\otimes m}\left(\zeta_{i, j}\right)$ can be expressed as a linear combination of monomials in $\Psi^{(k, l)}, \Psi^{(k, k)}$ and $\Psi^{(l, l)}$. This completes the proof of the theorem.

\section{INVARIANT THEORY OF THE QUANTUM ODD ORTHOGONAL GROUPS $\mathrm{U}_{q}\left(\mathfrak{s o}_{2 n+1}\right)$}

In this section we shall consider the invariant theory of $\mathrm{U}_{q}\left(\mathfrak{s o}_{2 n+1}\right)$. This case may be treated in almost identical fashion to the even orthogonal case, and we shall therefore present only the main lines of the argument, referring for details to the last section.

It will be convenient to work with a slightly modified version of the quantum group. If $e_{i}^{\prime}, f_{i}^{\prime}, k_{i}^{\prime \pm 1}(1 \leq i \leq n)$ are the generators of $\mathrm{U}_{q}\left(\mathfrak{s o}_{2 n+1}\right)$ in the standard Drinfeld-Jimbo presentation, where $e_{n}^{\prime}$ and $f_{n}^{\prime}$ are associated with the short simple root, we write $v=q^{\frac{1}{2}}$ and let

$$
\begin{aligned}
& e_{n}=\frac{e_{n}^{\prime}}{v+v^{-1}}, \\
& e_{i}=e_{i}^{\prime}, \quad 1 \leq i \leq n-1 ; \\
& k_{j}=k_{j}^{\prime}, \quad f_{j}=f_{j}^{\prime}, \quad 1 \leq j \leq n .
\end{aligned}
$$


Then the relations among the generators remain as in the standard presentation, except that now

$$
e_{i} f_{j}-f_{j} e_{i}=\delta_{i j} \frac{k_{i}-k_{i}^{-1}}{q-q^{-1}}, \quad \forall i
$$

This modified quantum group is defined over $\mathbb{C}(q)$, and many explicit formulae arising in its representation theory take a correspondingly simpler form.

In this section, $\mathrm{U}_{q}$ will denote this modified algebra.

4.1. Braided symmetric algebra of the natural $\mathrm{U}_{q}\left(\mathfrak{s o}_{2 n+1}\right)$-module. Denote by $V$ the natural module for $\mathrm{U}_{q}$. As above, denote by $\left\{v_{a} \mid a \in[1, n] \cup\{0\} \cup[-n,-1]\right\}$ a basis of weight vectors, where wt $v_{a}=\epsilon_{a}$. Let $E_{a b}$ be the matrix units in $\operatorname{End}(V)$ relative to this basis, and denote by $\pi: \mathrm{U}_{q} \longrightarrow \operatorname{End}(V)$ the irreducible representation of $\mathrm{U}_{q}$. Then relative to the above basis of $V, \pi$ may be realised as follows:

$$
\begin{aligned}
& \pi\left(e_{i}\right)=E_{i, i+1}-E_{-(i+1),-i}, \quad \pi\left(f_{i}\right)=E_{i+1, i}-E_{-i,-(i+1)}, \\
& \pi\left(e_{n}\right)=E_{n 0}-E_{0,-n}, \quad \pi\left(f_{n}\right)=E_{0 n}-E_{-n, 0}, \\
& \pi\left(k_{i}\right)=1+(q-1)\left(E_{i i}+E_{-(i+1),-(i+1)}\right)+\left(q^{-1}-1\right)\left(E_{i+1, i+1}+E_{-i,-i}\right), \\
& \pi\left(k_{n}\right)=1+(q-1) E_{n n}+\left(q^{-1}-1\right) E_{-n,-n} .
\end{aligned}
$$

The tensor product $V \otimes V$ decomposes as $L_{2 \epsilon_{1}} \oplus L_{\epsilon_{1}+\epsilon_{2}} \oplus L_{0}$; we have the following bases for the irreducible submodules.

(1) Basis for $L_{0}$ (cf. (2.14) ):

$$
\sum_{i=1}^{n}\left(q^{n-i} v_{i} \otimes v_{-i}+q^{i-n-1} v_{-i} \otimes v_{i}\right)+v_{0} \otimes v_{0} .
$$

(2) Basis for $L_{2 \epsilon_{1}}$ :

$$
\begin{aligned}
& v_{i} \otimes v_{i}, \quad v_{-i} \otimes v_{-i}, \quad 1 \leq i \leq n+1 \\
& v_{i} \otimes v_{j}+q v_{j} \otimes v_{i}, \quad v_{-j} \otimes v_{-i}+q v_{-i} \otimes v_{-j}, \quad i<j \leq n+1, \\
& v_{i} \otimes v_{-j}+q v_{-j} \otimes v_{i}, \quad i \neq j, \quad i, j \neq 0 \\
& (q+1) v_{0} \otimes v_{0}-q^{-1} v_{n} \otimes v_{-n}-q v_{-n} \otimes v_{n} \\
& q^{-1} v_{i} \otimes v_{-i}+q v_{-i} \otimes v_{i}-\left(v_{i+1} \otimes v_{-(i+1)}+v_{-(i+1)} \otimes v_{i+1}\right), \quad i \leq n-1,
\end{aligned}
$$

where $v_{n+1}$ is to be interpreted as $v_{0}$.

(3) Basis for $L_{\epsilon_{1}+\epsilon_{2}}$ :

$$
\begin{aligned}
& v_{i} \otimes v_{j}-q^{-1} v_{j} \otimes v_{i}, \quad v_{-j} \otimes v_{-i}-q^{-1} v_{-i} \otimes v_{-j}, \quad i<j \leq n+1, \\
& v_{i} \otimes v_{-j}-q^{-1} v_{-j} \otimes v_{i}, \quad i \neq j, i, j \neq 0, \\
& (q-11) v_{0} \otimes v_{0}-v_{n} \otimes v_{-n}+v_{-n} \otimes v_{n}, \\
& v_{i} \otimes v_{-i}-v_{-i} \otimes v_{i}-\left(q v_{i+1} \otimes v_{-i-1}-q^{-1} v_{-i-1} \otimes v_{i+1}\right), \quad i \leq n-1 .
\end{aligned}
$$

Let $P_{s}, P_{a}$ and $P_{0}$ respectively be the idempotent projection of $V \otimes V$ onto the irreducible submodule with highest weight $2 \epsilon_{1}, \epsilon_{1}+\epsilon_{2}$ and 0 . Then the $R$-matrix of $\mathrm{U}_{q}$ acting on $V \otimes V$ is given by

$$
\check{R}=q P_{s}-q^{-1} P_{a}+q^{-2 n} P_{0} .
$$


We also note that the quantum dimension of $V$ is given by

$$
\operatorname{dim}_{q} V=\left(q^{1-2 n}+1\right) \frac{q^{2 n}-q^{-1}}{q-q^{-1}} .
$$

The 'alternating' subspace' of the degree 2 homogeneous subspace $T(V)_{2}$ of the tensor algebra $T(V)$ of $V$ is in this case the submodule $L_{\epsilon_{1}+\epsilon_{2}}=P_{a}\left(T(V)_{2}\right)$. Let $\mathcal{I}_{q}$ be the two-sided ideal of $T(V)$ generated by $L_{\epsilon_{1}+\epsilon_{2}}$. Then the braided symmetric algebra of $V$ is defined by $S_{q}(V)=T(V) / \mathcal{I}_{q}$ (cf. \$2.2).

Lemma 4.1. The braided symmetric algebra $S_{q}(V)$ is generated by $v_{i}, v_{-i}(i \in[1, n])$ and $v_{n+1}:=v_{0}$ subject to the following relations

$$
\begin{aligned}
& v_{i} v_{j}-q^{-1} v_{j} v_{i}=0, \quad i<j \leq n+1, \\
& v_{-j} v_{-i}-q^{-1} v_{-i} v_{-j}=0, \quad i<j \leq n+1, \\
& v_{i} v_{-j}-q^{-1} v_{-j} v_{i}=0, \quad i \neq j, i, j \neq 0 \\
& (q-1) v_{0} v_{0}-v_{n} v_{-n}+v_{-n} v_{n}=0, \\
& v_{i} v_{-i}-v_{-i} v_{i}-\left(q v_{i+1} v_{-i-1}-q^{-1} v_{-i-1} v_{i+1}\right)=0, \quad i \leq n-1 .
\end{aligned}
$$

The defining relations are obtained using the basis of $L_{\epsilon_{1}+\epsilon_{2}}$ given above. From the last two relations we obtain

$$
v_{-i} v_{i}=v_{i} v_{-i}-\left(q-q^{-1}\right) q^{i+1-n} \phi_{i+1}^{(+)}-(q-1) q^{i-n}\left(v_{0}\right)^{2}, \quad \forall i \leq n,
$$

where $\phi_{i}^{(+)}=\sum_{k=i}^{n} q^{n-k} v_{k} v_{-k}$. From this one deduces that

$$
\sum_{i=1}^{n} q^{i-n-1} v_{-i} v_{i}=q^{1-2 n} \sum_{i=1}^{n} q^{n-i} v_{i} v_{-i}+\frac{(q-1)\left(q^{-2 n}-1\right)}{q-q^{-1}}\left(v_{0}\right)^{2} .
$$

Let $\Psi=: \sum_{i=1}^{n}\left(q^{n-i} v_{i} v_{-i}+q^{i-n-1} v_{-i} v_{i}\right)+\left(v_{0}\right)^{2}$, we have

$$
\Psi=\left(1+q^{1-2 n}\right)\left(\sum_{i=1}^{n} q^{n-j} v_{i} v_{-i}+\frac{1-q^{-1}}{q-q^{-1}}\left(v_{0}\right)^{2}\right) .
$$

In analogy with the case of the even dimensional quantum orthogonal group, we have the following result.

Theorem 4.2. (1) The ordered monomials in $v_{i}, v_{-i}(i \in[1, n])$ and $v_{0}$ form a basis of $S_{q}(V)$.

(2) The element $\Psi$ is invariant under $\mathrm{U}_{q}\left(\mathfrak{s o}_{2 n+1}\right)$ and belongs to the centre of $S_{q}(V)$.

(3) The subalgebra $S_{q}(V)^{\mathrm{U}_{q}\left(\mathfrak{s o}_{2 n+1}\right)}$ of invariants is generated by $\Psi$, and thus is isomorphic to a polynomial algebra in one variable.

Proof. The proof is essentially the same as that in the case of the even dimensional quantum orthogonal group. Note that part (1) is known from [3].

This permits the definition of the even dimensional quantum sphere, in analogy with Lemma 3.5 .

Corollary 4.3. Let $\langle\Psi-1\rangle$ be the two-sided ideal of $S_{q}(V)$ generated by $\Psi-1$, and define the quantum sphere $\mathbb{S}_{q}^{2 n}=S_{q}(V) /\langle\Psi-1\rangle$. Then $\mathbb{S}_{q}^{2 n}$ is a module algebra for $\mathrm{U}_{q}\left(\mathfrak{s o}_{2 n+1}\right)$ and $\mathbb{S}_{q}^{2 n}=\bigoplus_{k=0}^{\infty} L_{k \epsilon_{1}}$. 
4.2. A $\mathrm{U}_{q}\left(\mathfrak{s o}_{2 n+1}\right)$-module algebra and the FFT of invariant theory. The quantum analogue of the coordinate ring of $\oplus^{m} V$ is the associative algebra $\mathcal{A}_{m}=$ $\left(S_{q}(V)^{\otimes m}, \mu_{S}\right)$ (see (2.6) ), constructed by iterating the procedure of Theorem 2.3 . It will be convenient to relabel the basis elements of $V$ as follows. Write $v_{n+1}$ for $v_{0}$, and $v_{2 n+2-t}$ for $v_{-t}$ for all $t \in[1, n]$. As in $\S 3.3$, define elements $X_{i a} \in \mathcal{A}_{m}$ for $i \in[1, m]$ and $a \in[1,2 n+1]$. A presentation for $\mathcal{A}_{m}$ may be obtained as in Lemma 3.6.

Lemma 4.4. The $\mathrm{U}_{q}\left(\mathfrak{s o}_{2 n+1}\right)$-module algebra $\mathcal{A}_{m}$ is generated by $X_{\text {ia }}$ with $i \in[1, m]$ and $a \in[1,2 n+1]$, subject to the following relations:

(1) for each $i$, the elements $X_{i a}$ obey the relations (4.2) with $v_{a}$ repalced by $X_{i a}$;

(2) for $i<j$ and $a, b \in[1,2 n+1]$,

$$
\begin{aligned}
X_{j a} X_{i a} & =q X_{i a} X_{j a}, \quad a \in[1,2 n+1] \\
X_{j b} X_{i a} & =X_{i a} X_{j b}+\left(q-q^{-1}\right) X_{i b} X_{j a}, \\
X_{j a} X_{i b} & =X_{i b} X_{j a}, \quad a<b, a+b \neq 2 n+2, \\
X_{j, n+1} X_{i, n+1} & =X_{i, n+1} X_{j, n+1}-\left(q-q^{-1}\right) \psi_{n}^{(i, j)}, \\
X_{j t} X_{i, 2 n+2-t} & =q X_{i, 2 n+2-t} X_{j t}-\left(q-q^{-1}\right) q^{n-t+1} \psi_{t}^{(i, j)}, \\
X_{j, 2 n+2-t} X_{i t} & =q^{-1} X_{i t} X_{j, 2 n+2-t}-\left(q-q^{-1}\right) X_{i, 2 n+2-t} X_{j t} \\
& +\left(q-q^{-1}\right) q^{t-n}\left(\bar{\psi}_{t}^{(i, j)}-\Psi^{(i, j)}\right), \quad t \in[1, n],
\end{aligned}
$$

where (for all $i, j \in[1, m]$ )

$$
\begin{aligned}
\psi_{t}^{(i, j)} & =\sum_{s=1}^{t} q^{s-n-1} X_{i, 2 n+2-s} X_{j s}, \\
\bar{\psi}_{t}^{(i, j)} & =\sum_{s=1}^{t} q^{n-s} X_{i s} X_{j, 2 n+2-s}, \\
\Psi^{(i, j)} & =\psi_{n}^{(i, j)}+X_{i, n+1} X_{j, n+1}+\bar{\psi}_{n}^{(i, j)} .
\end{aligned}
$$

These relations are obtained in the same way as in the case of $\mathrm{U}_{q}\left(\mathfrak{s o}_{2 n}\right)$. The elements $\Psi^{(i, j)}(i \leq j)$ will play a special role in the study of invariants below; we therefore record the following relations.

Lemma 4.5. (1) For all $i, j \in[1, m], \Psi^{(i, j)}$ are $\mathrm{U}_{q}$-invariant and satisfy

$$
\Psi^{(j, i)}=q^{-2 n} \Psi^{(i, j)} \quad \text { if } \quad i<j .
$$

(2) The elements $\Psi^{(i, j)}$ satisfy the following relations:

$$
\begin{aligned}
& X_{k a} \Psi^{(i, i)}-\Psi^{(i, i)} X_{k a}=0, \quad \text { for all } i, k, \\
& X_{k a} \Psi^{(i, j)}-\Psi^{(i, j)} X_{k a}=0, \quad k>i, j \quad \text { or } k<i, j, \\
& X_{k a} \Psi^{(i, j)}-\Psi^{(i, j)} X_{k a} \\
& =\left(q-q^{-1}\right)\left(X_{i a} \Psi^{(k, j)}-\Psi^{(i, k)} X_{j a}\right), \quad i<k<j, \\
& \Psi^{(i, j)} X_{i a}-q^{-1} \Psi^{(i, j)} X_{i a}=\left(q-q^{-1}\right) \varphi^{(i, i)} X_{j a}, \quad i<j, \\
& X_{j a} \Psi^{(i, j)}-q^{-1} \Psi^{(i, j)} X_{j a}=\left(q-q^{-1}\right) \varphi^{(j, j)} X_{i a}, \quad i<j .
\end{aligned}
$$


where

$$
\varphi^{(i, i)}:=\frac{q^{2 n}-q^{-1}}{q-q^{-1}} \Psi^{(i, i)}=\bar{\psi}_{n}^{(i, i)}+\frac{1-q^{-1}}{q-q^{-1}}\left(X_{i, n+1}\right)^{2}
$$

It follows from part (2) of Lemma 4.5 that the elements $\Psi^{(i, j)}$ also satisfy the following relations:

\section{Lemma 4.6.}

$$
\begin{aligned}
& \Psi^{(i, i)} \Psi^{(j, k)}-\Psi^{(j, k)} \Psi^{(i, i)}=0, \quad \text { for all } i, j, k, \\
& \Psi^{(i, k)} \Psi^{(i, j)}-q^{-1} \Psi^{(i, j)} \Psi^{(i, k)}=\left(q-q^{-1}\right) \varphi^{(i, i)} \Psi^{(j, k)}, \quad k \neq i, j ; i<j, \\
& \Psi^{(j, k)} \Psi^{(i, j)}-q^{-1} \Psi^{(i, j)} \Psi^{(j, k)}=\left(q-q^{-1}\right) \varphi^{(i, i)} \Psi^{(i, k)}, \quad k \neq i, j ; i<j, \\
& \Psi^{(i, j)} \Psi^{(k, l)}-\Psi^{(k, l)} \Psi^{(i, j)}=0, \quad k<i<j<l, \\
& \Psi^{(i, j)} \Psi^{(k, l)}-\Psi^{(k, l)} \Psi^{(i, j)} \\
& =\left(q-q^{-1}\right)\left(\Psi^{(i, k)} \Psi^{(j, l)}-\Psi^{(i, l)} \Psi^{(k, j)}\right), \quad i<k<j<l .
\end{aligned}
$$

We now express our results as a quantum analogue of the classical case. Consider the quantum orthogonal algebra $\mathrm{U}_{q}\left(\mathfrak{o}_{2 n+1}\right)$ as defined in [18] and discussed in $\$ 3.3 .1$. Extend the natural $\mathrm{U}_{q}\left(\mathfrak{s o}_{2 n+1}\right)$-module $V$ to a $\mathrm{U}_{q}\left(\mathfrak{o}_{2 n+1}\right)$-module as indicated there. The following result is our quantum analogue of the polynomial form of the first fundamental theorem for the invariant theory of $\mathfrak{o}_{2 n+1}$. Its proof is the same as that of Theorem 3.10 .

Theorem 4.7. The subalgebra $\mathcal{A}_{m}^{\mathrm{U}_{q}\left(\mathfrak{o}_{2 n+1}\right)}:=\left\{f \in \mathcal{A}_{m} \mid x(f)=\epsilon(x) f, \forall x \in\right.$ $\left.\mathrm{U}_{q}\left(\mathfrak{o}_{2 n+1}\right)\right\}$ of $\mathrm{U}_{q}\left(\mathfrak{o}_{2 n+1}\right)$-invariants in $\mathcal{A}_{m}$ is generated by the elements $\Psi^{(i, j)}(i \leq j)$ and the identity. These generators satisfy the commutation relations (4.6).

\section{INVARIANT THEORY FOR THE QUANTUM SYMPLECTIC GROUPS $\mathrm{U}_{q}\left(\mathfrak{s p}_{2 n}\right)$}

We now turn to the invariant theory of the quantum symplectic group. In this section $\mathrm{U}_{q}$ will denote $\mathrm{U}_{q}\left(\mathfrak{s p}_{2 n}\right)$.

5.1. Braided symmetric algebra of the natural $\mathrm{U}_{q}\left(\mathfrak{s p}_{2 n}\right)$-module. Let $V$ be the natural module for $\mathrm{U}_{q}$. As in the orthogonal cases, $\left\{v_{a} \mid a \in[1, n] \cup[-n,-1]\right\}$ denotes a basis of weight vectors, with the usual notation for weights, and $E_{a b}$ are the matrix units in $\operatorname{End}(V)$ with respect to this basis. Denote by $\pi: \mathrm{U}_{q}\left(\mathfrak{s p}_{2 n}\right) \longrightarrow$ $\operatorname{End}(V)$ the irreducible representation of $\mathrm{U}_{q}\left(\mathfrak{s p}_{2 n}\right)$ in $V$. Then relative to the above basis, we may realise $\pi$ as follows. In the formulae below, $i<n$.

$$
\begin{aligned}
& \pi\left(e_{i}\right)=E_{i, i+1}-E_{-i-1,-i}, \quad \pi\left(f_{i}\right)=E_{i, i+1}-E_{-i,-i-1}, \\
& \pi\left(e_{n}\right)=E_{n,-n}, \quad \pi\left(f_{n}\right)=E_{-n, n}, \\
& \pi\left(k_{i}\right)=1+(q-1)\left(E_{i i}+E_{-i-1,-i-1}\right)+\left(q^{-1}-1\right)\left(E_{i+1, i+1}+E_{-i,-i}\right), \\
& \pi\left(k_{n}\right)=1+\left(q^{2}-1\right) E_{n n}+\left(q^{-2}-1\right) E_{-n,-n} .
\end{aligned}
$$

For later use, we again provide explicit bases for the irreducible submodules of $V \otimes V \simeq L_{0} \oplus L_{2 \epsilon_{1}} \oplus L_{\epsilon_{1}+\epsilon_{2}}$. 
(1) Basis of $L_{0}$ :

$$
\sum_{i=1}^{n}\left(q^{n-i+1} v_{i} \otimes v_{-i}-q^{i-n-1} v_{-i} \otimes v_{i}\right)
$$

(2) Basis of $L_{2 \epsilon_{1}}(i, j \in[1, n]$ below):

$$
\begin{aligned}
& v_{a} \otimes v_{a}, \quad a \in[1, n] \cup[-n,-1], \\
& v_{i} \otimes v_{j}+q v_{j} \otimes v_{i}, \quad v_{-j} \otimes v_{-i}+q v_{-i} \otimes v_{-j}, \quad i<j, \\
& v_{i} \otimes v_{-j}+q v_{-j} \otimes v_{i}, \quad i \neq j, \\
& v_{i+1} \otimes v_{-i-1}+v_{-i-1} \otimes v_{i+1}-\left(q^{-1} v_{i} \otimes v_{-i}+q v_{-i} \otimes v_{i}\right), \quad i<n ; \\
& q^{-1} v_{n} \otimes v_{-n}+q v_{-n} \otimes v_{n} .
\end{aligned}
$$

(3) Basis of $L_{\epsilon_{1}+\epsilon_{2}}(i, j \in[1, n]$ below):

$$
\begin{aligned}
& v_{i} \otimes v_{j}-q^{-1} v_{j} \otimes v_{i}, \quad v_{-j} \otimes v_{-i}-q^{-1} v_{-i} \otimes v_{-j}, \quad i<j, \\
& v_{i} \otimes v_{-j}-q^{-1} v_{-j} \otimes v_{i}, \quad i \neq j, \\
& v_{i} \otimes v_{-i}-v_{-i} \otimes v_{i}-\left(q v_{i+1} \otimes v_{-i-1}-q^{-1} v_{-i-1} \otimes v_{i+1}\right), \quad i<n .
\end{aligned}
$$

Denote by $P_{s}, P_{a}$ and $P_{0}$ the idempotent projections from $V \otimes V$ onto the irreducible submodule with highest weight $2 \epsilon_{1}, \epsilon_{1}+\epsilon_{2}$ and 0 respectively. Then one checks easily (cf. [18, (6.10)]) that the $R$-matrix of $\mathrm{U}_{q}$ acting on $V \otimes V$ is given by

$$
\check{R}=q P_{s}-q^{-1} P_{a}-q^{-2 n-1} P_{0} .
$$

The 'alternating subspace' of the tensor square $T_{2}$ of $V$ is this case therefore equal to $P_{a}\left(T_{2}\right) \oplus P_{0}\left(T_{2}\right)$. This subspace generates a graded two-sided ideal $\mathcal{I}_{q}$ of $T(V)$, and as in $\$ 2.2$ the braided symmetric algebra of $V$ is $S_{q}(V)=T(V) / \mathcal{I}_{q}$; the map $\tau: T(V) \longrightarrow S_{q}(V)$ is the natural surjection. As we have seen, $S_{q}(V)$ has the structure of a $\mathrm{U}_{q}$-module algebra.

The following result is easily proved using the above explicit bases.

Lemma 5.1. The braided symmetric algebra $S_{q}(V)$ is generated by $\left\{v_{i}, v_{-i} \mid i \in\right.$ $[1, n]\}$ subject to the following relations:

$$
\begin{aligned}
& v_{i} v_{j}-q^{-1} v_{j} v_{i}=0, \quad v_{-j} v_{-i}-q^{-1} v_{-i} v_{-j}=0, \quad i<j, \\
& v_{i} v_{-j}-q^{-1} v_{-j} v_{i}=0, \quad i \neq j, \\
& v_{i} v_{-i}-v_{-i} v_{i}-\left(q v_{i+1} v_{-i-1}-q^{-1} v_{-i-1} v_{i+1}\right)=0, \quad i<n, \\
& \sum_{i=1}^{n}\left(q^{n-i+1} v_{i} v_{-i}-q^{i-n-1} v_{-i} v_{i}\right)=0 .
\end{aligned}
$$

Write $\psi_{i}=\sum_{j=1}^{i} q^{n+1-j} v_{-i} v_{i}$. The last two relations are equivalent to

$$
\begin{aligned}
& v_{-i} v_{i}=q^{2} v_{i} v_{-i}+\left(q-q^{-1}\right) q^{i-n} \psi_{i-1}, \quad i<n, \\
& v_{-n} v_{n}=v_{n} v_{-n}+\left(q-q^{-1}\right) \psi_{n} .
\end{aligned}
$$

It is known [3, 34] that $S_{q}(V)$ is flat, so that the degree $k$ homogeneous subspace $S_{q}(V)_{k}$ has the same dimension as that of $S(V)_{k}$, which is equal to $\left[\begin{array}{c}2 n+k-1 \\ k\end{array}\right]$. Since $\left(v_{1}\right)^{k}$ evidently generates an irreducible $\mathrm{U}_{q}$-submodule with highest weight 
$k \epsilon_{1}$, and this submodule has the same dimension, it follows that $S_{q}(V)_{k} \simeq L_{k \epsilon_{1}}$, and hence that $S_{q}(V)=\oplus_{k=0}^{\infty} L_{k \epsilon_{1}}$ as a $\mathrm{U}_{q}$-module. The next statement is now clear.

Lemma 5.2. The subalgebra $S_{q}(V)^{\mathrm{U}_{q}\left(\mathfrak{s p}_{2 n}\right)}$ of invariants is equal to $\mathcal{K}$.

5.2. A $\mathrm{U}_{q}\left(\mathfrak{s p}_{2 n}\right)$-module algebra and the FFT of invariant theory. As in the case of the orthogonal groups, we take as the non-commutative analogue of the coordinate ring of $\oplus^{m} V$ the $\mathrm{U}_{q}$-module algebra $\mathcal{A}_{m}=S_{q}(V)^{\otimes m}$ with multiplication obtained by iterating (2.4). As in Lemma 2.9, $\tau^{\otimes m}: \mathcal{T}_{m} \longrightarrow \mathcal{A}_{m}$ is a surjective homomorphism of $\mathrm{U}_{q}$-algebras.

A presentation for $\mathcal{A}_{m}$ may be obtained by calculations analogous to those in Lemma 3.6. Define $X_{i a}$ with $1 \leq i \leq m$ and $a \in[-n,-1] \cup[1, n]$ in analogous fashion to (2.7). For all $r, s \in[1, m]$, let

$$
\begin{aligned}
\bar{\psi}_{i}^{(r, s)} & =\sum_{i=1}^{n} q^{n+1-i} X_{r i} X_{s,-i} \\
\psi_{i}^{(r, s)} & =\sum_{i=1}^{n} q^{i-n-1} X_{r,-i} X_{s i} \\
\Psi^{(s, t)} & =\sum_{i=1}^{n}\left(q^{n+1-i} X_{s i} X_{t,-i}-q^{i-n-1} X_{s,-i} X_{t i}\right) .
\end{aligned}
$$

Lemma 5.3. The algebra $\mathcal{A}_{m}$ is generated by the elements $X_{i a}(1 \leq i \leq m, 1 \leq$ $a \leq 2 n)$ subject to the following relations:

(1) for each $i$, the elements $X_{i a}$ obey the relations (5.1) with $v_{a}$ replaced by $X_{i a}$;

(2) for $s<t \in[1, m], a, b \in[1,2 n]$ and $i \in[1, n]$,

$$
\begin{aligned}
& X_{t a} X_{s a}=q X_{s a} X_{t a}, \\
& X_{t a} X_{s b}=X_{s b} X_{t a}, \quad a<b \neq 2 n+1-a, \\
& X_{t b} X_{s a}=X_{s a} X_{t b}+\left(q-q^{-1}\right) X_{s b} X_{t a}, \quad a<b \neq 2 n+1-a ; \\
& \Psi^{(t, s)}=-q^{-1-2 n} \Psi^{(s, t)} ; \\
& X_{t i} X_{s, 2 n+1-i}=q^{-1} X_{s, 2 n+1-i} X_{t i}, \\
& X_{t, 2 n+1-i} X_{s i}= \\
& q X_{s i} X_{t, 2 n+1-i}+\left(q-q^{-1}\right) X_{s, 2 n+1-i} X_{t i} \\
& +\left(q-q^{-1}\right) q^{i-n-1}\left(\bar{\psi}_{i+1}^{(s, t)}-\Psi^{(s, t)}\right),
\end{aligned}
$$

where $\psi_{n+1}^{(s, t)}=0$ by convention.

We now turn to the study of the subalgebra

$$
\mathcal{A}_{m}^{\mathrm{U}_{q}\left(\mathfrak{s p}_{2 n}\right)}:=\left\{f \in \mathcal{A}_{m} \mid x(f)=\epsilon(x) f, \forall x \in \mathrm{U}_{q}\left(\mathfrak{s p}_{2 n}\right)\right\}
$$

of $\mathrm{U}_{q}\left(\mathfrak{s p}_{2 n}\right)$ invariants in $\mathcal{A}_{m}$. We shall need the following result. 
Lemma 5.4. The elements $\Psi^{(i, j)}$ are $\mathrm{U}_{q}\left(\mathfrak{s p}_{2 n}\right)$-invariant, and the following relations hold in $\mathcal{A}_{m}$.

$$
\begin{aligned}
& X_{k a} \Psi^{(i, j)}-\Psi^{(i, j)} X_{k a}=0, \quad k<i<j \text { or } i<j<k \\
& X_{k a} \Psi^{(i, j)}-\Psi^{(i, j)} X_{k a}=\left(q-q^{-1}\right)\left(X_{i a} \Psi^{(k, j)}+\Psi^{(i, k)} X_{j a}\right), \quad i<k<j, \\
& X_{i a} \Psi^{(i, j)}-q \Psi^{(i, j)} X_{i a}=0, \quad i<j, \\
& \Psi^{(i, j)} X_{j a}-q X_{j a} \Psi^{(i, j)}=0, \quad i<j .
\end{aligned}
$$

Proof. The invariance of the elements $\Psi^{(i, j)}$ is clear. Moreover the first and second relations are proved in exactly the same way as the second and third relations in (3.18), using the skein relations satisfied by the $R$-matrices.

To prove the other two relations, we need only consider the case $i=1$ and $j=2$. The restrictions of $\tau^{\otimes 2}$ to the subspaces $1 \otimes T(V)_{1} \oplus T(V)_{1} \otimes 1$ and $T(V)_{1} \otimes T(V)_{1}$ are all injective. Hence we have unique preimages $\hat{X}_{i a} \in 1 \otimes T(V)_{1} \oplus T(V)_{1} \otimes 1$ $(i=1,2)$ and $\hat{\Psi}^{(1,2)} \in T(V)_{1} \otimes T(V)_{1}$ of $X_{i a}$ and $\Psi^{(1,2)}$ respectively. Write $\hat{\Psi}^{(1,2)}=$ $\sum_{a, b} C_{a b} \hat{X}_{1 a} \hat{X}_{2 b}$, where $C_{a b} \in \mathcal{K}$, and let $\alpha_{t}, \beta_{t} \in \mathrm{U}_{q}$ be such that the universal $R$-matrix $R=\sum_{t} \alpha_{t} \otimes \beta_{t}$. Then

$$
\hat{\Psi}^{(1,2)} \hat{X}_{1 c}=\sum_{t} \sum_{a, b} C_{a b} \hat{X}_{1 a} \beta_{t}\left(\hat{X}_{1 c}\right) \alpha_{t}\left(\hat{X}_{2 b}\right),
$$

Write $R^{-1}=\sum_{t} \bar{\alpha}_{t} \otimes \bar{\beta}_{t}$. Then using the defining property $(\Delta \otimes \mathrm{id}) R=R_{13} R_{23}$ of $R$ and also the fact that $\hat{\Psi}^{(1,2)}$ is $\mathrm{U}_{q^{-}}$-invariant, one shows that

$$
\hat{\Psi}^{(1,2)} \hat{X}_{1 c}=\sum_{t} \sum_{a, b} C_{a b} \bar{\alpha}_{t}\left(\hat{X}_{1 a}\right) \bar{\beta}_{t}\left(\hat{X}_{1 c}\right) \hat{X}_{2 b}
$$

It follows from the definition of $S_{q}(V)$ that $\tau \check{R}\left(t_{2}\right)=q \tau\left(t_{2}\right)$ and $\tau \check{R}^{-1}\left(t_{2}\right)=q^{-1} \tau\left(t_{2}\right)$ for all $t_{2} \in T(V)_{2}$. Thus

$$
\tau^{\otimes 2}\left(\hat{\Psi}^{(1,2)} \hat{X}_{1 c}\right)=q^{-1} \tau^{\otimes 2}\left(\sum_{a, b} C_{a b} \hat{X}_{1 c} \hat{X}_{1 a} \hat{X}_{2 b}\right)=\tau^{\otimes 2}\left(\hat{X}_{1 c} \hat{\Psi}^{(1,2)}\right) .
$$

The third relation of (5.6) follows immediately, and the fourth relation can be proved similarly.

It is straightforward to derive the following commutation relations among the elements $\Psi^{(i, j)}$ from Lemma 5.4.

Proposition 5.5. Assume that $i<j$ and $k<l$.

(1) If $l \neq j$, then $\Psi^{(i, j)} \Psi^{(i, l)}=q^{-1} \Psi^{(i, l)} \Psi^{(i, j)}$.

(2) If $i<k$, then $\Psi^{(i, j)} \Psi^{(k, j)}=q^{-1} \Psi^{(k, j)} \Psi^{(i, j)}$.

(3) If $i<k$ and $j>l$, then $\Psi^{(i, j)} \Psi^{(k, l)}=\Psi^{(k, l)} \Psi^{(i, j)}$.

(4) If $i<k$ and $j<l$, then

$$
\Psi^{(k, l)} \Psi^{(i, j)}-\Psi^{(i, j)} \Psi^{(k, l)}=\left(q-q^{-1}\right)\left(\Psi^{(i, l)} \Psi^{(k, j)}+\Psi^{(i, k)} \Psi^{(j, l)}\right) .
$$

The following result is the quantum analogue of the first fundamental theorem of invariant theory for $\mathrm{U}_{q}\left(\mathfrak{s p}_{2 n}\right)$. Its proof is a straightforward adaptation of that of Theorem 3.10,

Theorem 5.6. The subalgebra $\mathcal{A}_{m}^{\mathrm{U}_{q}\left(\mathfrak{s p}_{2 n}\right)}$ of $\mathrm{U}_{q}\left(\mathfrak{s p}_{2 n}\right)$-invariants in $\mathcal{A}_{m}$ is generated by the elements $\Psi^{(i, j)}(i<j)$ and the identity. 


\section{INVARIANT THEORY FOR THE QUANTUM GENERAL LINEAR GROUP}

In this section, we study the invariant theory of the quantum general linear group. Our first requirement is a quantum analogue of $S\left(\oplus^{k} V \oplus^{l} V^{*}\right)$, where $V$ is the natural $\mathfrak{g l}_{n}$-module and $V^{*}$ is its dual. There are various ways this could be approached, all of which turn out to be equivalent.

One way is to follow the construction of Section 2 to first construct the braided symmetric algebras $S_{q}(V)$ and $S_{q}\left(V^{*}\right)$ over $\mathrm{U}_{q}\left(\mathfrak{g l}_{n}\right)$, and then to form the module algebras $S_{q}(V)^{\otimes k}$ and $S_{q}\left(V^{*}\right)^{\otimes l}$. From these, one constructs the module algebra $S_{q}(V)^{\otimes k} \otimes S_{q}\left(V^{*}\right)^{\otimes l}$ using Theorem 2.3, and this is a quantum analogue of the symmetric algebra over $S\left(\oplus^{k} V \oplus^{l} V^{*}\right)$.

Another possibility is to directly construct quantum analogues of the symmetric algebras of $\oplus^{k} V$ and of $\oplus^{l} V^{*}$. In this case, we need to consider the Lie algebras $\mathfrak{g l}_{n} \times \mathfrak{g l}_{k}$ and $\mathfrak{g l}_{n} \times \mathfrak{g l}_{l}$. Let $\mathfrak{g}=\mathfrak{g l}_{n} \times \mathfrak{g l}_{k}$, and let $W=V \otimes V^{(k)}$, where $V$ and $V^{(k)}$ are respectively the natural modules for $\mathrm{U}_{q}\left(\mathfrak{g l}_{n}\right)$ and $\mathrm{U}_{q}\left(\mathfrak{g l}_{k}\right)$. Then $W$ is an irreducible $\mathrm{U}_{q}(\mathfrak{g})$-module, and following $₫ 2$ we may form the braided symmetric algebra $S_{q}(W)$. It is known [34] that $W$ is one of the extremely rare modules such that $S_{q}(W)$ is flat. That is, $S_{q}(W)$ is (linearly) isomorphic to $S_{q}(V)^{\otimes k}$. We may similarly consider the irreducible $\mathrm{U}_{q}(\mathfrak{g})$-module $W^{\prime}=V^{*} \otimes V^{(l)}$, where now $\mathfrak{g}=\mathfrak{g l}_{n} \otimes \mathfrak{g l}_{l}$, and construct $S_{q}\left(W^{\prime}\right)$. Then using Theorem 2.3 we obtain the module algebra $S_{q}(W) \otimes S_{q}\left(W^{\prime}\right)$ over $\mathrm{U}_{q}\left(\mathfrak{g l}_{n}\right)$.

The above two constructions are essentially equivalent, and are equivalent to a third construction, which is conceptually simpler, and is the one we shall use in the present work. We now discuss this third construction.

6.1. Algebra of functions on the quantum general linear group. The quantum general linear group $\mathrm{U}_{q}\left(\mathfrak{g l}_{N}\right)$ is generated by $K_{b}^{ \pm 1}(1 \leq b \leq N)$, e $e_{a}$ and $f_{a}$ $(1 \leq a<N)$. The defining relations are essentially the same as those for $\mathrm{U}_{q}\left(\mathfrak{s l}_{N}\right)$ except for those involving the elements $K_{b}^{ \pm 1}$, which are given by

$$
\begin{aligned}
& K_{b} e_{a} K_{b}^{-1}=\left(1+(q-1) \delta_{b a}+\left(q^{-1}-1\right) \delta_{b, a+1}\right) e_{a}, \\
& K_{b} f_{a} K_{b}^{-1}=\left(1+(q-1) \delta_{b, a+1}+\left(q^{-1}-1\right) \delta_{b a}\right) f_{a}, \\
& e_{a} f_{b}-f_{b} e_{a}=\delta_{a b} \frac{K_{a} K_{a+1}^{-1}-K_{a+1} K_{a}^{-1}}{q-q^{-1}} .
\end{aligned}
$$

Let $\left\{v_{a} \mid 1 \leq a \leq N\right\}$ be the standard basis of weight vectors of the natural module $V^{(N)}$ for $\mathrm{U}_{q}\left(\mathfrak{g l}_{N}\right)$, with $\operatorname{wt}\left(v_{i}\right)=\epsilon_{i}$, and let $\pi: \mathrm{U}_{q}\left(\mathfrak{g l}_{N}\right) \longrightarrow \operatorname{End}\left(V^{(N)}\right)$ be the usual representation of $\mathrm{U}_{q}\left(\mathfrak{g l}_{N}\right)$ relative to this basis. Define elements $t_{a b}$ $(1 \leq a, b \leq N)$ of the dual vector space $\mathrm{U}_{q}\left(\mathfrak{g l}_{N}\right)^{*}$ of $\mathrm{U}_{q}\left(\mathfrak{g l}_{N}\right)$ by $\left\langle t_{a b}, x\right\rangle=\pi(x)_{a b}$ (the $(a, b)$ entry of the matrix $\pi(x)), \forall x \in \mathrm{U}_{q}\left(\mathfrak{g l}_{N}\right)$, and call these the cooordinate functions of the representation $\pi$.

Now the dual space of $\mathrm{U}_{q}\left(\mathfrak{g l}_{N}\right)$ has a natural algebraic structure with multiplication defined as follows. For any $t, t^{\prime} \in \mathrm{U}_{q}\left(\mathfrak{g l}_{N}\right)^{*},\left\langle t t^{\prime}, x\right\rangle=\sum_{(x)}\left\langle t \otimes t^{\prime}, x_{(1)} \otimes x_{(2)}\right\rangle$, for all $x \in \mathrm{U}_{q}\left(\mathfrak{g l}_{N}\right)$. Following an idea of [32], we consider the subalgebra $\mathcal{M}_{N}$ of $\mathrm{U}_{q}\left(\mathfrak{g l}_{N}\right)^{*}$ generated by the elements $t_{a b}(a, b \in[1, N])$. Then $\mathcal{M}_{N}$ has a presentation 
with generators $t_{a b}(a, b \in[1, N])$ and relations

$$
\sum_{a^{\prime}, b^{\prime}} R_{a a^{\prime}, b b^{\prime}} t_{a^{\prime} c} t_{b^{\prime} d}=\sum_{a^{\prime}, b^{\prime}} t_{b b^{\prime}} t_{a a^{\prime}} R_{a^{\prime} c, b^{\prime} d}
$$

where the $R_{a a^{\prime}, b b^{\prime}}$ are the entries of the $R$-matrix, which in our case has the form

$$
R=1 \otimes 1+(q-1) \sum_{a=1}^{N} E_{a a} \otimes E_{a a}+\left(q-q^{-1}\right) \sum_{a<b} E_{a b} \otimes E_{b a} .
$$

This algebra has been studied extensively in the literature (cf., e.g. [33, 4, 13]). We shall recall a few relevant facts. The algebra $\mathcal{M}_{N}$ is $\mathbb{Z}_{+}$-graded with $t_{a b}$ of degree 1. It has the structure of a bi-algebra with co-multiplication given by

$$
\Delta\left(t_{a b}\right)=\sum_{c=1}^{N} t_{a c} \otimes t_{c b}
$$

There exist the following left actions $\mathcal{R}, \mathcal{L}: \mathrm{U}_{q}\left(\mathfrak{g l}_{N}\right) \otimes \mathcal{M}_{N} \longrightarrow \mathcal{M}_{N}$ of $\mathrm{U}_{q}\left(\mathfrak{g l}_{N}\right)$ on $\mathcal{M}_{N}$ respectively defined by

$$
\mathcal{R}: x \otimes f \mapsto \sum_{(f)} f_{(1)}\left\langle f_{(2)}, x\right\rangle, \quad \mathcal{L}: x \otimes f \mapsto \sum_{(f)}\left\langle f_{(1)}, S(x)\right\rangle f_{(2)},
$$

where Sweedler's notation $\Delta(f)=\sum_{(f)} f_{(1)} \otimes f_{(2)}$ is used for the co-multiplication of $f \in \mathcal{M}_{N}$. We refer to $\mathcal{L}$ and $\mathcal{R}$ as left and right translation respectively. It is obvious but important to observe that the two actions commute. Both actions preserve the algebraic structure of $\mathcal{M}_{N}$, that is, $\mathcal{M}_{N}$ is a $\mathrm{U}_{q}\left(\mathfrak{g l}_{N}\right)$-module algebra with respect to both the $\mathcal{L}$ and $\mathcal{R}$ actions. However, note that while the $\mathcal{R}\left(\mathrm{U}_{q}\left(\mathfrak{g l}_{N}\right)\right)$-module algebra structure is defined with respect to the co-multiplication $\Delta$, the $\mathcal{L}\left(\mathrm{U}_{q}\left(\mathfrak{g l}_{N}\right)\right)$-module algebra structure is defined with respect to the opposite co-multiplication $\Delta^{\prime}$.

Denote by $L_{\lambda}^{(N)}$ the irreducible $\mathrm{U}_{q}\left(\mathfrak{g l}_{N}\right)$-module with highest weight $\lambda$. The tensor powers of $V^{(N)}$ decompose into a direct sums of simple modules with highest weight corresponding to partitions of length less than or equal to $N$, and every $L_{\lambda}^{(N)}$ with $\lambda$ such a partition appears in some tensor power of $V^{(N)}$ as a submodule. It follows that we have the following multiplicity free decomposition of $\mathcal{M}_{N}$, which may be thought as part of a quantum Peter-Weyl theorem.

Theorem 6.1. [33] As a $\mathcal{L}\left(\mathrm{U}_{q}\left(\mathfrak{g l}_{N}\right)\right) \otimes \mathcal{R}\left(\mathrm{U}_{q}\left(\mathfrak{g l}_{N}\right)\right)$-module,

$$
\mathcal{M}_{N} \cong \bigoplus_{\lambda}\left(L_{\lambda}^{(N)}\right)^{*} \otimes L_{\lambda}^{(N)}
$$

where the sum is over all partitions of lengths $\leq N$.

Given any order on the pairs $(a, b)$ with $a, b \in[1, N]$, one observes by inspection of the relations (6.1) that the ordered monomials in $t_{a b}$ span $\mathcal{M}_{N}$. Since $\operatorname{dim} L_{\lambda}^{(N)}$ is just the dimension of the irreducible $\mathfrak{g l}_{N}$-module with highest weight $\lambda, \sum_{|\lambda|=j}\left(\operatorname{dim} L_{\lambda}^{(N)}\right)^{2}=\left[\begin{array}{c}N^{2}+j-1 \\ j\end{array}\right]$, where $|\lambda|$ is the sum of the parts of $\lambda$. The right side is equal to the number of ordered monomials of degree $j$ in the $t_{a b}$. It follows that the ordered monomials are linearly independent, and hence form a basis of $\mathcal{M}_{N}$, as is well known. 
Let $\tau$ be the $\mathcal{K}$-linear algebra anti-automorphism of $\mathrm{U}_{q}\left(\mathfrak{g l}_{N}\right)$ defined by

$$
\tau\left(e_{a}\right)=f_{a}, \quad \tau\left(e_{a}\right)=f_{a}, \quad \tau\left(K_{b}\right)=K_{b}, \quad \forall a, b .
$$

Then the map $\tilde{\mathcal{L}}: \mathrm{U}_{q}\left(\mathfrak{g l}_{N}\right) \otimes \mathcal{M}_{N} \longrightarrow \mathcal{M}_{N}$ defined by $x \otimes f \mapsto \sum_{(f)}\left\langle f_{(1)}, \tau(x)\right\rangle f_{(2)}$ gives rise to another action of $\mathrm{U}_{q}\left(\mathfrak{g l}_{N}\right)$ on $\mathcal{M}_{N}$, and $\mathcal{M}_{N}$ is a module algebra over $\mathrm{U}_{q}\left(\mathfrak{g l}_{N}\right)$ under this action.

For a fixed positive integer $t \leq N$, the elements $K_{j}^{ \pm 1}, e_{i}$ and $f_{i}$ with $1 \leq j \leq t$ and $1 \leq i<t$ generate a Hopf subalgebra $\mathrm{U}_{q}\left(\mathfrak{g l}_{t}\right)$ of $\mathrm{U}_{q}\left(\mathfrak{g l}_{N}\right)$. Let $\Upsilon_{t}$ be the subalgebra of $\mathrm{U}_{q}\left(\mathfrak{g l}_{N}\right)$ generated by $K_{a}$ with $t+1 \leq a \leq N$. Then $\Upsilon_{t}$ commutes with the subalgebra $\mathrm{U}_{q}\left(\mathfrak{g l}_{t}\right)$. For each pair of positive integers $t, s \leq N$, we define the following subalgebra of $\mathcal{M}_{N}$ :

$$
\mathcal{M}_{s, t}=\left(\mathcal{M}_{N}\right)^{\mathcal{L}\left(\Upsilon_{s}\right) \otimes \mathcal{R}\left(\Upsilon_{t}\right)} .
$$

Lemma 6.2. The subalgebra $\mathcal{M}_{s, t}$ of $\mathcal{M}_{N}$ is generated by the elements $t_{i a}$ with $1 \leq i \leq s$ and $1 \leq a \leq t$, which satisfy the following relations:

$$
\begin{aligned}
t_{j b} t_{i a} & =\sum_{a^{\prime}, b^{\prime}=1}^{t} t_{i b^{\prime}} t_{j a^{\prime}} R_{a^{\prime} b, b^{\prime} a}, \quad i<j \leq s, \\
q t_{i a} t_{i b} & =\sum_{a^{\prime}, b^{\prime}=1}^{t} t_{i b^{\prime}} t_{i a^{\prime}} R_{a^{\prime} a, b^{\prime} b},
\end{aligned}
$$

Furthermore, $\mathcal{M}_{s, t}$ has a basis consisting of ordered monomials in these elements.

Proof. Let $\prod_{s}=\prod_{a=s+1}^{N} K_{a}$ and $\prod_{t}=\prod_{a=t+1}^{N} K_{a}$. Then $f$ belongs to $\mathcal{M}_{s, t}$ if and only if $\mathcal{L}_{\prod_{s}}(f)=\mathcal{R}_{\prod_{t}}(f)=f$. Since

$$
\mathcal{L}_{\prod_{S}}\left(t_{a b}\right)=\left\{\begin{array}{ll}
t_{a b}, & a \leq s, \\
q^{-1} t_{a b}, & a>s,
\end{array} \quad \mathcal{R}_{\prod_{t}}\left(t_{a b}\right)= \begin{cases}t_{a b}, & b \leq t, \\
q t_{a b}, & b>t,\end{cases}\right.
$$

we have $t_{a_{1} b_{1}} t_{a_{2} b_{2}} \cdots t_{a_{k} b_{k}} \in \mathcal{M}_{s, t}$ if and only if $1 \leq a_{i} \leq s$ and $1 \leq b \leq t$ for all $i$. Such elements obviously form a basis of $\mathcal{M}_{s, t}$. This shows that the subalgebra $\mathcal{M}_{s, t}$ is generated by the elements $t_{i j}$ with $1 \leq i \leq s$ and $1 \leq j \leq t$.

The relations (6.6) follow from (6.1) and the explicit form of the $R$-matrix (6.2).

Remark 6.3. Let $V^{(t)}$ be the natural module for $\mathrm{U}_{q}\left(\mathfrak{g l}_{t}\right)$ and let $S_{q}\left(V^{(t)}\right)$ be the braided symmetric algebra over $V^{(t)}$. Consider the algebra $S_{q}\left(V^{(t)}\right)^{\otimes s}$ discussed above. The relations (6.6) show that this algebra is isomorphic to $\mathcal{M}_{s, t}$, as was pointed out in [5].

The next result is the quantum Howe duality (cf. [33]) applied to the subalgebra $\mathcal{M}_{s, t}$ of $\mathcal{M}_{N}$.

Theorem 6.4. (1) The subalgebra $\mathcal{M}_{s, t}$ of $\mathcal{M}_{N}$ is a $\mathcal{L}\left(\mathrm{U}_{q}\left(\mathfrak{g l}_{s}\right)\right) \otimes \mathcal{R}\left(\mathrm{U}_{q}\left(\mathfrak{g l}_{t}\right)\right)$ module algebra, and

$$
\mathcal{M}_{s, t} \cong \bigoplus_{\lambda}\left(L_{\lambda}^{(s)}\right)^{*} \otimes L_{\lambda}^{(t)}
$$

where the summation is over all partitions of length $\leq \min (s, t)$. 
(2) The subalgebra $\mathcal{M}_{s, t}$ is a module algebra over $\tilde{\mathcal{L}}\left(\mathrm{U}_{q}\left(\mathfrak{g l}_{s}\right)\right) \otimes \mathcal{R}\left(\mathrm{U}_{q}\left(\mathfrak{g l}_{t}\right)\right)$ and

$$
\mathcal{M}_{s, t} \cong \bigoplus_{\lambda} L_{\lambda}^{(s)} \otimes L_{\lambda}^{(t)} \quad \text { as } \tilde{\mathcal{L}}\left(\mathrm{U}_{q}\left(\mathfrak{g l}_{s}\right)\right) \otimes \mathcal{R}\left(\mathrm{U}_{q}\left(\mathfrak{g l}_{t}\right)\right) \text {-module }
$$

where the range of the summation in $\lambda$ is the same as in part (1).

Proof. Consider the following assertions, which we shall prove shortly. For all partitions $\lambda$,

$$
\begin{aligned}
& \left(L_{\lambda}^{(N)}\right)^{\Pi_{t}}= \begin{cases}L_{\lambda}^{(t)}, & \text { if the length of } \lambda \text { is } \leq t, \\
0, & \text { otherwise, }\end{cases} \\
& \left(\left(L_{\lambda}^{(N)}\right)^{*}\right)^{\prod_{s}}= \begin{cases}\left(L_{\lambda}^{(s)}\right)^{*}, & \text { if the length of } \lambda \text { is } \leq s, \\
0, & \text { otherwise. }\end{cases}
\end{aligned}
$$

Granted (6.9), part (11) of the theorem follows immediately. Part (2) then follows from the second statement in part (1).

We turn to the proof of equation (6.9), for which we provide details, since similar arguments will be used later. Consider the subalgebras $\mathrm{U}_{q}\left(\mathfrak{l}_{t}\right)$ and $\mathrm{U}_{q}\left(\mathfrak{p}_{t}\right)$ of $\mathrm{U}_{q}\left(\mathfrak{g l}_{N}\right)$, defined as follows.

- $\mathrm{U}_{q}\left(\mathfrak{l}_{t}\right)$ is generated by $K_{a}^{ \pm 1}$ (for all $\left.a\right)$ and $e_{b}, f_{b}(b \neq t)$;

- $\mathrm{U}_{q}\left(\mathfrak{p}_{t}\right)$ is generated by the elements of $\mathrm{U}_{q}\left(\mathfrak{l}_{t}\right)$ and $e_{t}$.

The irreducible $\mathrm{U}_{q}\left(\mathfrak{g l}_{N}\right)$-module $L_{\lambda}^{(N)}$ with highest weight $\lambda=\left(\lambda_{1}, \lambda_{2}, \ldots, \lambda_{N}\right)$ may be constructed as follows. Let $L_{\lambda}^{(N), 0}$ be the irreducible $\mathrm{U}_{q}\left(\mathfrak{p}_{t}\right)$-module with highest weight $\lambda$, and construct the generalised Verma module $\mathcal{V}_{\lambda}=\mathrm{U}_{q}\left(\mathfrak{g l}_{N}\right) \otimes_{\mathrm{U}_{q}\left(\mathfrak{p}_{t}\right)} L_{\lambda}^{(N), 0}$. Then the quotient of $\mathcal{V}_{\lambda}$ by its unique maximal submodule is isomorphic to $L_{\lambda}^{(N)}$.

If $\mu$ is a weight of the generalised Verma module but not of $L_{\lambda}^{(N), 0}$, then it follows from the definition of $\mathcal{V}_{\lambda}$ that $\mu=\lambda-\sum_{i<t<\beta} k_{i \beta}\left(\epsilon_{i}-\epsilon_{\beta}\right)$ for some nonnegative integers $k_{i \beta}$ which are not all nonzero. Assume that $\lambda \in \mathbb{Z}_{+}^{N}$. Then $\left(\mathcal{V}_{\lambda}\right)^{\prod_{t}} \neq 0$ if and only if $\lambda_{t+1}=\lambda_{t+2}=\cdots=\lambda_{N}=0$ and if this holds, $\left(\mathcal{V}_{\lambda}\right)^{\prod_{t}}=L_{\lambda}^{(N), 0}$. It follows that $\left(L_{\lambda}^{(N)}\right)^{\prod_{t}}=L_{\lambda}^{(N), 0}$. Note that $L_{\lambda}^{(N), 0}$ is an irreducible $\mathrm{U}_{q}\left(\mathfrak{l}_{t}\right)$-module and $e_{t}$ acts by 0 . It restricts to the irreducible module over $\mathrm{U}_{q}\left(\mathfrak{g l}_{t}\right)$ with highest weight $\left(\lambda_{1}, \ldots, \lambda_{t}\right)$, of which it is the inflation to $\mathrm{U}_{q}\left(\mathfrak{p}_{t}\right)$.

Now consider the subalgebras $\mathrm{U}_{q}\left(\mathfrak{l}_{s}\right)$ and $\mathrm{U}_{q}\left(\overline{\mathfrak{p}}_{s}\right)$ of $\mathrm{U}_{q}\left(\mathfrak{g l}_{N}\right)$ defined by

- $\mathrm{U}_{q}\left(\mathfrak{l}_{s}\right)$ is generated by $K_{a}^{ \pm 1}$ (for all $\left.a\right)$ and $e_{b}, f_{b}(b \neq s)$;

- $\mathrm{U}_{q}\left(\overline{\mathfrak{p}}_{s}\right)$ is generated by the elements of $\mathrm{U}_{q}\left(\mathfrak{l}_{s}\right)$ and $f_{s}$.

Let $\Gamma_{-\lambda}^{(N), 0}$ be the irreducible $\mathrm{U}_{q}\left(\mathfrak{p}_{t}\right)$-module with lowest weight $-\lambda$, and $\overline{\mathcal{V}}_{-\lambda}=$ $\mathrm{U}_{q}\left(\mathfrak{g l}_{N}\right) \otimes_{\mathrm{U}_{q}\left(\overline{\mathfrak{p}}_{s}\right)} \Gamma_{-\lambda}^{(N), 0}$ the corresponding generalised Verma module. Then the quotient of $\overline{\mathcal{V}}_{-\lambda}$ by its unique maximal submodule is the irreducible $\mathrm{U}_{q}\left(\mathfrak{g l}_{N}\right)$-module $\Gamma_{-\lambda}^{(N)}$ with lowest weight $-\lambda$; that is, $\Gamma_{-\lambda}^{(N)}=\left(L_{\lambda}^{(N)}\right)^{*}$.

Now if $\nu$ is a weight of $\overline{\mathcal{V}}_{-\lambda}$ but not of $\Gamma_{-\lambda}^{(N), 0}$, then $\nu=-\lambda+\sum_{i \leq s<\beta} k_{i \beta}\left(\epsilon_{i}-\epsilon_{\beta}\right)$ for some nonnegative integers $k_{i \beta}$ which are not all nonzero. Assume $\lambda \in \mathbb{Z}_{+}^{N}$ and is dominant. Then arguing as above one sees that $\left(\Gamma_{-\lambda}^{(N)}\right)^{\mathcal{L}_{\Pi_{s}}} \cong\left(\overline{\mathcal{V}}_{-\lambda}\right)^{\mathcal{L}_{\Pi_{s}}}=\Gamma_{-\lambda}^{(N), 0}$ 
if $\lambda=\left(\lambda_{1}, \ldots, \lambda_{s}, 0, \ldots, 0\right)$ and $\left(\Gamma_{-\lambda}^{(N)}\right)^{\mathcal{L}_{\Pi_{s}}}=0$ otherwise. In the former case, $\Gamma_{-\lambda}^{(N), 0}$ restricts to the irreducible $\mathrm{U}_{q}\left(\mathfrak{g l}_{s}\right)$-module with lowest weight $-\left(\lambda_{1}, \ldots, \lambda_{s}\right)$, that is, to $\left(L_{\left(\lambda_{1}, \ldots, \lambda_{s}\right)}^{(s)}\right)^{*}$.

This completes the proof of Theorem 6.4.

Let $\bar{t}_{a b} \in \mathrm{U}_{q}\left(\mathfrak{g l}_{N}\right)^{*}(a, b \in[1, N])$ be defined by

$$
\left\langle\bar{t}_{a b}, x\right\rangle=\left\langle t_{b a}, S(x)\right\rangle, \quad \forall x \in \mathrm{U}_{q}\left(\mathfrak{g l}_{N}\right) .
$$

These are the coefficient functions of the dual $\bar{\pi}$ of the natural representation $\pi$ of $\mathrm{U}_{q}\left(\mathfrak{g l}_{N}\right)$ in $V^{*}$. The subalgebra $\overline{\mathcal{M}}_{N}$ of $\mathrm{U}_{q}\left(\mathfrak{g l}_{N}\right)^{*}$ generated by these elements is also a bi-algebra with comultiplication defined by

$$
\Delta\left(\bar{t}_{a b}\right)=\sum_{c=1}^{N} \bar{t}_{a c} \otimes \bar{t}_{c b} .
$$

There exist left actions

$$
\mathcal{L}, \mathcal{R}: \mathrm{U}_{q}\left(\mathfrak{g l}_{N}\right) \otimes \overline{\mathcal{M}}_{N} \longrightarrow \overline{\mathcal{M}}_{N}
$$

defined in the same way as (6.3), and with respect to each of these actions, $\overline{\mathcal{M}}_{N}$ is a $\mathrm{U}_{q}\left(\mathfrak{g l}_{N}\right)$-module algebra.

The elements $t_{a b}$ and $\bar{t}_{a b}(a, b \in[1, N])$ together generate a subalgebra $\mathcal{K}\left[G L_{q}(N)\right]$ of the $\mathrm{U}_{q}\left(\mathfrak{g l}_{N}\right)^{*}$, which is clearly a bi-algebra. The following relations hold in $\mathcal{K}\left[G L_{q}(N)\right]$

$$
\sum_{c=1}^{N} t_{a c} \bar{t}_{b c}=\sum_{c=1}^{N} \bar{t}_{a c} t_{b c}=\delta_{a b} .
$$

It follows that $\mathcal{K}\left[G L_{q}(N)\right]$ has the structure of a Hopf algebra with antipode given by

$$
S\left(t_{a b}\right)=\bar{t}_{b a}, \quad S\left(\bar{t}_{b a}\right)=q^{2(a-b)} t_{a b}
$$

This Hopf algebra could be thought as the algebra of regular functions on a quantum analogue of the general linear group.

For each pair of positive integers $t, s \leq N$, define the following subalgebra of $\overline{\mathcal{M}}_{N}$ (cf. (6.5)):

$$
\overline{\mathcal{M}}_{s, t}=\left(\overline{\mathcal{M}}_{N}\right)^{\mathcal{L}\left(\Upsilon_{s}\right) \otimes \mathcal{R}\left(\Upsilon_{t}\right)} .
$$

Note that $\overline{\mathcal{M}}_{s, t}=\overline{\mathcal{M}}_{N}$ if $s=t=N$. Using the fact that $\bar{t}_{a b}=S\left(t_{b a}\right)$ we deduce from (6.1) the following result.

Lemma 6.5. The subalgebra $\overline{\mathcal{M}}_{s, t}$ is generated by the elements $\bar{t}_{i \alpha}$ with $1 \leq i \leq s$ and $1 \leq a \leq t$, which satisfy the following relations:

$$
\begin{aligned}
& \bar{t}_{j b} \bar{t}_{i a}=\sum_{a^{\prime}, b^{\prime}=1}^{t} \bar{t}_{i a^{\prime}} \bar{t}_{j b^{\prime}} R_{a a^{\prime}, b b^{\prime}}^{-1}, \quad i<j \leq s, \\
& q^{-1} \bar{t}_{i b} \bar{t}_{i a}=\sum_{a^{\prime}, b^{\prime}=1}^{t} \bar{t}_{i a^{\prime}} \bar{t}_{i b^{\prime}} R_{a a^{\prime}, b b^{\prime}}^{-1} .
\end{aligned}
$$

Furthermore, $\overline{\mathcal{M}_{s, t}}$ has a basis consisting of ordered monomials in these elements. 
Denote by $\bar{V}^{(t)}$ the dual of the natural module for $\mathrm{U}_{q}\left(\mathfrak{g l}_{t}\right)$, and let $S_{q}\left(\bar{V}^{(t)}\right)$ be the braided symmetric algebra over $\bar{V}^{(t)}$. Define algebraic structures on tensor powers of $\bar{S}_{q}:=S_{q}\left(\bar{V}^{(t)}\right)$ recursively by

$$
\mu_{k+1}:=\mu_{k} \otimes P R^{-T} \otimes \mu_{1}: \bar{S}_{q}^{\otimes k} \otimes \bar{S}_{q} \longrightarrow \bar{S}_{q}^{\otimes k} \otimes \bar{S}_{q}
$$

where $\mu_{1}$ denotes the multiplication of $\bar{S}_{q}$, and $R^{-T}=\sum_{s} \bar{\beta}_{s} \otimes \bar{\alpha}_{s}$ if we write the inverse of the R-matrix as $R^{-1}=\sum_{s} \bar{\alpha}_{s} \otimes \bar{\beta}_{s}$. It is easy to show that $R^{-T}$ also satisfies all the relations (2.1) and (2.2). Thus Theorem 2.3 and Lemma 2.5 are valid when $R$ is replaced by $R^{-T}$. It follows that $\left(S_{q}\left(\bar{V}^{(t)}\right)^{\otimes k}, \mu_{k}\right)$ has the structure of a module algebra for $\mathrm{U}_{q}\left(\mathfrak{g l}_{t}\right)$ for any $k$.

Corollary 6.6. (1) For any fixed $i$, the subalgebra of $\overline{\mathcal{M}}_{s, t}$ generated by the elements $\bar{t}_{i a}(1 \leq a \leq t)$ is isomorphic to $S_{q}\left(\bar{V}^{(t)}\right)$.

(2) As a $\mathrm{U}_{q}\left(\mathfrak{g l}_{t}\right)$-module algebra, $\overline{\mathcal{M}}_{s, t}$ is isomorphic to $S_{q}\left(\bar{V}^{(t)}\right)^{\otimes s}$ with multiplication $\mu_{s}$.

Proof. The second relation in (6.11) implies (1), while the first relation implies (2).

The following result is now clear.

Theorem 6.7. The subalgebra $\overline{\mathcal{M}}_{s, t}$ is generated by the elements $\bar{t}_{i j}$ with $1 \leq i \leq s$ and $1 \leq j \leq t$, and has a basis consisting of ordered monomials in these elements. Furthermore, $\overline{\mathcal{M}}_{s, t}$ is a $\mathrm{U}_{q}\left(\mathfrak{g l}_{s}\right) \otimes \mathrm{U}_{q}\left(\mathfrak{g l}_{t}\right)$-module algebra via the action $\mathcal{L} \otimes \mathcal{R}$ and has module decomposition

$$
\overline{\mathcal{M}}_{s, t} \cong \bigoplus_{\lambda} L_{\lambda}^{(s)} \otimes\left(L_{\lambda}^{(t)}\right)^{*}
$$

where the summation is over all partitions of length $\leq \min (s, t)$.

6.2. Invariant theory for the quantum general linear group. Fix positive integers $n, k, l \leq N$. We shall work with the following $\mathrm{U}_{q}\left(\mathfrak{g l}_{n}\right)$-module algebra.

Definition 6.8. Recall that we have $\mathcal{R}\left(\mathrm{U}_{q}\left(\mathfrak{g l}_{n}\right)\right)$-module algebras $\mathcal{M}_{k, n}$ and $\overline{\mathcal{M}}_{l, n}$, respectively defined in (6.5) and (6.10). If we define multiplication in $\mathcal{A}_{k, l}:=\mathcal{M}_{k, n} \otimes$ $\overline{\mathcal{M}}_{l, n}$ by (2.4) (cf. Theorem 2.3) $\mathcal{A}_{k, l}$ becomes a $\mathrm{U}_{q}\left(\mathfrak{g l}_{n}\right)$-module algebra, with action defined for any $f \otimes g \in \mathcal{A}_{k, l}$ and $x \in \mathrm{U}_{q}\left(\mathfrak{g l}_{n}\right)$ by

$$
x(f \otimes g)=\sum_{(x)} \mathcal{R}_{x_{(1)}}(f) \otimes \mathcal{R}_{x_{(2)}}(g) .
$$

We investigate the structure of $\mathcal{A}_{k, l}$. Let $X_{i a}:=t_{i a} \otimes 1$ and $Y_{\beta b}:=1 \otimes \bar{t}_{\beta b}$ with $i \in[1, k], a, b \in[1, n]$ and $\beta \in[1, l]$. Then we have

$$
Y_{b \beta} X_{i a}=\sum_{a^{\prime}, b^{\prime}=1}^{n}\left(R^{-T}\right)_{a^{\prime} b, b^{\prime} a} X_{i b^{\prime}} Y_{a^{\prime} \beta},
$$


where $R^{-T}=1 \otimes 1+\left(q^{-1}-1\right) \sum_{a=1}^{n} E_{a a} \otimes E_{a a}-\left(q-q^{-1}\right) \sum_{1 \leq a<b \leq n} E_{b a} \otimes E_{a b}$. These relations can be made more explicit, as follows.

$$
\begin{aligned}
& Y_{\beta a} X_{i a}=q X_{i a} Y_{\beta a}-\left(q-q^{-1}\right) \sum_{c=a}^{n} X_{i c} Y_{\beta c}, \\
& Y_{\beta b} X_{i a}=X_{i a} Y_{\beta b}, \quad \text { if } a \neq b .
\end{aligned}
$$

Our purpose is to study the subalgebra of $\mathcal{A}_{k, l}$ comprising its $\mathrm{U}_{q}\left(\mathfrak{g l}_{n}\right)$-invariant elements:

$$
\left(\mathcal{A}_{k, l}\right)^{\mathrm{U}_{q}\left(\mathfrak{g l}_{n}\right)}=\left\{f \in \mathcal{A}_{k, l} \mid x(f)=\epsilon(x) f, \forall x \in \mathrm{U}_{q}\left(\mathfrak{g l}_{n}\right)\right\} .
$$

Lemma 6.9. Let $\Psi_{i \beta}:=\sum_{a=1}^{n} X_{i a} Y_{\beta a}$ for $1 \leq i \leq k$ and $1 \leq \beta \leq l$.

(1) The elements $\Psi_{i \beta}(1 \leq i \leq k, 1 \leq \beta \leq l)$ belong to $\left(\mathcal{A}_{k, l}\right)^{\mathrm{U}_{q}\left(\mathfrak{g l}_{n}\right)}$, the invariant subalgebra of $\mathcal{A}_{k, l}$.

(2) The following relations hold in $\mathcal{A}_{k, l}$ among the elements $\Psi_{i \beta}, X_{i a}$ and $Y_{\alpha b}$.

$$
\begin{array}{ll}
\Psi_{j \beta} X_{i a}=X_{i a} \Psi_{j \beta}, & i<j, \\
X_{j a} \Psi_{i \beta}-\Psi_{i \beta} X_{j a}=\left(q-q^{-1}\right) X_{i a} \Psi_{j \beta}, & i<j, \\
\Psi_{i \beta} X_{i a}=q^{-1} X_{i a} \Psi_{i \beta}, & \\
\Psi_{j \beta} Y_{\alpha b}=Y_{\alpha b} \Psi_{j \beta}, & \alpha<\beta, \\
\Psi_{j \alpha} Y_{\beta b}-Y_{\beta b} \Psi_{j \alpha}=\left(q-q^{-1}\right) Y_{\alpha b} \Psi_{j \beta}, & \alpha<\beta, \\
\Psi_{i \beta} Y_{\beta b}=q Y_{\beta b} \Psi_{i \beta} . &
\end{array}
$$

(3) The elements $\Psi_{i \beta}$ satisfy the following relations.

$$
\begin{array}{ll}
\Psi_{j \beta} \Psi_{i \alpha}=\Psi_{i \alpha} \Psi_{j \beta}, & i<j, \alpha<\beta, \\
\Psi_{j \alpha} \Psi_{i \beta}-\Psi_{i \beta} \Psi_{j \alpha}=\left(q-q^{-1}\right) \Psi_{i \alpha} \Psi_{j \beta}, & i<j, \quad \alpha<\beta, \\
\Psi_{i \beta} \Psi_{i \alpha}=q^{-1} \Psi_{i \alpha} \Psi_{i \beta}, & \alpha<\beta, \\
\Psi_{j \beta} \Psi_{i \beta}=q \Psi_{i \beta} \Psi_{j \beta}, & i<j .
\end{array}
$$

Proof. Part (1) is a special case of (2.12), but we provide an explicit computation. Let $\pi$ be the natural representation of $\mathrm{U}_{q}\left(\mathfrak{g l}_{n}\right)$ and $\bar{\pi}$ its dual. For all $x \in \mathrm{U}_{q}\left(\mathfrak{g l}_{n}\right)$, we have

$$
x \Psi_{i \alpha}=\sum_{a, a^{\prime} b^{\prime}=1}^{n} \sum_{(x)} X_{i a^{\prime}} Y_{\alpha b^{\prime}} \pi\left(x_{(1)}\right)_{a^{\prime} a} \bar{\pi}\left(x_{(2)}\right)_{b^{\prime} a}=\epsilon(x) \Psi_{i \alpha} .
$$

This proves part (1).

All the relations in part (2) are proved in a similar way by routine calculations. We illustrate the proof by considering the second last relation. If $\alpha<\beta$, then $\Psi_{j \alpha} Y_{\beta b}=\sum_{a, a^{\prime}, b^{\prime}=1}^{n} X_{j a} R_{a a^{\prime}, b b^{\prime}} Y_{\beta b^{\prime}} Y_{\alpha a^{\prime}}$, where $R_{a a^{\prime}, b b^{\prime}}$ are the entries of the $R$-matrix acting on the tensor square of the natural representation of $\mathrm{U}_{q}\left(\mathfrak{g l}_{n}\right)$. Let $P$ denote the permutation map on this space. Then the above relation may be written

$$
\Psi_{j \alpha} Y_{\beta b}=\sum_{a, a^{\prime}, b^{\prime}=1}^{n} X_{j a}(\check{R} P)_{b b^{\prime}, a a^{\prime}} Y_{\beta b^{\prime}} Y_{\alpha a^{\prime}}
$$


We also have, similarly,

$$
Y_{\beta b} \Psi_{j \alpha}=\sum_{a, a^{\prime}, b^{\prime}=1}^{n} X_{j a}\left(R^{-1}\right)_{b b^{\prime}, a a^{\prime}} Y_{\beta b^{\prime}} Y_{\alpha a^{\prime}}
$$

Using the skein relation $\check{R}-\check{R}^{-1}=q-q^{-1}$, where $\check{R}=P R$, we obtain

$$
\begin{aligned}
\Psi_{j \alpha} Y_{\beta b}-Y_{\beta b} \Psi_{j \alpha} & =\left(q-q^{-1}\right) \sum_{a, a^{\prime}, b^{\prime}=1}^{n} X_{j a} P_{b b^{\prime}, a a^{\prime}} Y_{\beta b^{\prime}} Y_{\alpha a^{\prime}} \\
& =\left(q-q^{-1}\right) \Psi_{j \beta} Y_{\alpha b},
\end{aligned}
$$

which proves the second last relation of part (2) in view of the fourth relation.

Part (3) is a straightforward consequence of part (2).

We shall prove the following result, which is the quantum analogue of the first fundamental theorem of invariant theory for the quantum general linear group.

Theorem 6.10. The invariant subalgebra $\left(\mathcal{A}_{k, l}\right)^{\mathrm{U}_{q}\left(\mathfrak{g l}_{n}\right)}$ is generated by the elements $\Psi_{i \beta}$ with $1 \leq i \leq k, 1 \leq \beta \leq l$.

The proof will be given in the next section.

6.3. Proof of the FFT for the quantum general linear group. This section is devoted to the proof of Theorem 6.10. The techniques we use are similar to those in [10]. However, the object corresponding to $\mathcal{A}_{k, l}$ in [10] is not a $\mathrm{U}_{q}\left(\mathfrak{g l}_{n}\right)$-module algebra, so that our concept of being 'generated by' a subset is quite different from the set up of [10].

6.3.1. The case $n \geq \max (k, l)$. We consider the algebra $\mathcal{M}_{s t}$ defined by (6.5) with $N=n, s=k$ and $t=l$. Then $\Delta\left(\mathcal{M}_{k, l}\right) \subset \mathcal{M}_{k, n} \otimes \mathcal{M}_{n, l}$ under the co-multiplication on $\mathcal{M}_{n}$, as can be seen from Theorem 6.4(1) and the formula $\Delta\left(t_{i \beta}\right)=\sum_{a=1}^{n} t_{i a} \otimes t_{a \beta}$, for all $i \in[1, k]$ and $\beta \in[1, l]$. It follows that $(\mathrm{id} \otimes S) \Delta\left(\mathcal{M}_{k, l}\right) \subset \mathcal{A}_{k, l}$.

Lemma 6.11. The $\mathcal{K}$-linear map

$$
\Delta_{k, l}: \mathcal{M}_{k, l} \longrightarrow \mathcal{A}_{k, l}
$$

given by $\Delta_{k, l}(f):=(i d \otimes S) \Delta(f)$, is injective and satisfies $\Delta_{k, l}\left(\mathcal{M}_{k, l}\right) \subseteq\left(\mathcal{A}_{k, l}\right)^{\mathrm{U}_{q}\left(\mathfrak{g l}_{n}\right)}$.

Proof. To prove injectivity of $\Delta_{k, l}$, we note that for all $f \in \operatorname{ker} \Delta_{k, l}$,

$$
(\epsilon \otimes \mathrm{id}) \Delta_{k, l}(f)=\sum_{(f)} 1 \otimes \epsilon\left(f_{(1)}\right) S\left(f_{(2)}\right)=S(f)=0 .
$$

Thus ker $\Delta_{k, l}=0$ since $S$ is invertible.

Now for $x \in \mathrm{U}_{q}\left(\mathfrak{g l}_{n}\right)$ and $f \in \mathcal{M}_{k, l}$, we have

$$
\begin{aligned}
x \Delta_{k, l}(f) & =\sum_{(f),(x)} \mathcal{R}_{x_{(1)}}\left(f_{(1)}\right) \otimes \mathcal{R}_{x_{(2)}}\left(S\left(f_{(2)}\right)\right) \\
& =\sum_{(f),(x)} f_{(1)}\left\langle f_{(2)}, x_{(1)}\right\rangle \otimes\left\langle f_{(2)}, S\left(x_{(2)}\right\rangle S\left(f_{(3)}\right)\right. \\
& =\epsilon(x) \sum_{(f),(x)} f_{(1)} \otimes S\left(f_{(2)}\right) .
\end{aligned}
$$


This proves the second claim of the lemma.

It is useful to note that $\Delta_{k, l}\left(t_{i \beta}\right)=\Psi_{i \beta}$.

Lemma 6.12. If $n \geq \max (k, l)$, then $\left(\mathcal{A}_{k, l}\right)^{\mathrm{U}_{q}\left(\mathfrak{g l}_{n}\right)}=\Delta_{k, l}\left(\mathcal{M}_{k, l}\right)$.

Proof. Using parts (11) and (2) of Theorem 6.4 and also Theorem 6.7, we can show that as a module over $\tilde{\mathcal{L}}\left(\mathrm{U}_{q}\left(\mathfrak{g l}_{k}\right)\right) \otimes \mathcal{R}\left(\mathrm{U}_{q}\left(\mathfrak{g l}_{n}\right)\right) \otimes \mathcal{L}\left(\mathrm{U}_{q}\left(\mathfrak{g l}_{l}\right)\right) \otimes \mathcal{R}\left(\mathrm{U}_{q}\left(\mathfrak{g l}_{n}\right)\right)$,

$$
\mathcal{A}_{k, l}=\bigoplus_{\lambda, \mu} L_{\lambda}^{(k)} \otimes L_{\lambda}^{(n)} \otimes L_{\mu}^{(l)} \otimes\left(L_{\mu}^{(n)}\right)^{*}
$$

where the summation is over partitions $\lambda, \mu$ where $\lambda$ has length $\leq k$ and $\mu$ has length $\leq l$. Since $n \geq \max (k, l)$, it follows that

$$
\left(\mathcal{A}_{k, l}\right)^{\mathrm{U}_{q}\left(\mathfrak{g l}_{n}\right)}=\bigoplus_{\lambda} L_{\lambda}^{(k)} \otimes L_{\lambda}^{(l)}
$$

as a module for $\tilde{\mathcal{L}}\left(\mathrm{U}_{q}\left(\mathfrak{g l}_{k}\right)\right) \otimes \mathcal{L}\left(\mathrm{U}_{q}\left(\mathfrak{g l}_{l}\right)\right)$, where the summation is over all partitions of length $\leq \min (k, l)$. Thus by Theorem 6.4(2), $\mathcal{M}_{k, l} \cong\left(\mathcal{A}_{k, l}\right)^{\mathrm{U}_{q}\left(\mathfrak{g l}_{n}\right)}$ as $\tilde{\mathcal{L}}\left(\mathrm{U}_{q}\left(\mathfrak{g l}_{k}\right)\right) \otimes$ $\mathcal{R}\left(\mathrm{U}_{q}\left(\mathfrak{g l}_{l}\right)\right)$-module. The lemma now follows from the injectivity of $\Delta_{k, l}$, proved in Lemma 6.11.

In the next Lemma, we use the fact that the elements of $\mathcal{M}_{k, l}$ are linear functions on $\mathrm{U}_{q}\left(\mathfrak{g l}_{n}\right)$.

Lemma 6.13. Assume that $n \geq \max (k, l)$.

(1) There exists the $\mathbb{Z}_{+} \times \mathbb{Z}_{+}$-graded vector space bijection

$$
\begin{aligned}
& \varpi: \mathcal{M}_{k, l} \otimes \mathcal{M}_{k, l} \longrightarrow \mathcal{M}_{k, l} \otimes \mathcal{M}_{k, l}, \\
& f \otimes g \mapsto \sum_{(f),(g)} f_{(1)} \otimes g_{(1)}\left\langle f_{(2)} \otimes g_{(2)}, R^{-1}\right\rangle
\end{aligned}
$$

(2) Denote by $\nu$ the multiplication in $\mathcal{M}_{k, l}$. For any $f, g \in \mathcal{M}_{k, l}$, the product of $\Delta_{k, l}(f)$ and $\Delta_{k, l}(g)$ in $\mathcal{A}_{k, l}$ is given by

$$
\Delta_{k, l}(f) \Delta_{k, l}(g)=\Delta_{k, l} \circ \nu \circ \varpi(f \otimes g) .
$$

Proof. To prove part (1), we need only show that the image of $\varpi$ is in $\mathcal{M}_{k, l} \otimes \mathcal{M}_{k, l}$, since then the existence of the inverse map of $\varpi$ follows from the invertibility of $R$. Note that for all $\alpha, \beta \in[1, l]$, we have $\left\langle t_{a \alpha} \otimes t_{b \beta}, R^{-1}\right\rangle=0$ unless both $a$ and $b$ belong to $[1, l]$. This can be seen from the explicit formula for the $R$-matrix in the tensor square of the natural representation of $\mathrm{U}_{q}\left(\mathfrak{g l}_{n}\right)$. More generally, for any ordered monomials $f_{1}=t_{a_{1} \alpha_{1}} \cdots t_{a_{r} \alpha_{r}}$ and $f_{2}=t_{b_{1} \beta_{1}} \cdots t_{b_{1} \beta_{1}}$, one shows, using the defining properties of $R$ under co-multiplication that $\left\langle f_{1} \otimes f_{2}, R^{-1}\right\rangle=0$ unless all $a_{i}$ and $b_{j}$ belong to $[1, l]$. It follows from this that if $f, g \in \mathcal{M}_{k, l}$ then $\sum_{(f),(g)} f_{(1)} \otimes$ $g_{(1)}\left\langle f_{(2)} \otimes g_{(2)}, R^{-1}\right\rangle$ belongs to $\mathcal{M}_{k, l} \otimes \mathcal{M}_{k, l}$. 
Part (2) is proved as follows:

$$
\begin{aligned}
\Delta_{k, l}(f) \Delta_{k, l}(g) & =\sum_{(f),(g)} f_{(1)} g_{(1)} \otimes S\left(g_{(3)} f_{(3)}\right)\left\langle f_{(2)} \otimes g_{(2)}, R^{-1}\right\rangle \\
& =\sum_{(f),(g)} f_{(1)} g_{(1)} \otimes S\left(f_{(2)} g_{(2)}\right)\left\langle f_{(3)} \otimes g_{(3)}, R^{-1}\right\rangle \\
& =\Delta_{k, l}\left(\sum_{(f),(g)} f_{(1)} g_{(1)}\left\langle f_{(2)} \otimes g_{(2)}, R^{-1}\right\rangle\right) \\
& =\Delta_{k, l} \circ \nu \circ \varpi(f \otimes g) .
\end{aligned}
$$

This completes the proof of the lemma.

Proof of Theorem 6.10 when $n \geq \max (k, l)$. By Theorem 6.4(1), $\mathcal{M}_{k, l}$ is generated by the elements $t_{i \beta}$, and we have seen that $\Delta_{k, l}\left(t_{i \beta}\right)=\Psi_{i \beta}$. Now the degree $i$ homogeneous subspace $\left(\mathcal{M}_{k, l}\right)_{i}(i \geq 1)$ of $\mathcal{M}_{k, l}$ is equal to $\nu \circ \varpi\left(\left(\mathcal{M}_{k, l}\right)_{i-1} \otimes\left(\mathcal{M}_{k, l}\right)_{1}\right)$ by Lemma 6.13(1). Using Lemma 6.13(2), one can show by induction on the degree of $\left(\mathcal{M}_{k, l}\right)_{i}$ that every element in $\Delta_{k, l}\left(\mathcal{M}_{k, l}\right)_{i}$ can be expressed as a linear combination of products of $\Psi_{i \beta}$. Now Theorem 6.10 follows from Lemma 6.12,

6.3.2. The case $n<\max (k, l)$.

Proof of Theorem 6.10 when $n<\max (k, l)$. We shall treat separately the situations $\min (k, l) \leq n<\max (k, l)$ and $n<\min (k, l)$. In both situations, we have the following $\overline{\tilde{\mathcal{L}}}\left(\mathrm{U}_{q}\left(\mathfrak{g l}_{k}\right)\right) \otimes \mathcal{L}\left(\mathrm{U}_{q}\left(\mathfrak{g l}_{l}\right)\right)$-module decomposition of $\left(\mathcal{A}_{k, l}\right)^{\mathrm{U}_{q}\left(\mathfrak{g l}_{n}\right)}$.

$$
\left(\mathcal{A}_{k, l}\right)^{\mathrm{U}_{q}\left(\mathfrak{g l}_{n}\right)}=\bigoplus_{\lambda} L_{\lambda}^{(k)} \otimes L_{\lambda}^{(l)},
$$

where the summation is over all partitions of length $\leq m:=\min (k, l, n)$.

Let $\Upsilon_{k, m}$ be the subalgebra of $\mathrm{U}_{q}\left(\mathfrak{g l}_{k}\right)$ generated by the elements $K_{s}^{ \pm 1}$ with $m+1 \leq$ $s \leq k$, and similarly define the subalgebra $\Upsilon_{l, m}$ of $\mathrm{U}_{q}\left(\mathfrak{g l}_{l}\right)$. Using the fact that $\tilde{\mathcal{L}}\left(\mathrm{U}_{q}\left(\mathfrak{g l}_{k}\right)\right) \otimes \mathcal{L}\left(\mathrm{U}_{q}\left(\mathfrak{g l}_{l}\right)\right)$ commutes with the $\mathrm{U}_{q}\left(\mathfrak{g l}_{n}\right)$-action on $\mathcal{A}_{k, l}$ we may also define the subalgebra

$$
\mathcal{A}_{m}^{0}:=\left(\left(\mathcal{A}_{k, l}\right)^{\mathrm{U}_{q}\left(\mathfrak{g l}_{n}\right)}\right)^{\tilde{\mathcal{L}}\left(\Upsilon_{k, m}\right) \otimes \mathcal{L}\left(\Upsilon_{l, m}\right)}=\left(\left(\mathcal{A}_{k, l}\right)^{\tilde{\mathcal{L}}\left(\Upsilon_{k, m}\right) \otimes \mathcal{L}\left(\Upsilon_{l, m}\right)}\right)^{\mathrm{U}_{q}\left(\mathfrak{g l}_{n}\right)},
$$

Then $\mathcal{A}_{m}^{0}$ is generated by the elements

$$
\Psi_{i j}=\sum_{c=1}^{n} t_{i c} \otimes \bar{t}_{j c}, \quad i, j \in[1, m]
$$

by Section 6.3.1.

As a $\tilde{\mathcal{L}}\left(\mathrm{U}_{q}\left(\mathfrak{g l}_{m}\right)\right) \otimes \mathcal{L}\left(\mathrm{U}_{q}\left(\mathfrak{g l}_{m}\right)\right)$-module, $\mathcal{A}_{m}^{0}$ has the following decomposition into simples,

$$
\mathcal{A}_{m}^{0}=\bigoplus_{\lambda} L_{\lambda}^{(m)} \otimes L_{\lambda}^{(m)}
$$

where the summation is over all partitions of length $\leq m$. Following the proof of equation (6.9), one now shows that the $\tilde{\mathcal{L}}\left(\mathrm{U}_{q}\left(\mathfrak{g l}_{k}\right)\right) \otimes \mathcal{L}\left(\mathrm{U}_{q}\left(\mathfrak{g l}_{l}\right)\right)$ highest weight vectors of weight $\lambda$ in $\left(\mathcal{A}_{k, l}\right)^{\mathrm{U}_{q}\left(\mathfrak{g l}_{n}\right)}$ are precisely the $\tilde{\mathcal{L}}\left(\mathrm{U}_{q}\left(\mathfrak{g l}_{m}\right)\right) \otimes \mathcal{L}\left(\mathrm{U}_{q}\left(\mathfrak{g l}_{m}\right)\right)$ highest 
weight vectors of the same weight in $\mathcal{A}_{m}^{0}$. Hence $\mathcal{A}_{m}^{0}$ generates $\left(\mathcal{A}_{k, l}\right)^{\mathrm{U}_{q}\left(\mathfrak{g l}_{n}\right)}$ as a $\tilde{\mathcal{L}}\left(\mathrm{U}_{q}\left(\mathfrak{g l}_{m}\right)\right) \otimes \mathcal{L}\left(\mathrm{U}_{q}\left(\mathfrak{g l}_{m}\right)\right)$-module. That is,

$$
\left(\mathcal{A}_{k, l}\right)^{\mathrm{U}_{q}\left(\mathfrak{g l}_{n}\right)}=\left(\tilde{\mathcal{L}}\left(\mathrm{U}_{q}\left(\mathfrak{g l}_{k}\right)\right) \otimes \mathcal{L}\left(\mathrm{U}_{q}\left(\mathfrak{g l}_{l}\right)\right)\right) \mathcal{A}_{m}^{0} .
$$

To complete the proof of Theorem 6.10 in this case, we now need to show that every element of $\left(\tilde{\mathcal{L}}\left(\mathrm{U}_{q}\left(\mathfrak{g l}_{k}\right)\right) \otimes \mathcal{L}\left(\mathrm{U}_{q}\left(\mathfrak{g l}_{l}\right)\right)\right) \mathcal{A}_{m}^{0}$ can be expressed as a linear combination of products of $\Psi_{i \beta}(i \leq k, \beta \leq l)$. We shall prove this by induction on the bi-degree in the $\mathbb{Z}_{+} \times \mathbb{Z}_{+}$-grading of $\left(\mathcal{A}_{k, l}\right)^{\mathrm{U}_{q}\left(\mathfrak{g l}_{n}\right)}$. It is clear for the subspaces of bi-degrees $(0,0),(1,0)$ and $(0,1)$.

Now if $\zeta=\sum_{r} f_{r} \otimes g_{r}$ and $\zeta^{\prime}=\sum_{s} f_{s}^{\prime} \otimes g_{s}^{\prime}$ are elements of $\left(\mathcal{A}_{k, l}\right)^{\mathrm{U}_{q}\left(\mathfrak{g l}_{n}\right)}$, it follows from the commutativity of the left and right translations, that for all $x \in \mathrm{U}_{q}\left(\mathfrak{g l}_{k}\right)$ and $y \in \mathrm{U}_{q}\left(\mathfrak{g l}_{l}\right)$,

$$
\left(\tilde{\mathcal{L}}_{x} \otimes \mathcal{L}_{y}\right)\left(\zeta \zeta^{\prime}\right)=\sum_{(x),(y)}\left(\sum_{r} \tilde{\mathcal{L}}_{x_{(1)}}\left(f_{r}\right) \otimes \mathcal{L}_{y_{(1)}}\left(g_{r}\right)\right)\left(\sum_{s} \tilde{\mathcal{L}}_{x_{(2)}}\left(f_{s}^{\prime}\right) \otimes \mathcal{L}_{y_{(2)}}\left(g_{s}^{\prime}\right)\right) .
$$

This formula allows us to complete the induction step. The proof of Theorem 6.10 is now complete.

6.4. The braided exterior algebra. Let $V^{(m)}$ and $V^{(n)}$ be the natural modules for $\mathrm{U}_{q}\left(\mathfrak{g l}_{m}\right)$ and $\mathrm{U}_{q}\left(\mathfrak{g l}_{n}\right)$ respectively. Then $V^{(m)} \otimes V^{(n)}$ is an irreducible $\mathrm{U}_{q}\left(\mathfrak{g l}_{m} \times \mathfrak{g l}_{n}\right)$ module, and in this section, we shall study the braided exterior algebra of this module. Throughout this section, we denote $\mathfrak{g l}_{m} \times \mathfrak{g l}_{n}$ by $\mathfrak{g}$ and $\mathrm{U}_{q}\left(\mathfrak{g l}_{m} \times \mathfrak{g l}_{n}\right)$ by $\mathrm{U}_{q}(\mathfrak{g})$.

Denote the standard weight basis of the natural $\mathrm{U}_{q}\left(\mathfrak{g l}_{m}\right)$-module $V^{(m)}$ by $\left\{v_{i}^{(m)} \mid\right.$ $i=1,2, \ldots, m\}$. The tensor square $V^{(m)} \otimes V^{(m)}$ has two irreducible submodules, which we denote by $L^{(m, s)}$ and $L^{(m, a)}$ respectively, and which have the following bases.

(1) basis for $L^{(m, s)}$ :

$$
v_{i}^{(m)} \otimes v_{i}^{(m)}(\text { for all } i), \quad v_{i}^{(m)} \otimes v_{j}^{(m)}+q \otimes v_{j}^{(m)} \otimes v_{i}^{(m)}(i<j) ;
$$

(2) basis for $L^{(m, a)}$ :

$$
v_{i}^{(m)} \otimes v_{j}^{(m)}-q^{-1} \otimes v_{j}^{(m)} \otimes v_{i}^{(m)}(i<j) .
$$

Denote by $P^{(m, s)}$ and $P^{(m, a)}$ respectively the idempotent projections mapping $V^{(m)} \otimes$ $V^{(m)}$ onto the irreducible modules $L^{(m, s)}$ and $L^{(m, a)}$ respectively. Then the $R$-matrix of $\mathrm{U}_{q}\left(\mathfrak{g l}_{m}\right)$ acting on $V^{(m)} \otimes V^{(m)}$ is given by

$$
\check{R}^{\left(\mathfrak{g l}_{m}\right)}=q P^{(m, s)}-q^{-1} P^{(m, a)} .
$$

Similarly we have the natural $\mathrm{U}_{q}\left(\mathfrak{g l}_{n}\right)$-module $V^{(n)}$, with standard basis $\left\{v_{i}^{(n)} \mid\right.$ $i=1,2, \ldots, n\}$, and an analogous decomposition of its tensor square $V^{(n)} \otimes V^{(n)} \cong$ $L^{(n, s)} \oplus L^{(n, a)}$. Let $P^{(n, s)}$ and $\left.P^{(n, a)}\right)$ be the corresponding idempotent projections as above.

Write $V=V^{(m)} \otimes V^{(n)}$; this space has basis

$$
\left\{X_{i j}:=v_{i}^{(m)} \otimes v_{j}^{(n)} \mid i=1,2, \ldots, m, j=1,2, \ldots, n\right\} .
$$


Define the map $\sigma: V \otimes V \longrightarrow V^{(m)} \otimes V^{(m)} \otimes V^{(n)} \otimes V^{(n)}, v_{i}^{(m)} \otimes v_{j}^{(n)} \otimes v_{s}^{(m)} \otimes v_{t}^{(n)} \mapsto$ $v_{i}^{(m)} \otimes v_{s}^{(m)} \otimes v_{j}^{(n)} \otimes v_{t}^{(n)}$. Then the $R$-matrix of $\mathrm{U}_{q}(\mathfrak{g})=\mathrm{U}_{q}\left(\mathfrak{g l}_{m} \times \mathfrak{g l}_{n}\right)$ acting on $V \otimes V$ can be expressed as

$$
\check{R}=\sigma^{-1}\left(q^{2} P^{(m, s)} \otimes P^{(n, s)}+q^{-2} P^{(m, a)} P^{(n, a)}-P^{(m, s)} \otimes P^{(n, a)}-P^{(m, a)} \otimes P^{(n, s)}\right) \sigma .
$$

It follows that $\left(\check{R}-q^{2}\right)\left(\check{R}-q^{-2}\right)(\check{R}+1)=0$. Let

$$
\begin{aligned}
& a_{2}=\left(\check{R}-q^{2}\right)\left(\check{R}-q^{-2}\right)(V \otimes V)=\sigma\left(L^{(m, a)} \otimes L^{(n, s)}\right)+\sigma\left(L^{(m, s)} \otimes L^{(n, a)}\right), \\
& s_{2}=(\check{R}+1)(V \otimes V)=\sigma\left(L^{(m, s)} \otimes L^{(n, s)}\right)+\sigma\left(L^{(m, a)} \otimes L^{(n, a)}\right) .
\end{aligned}
$$

Now the braided symmetric algebra $S_{q}(V)$ of $V$ is known to be isomorphic to $\mathcal{M}_{m, n}$, which has been studied extensively [33, 3]. Here we consider the braided exterior algebra $\Lambda_{q}(V)$ defined (in analogy with the definition of $S_{q}(V)$ ) by

$$
\Lambda_{q}(V)=T(V) /\left\langle s_{2}\right\rangle,
$$

where $\left\langle s_{2}\right\rangle$ is the two-sided ideal in the tensor algebra $T(V)$ of $V$ generated by $s_{2}$.

Proposition 6.14. The associative algebra $\Lambda_{q}(V)$ is generated by

$$
\left\{X_{i j} \mid i=1,2, \ldots, m, j=1,2, \ldots, n\right\},
$$

subject to the following relations:

$$
\begin{aligned}
& X_{i l} X_{j k}+X_{j k} X_{i l}+\left(q-q^{-1}\right) X_{j l} X_{i k}=0, \\
& X_{i k} X_{j l}+X_{j l} X_{i k}=0, \quad i<j, \quad k<l, \\
& \left(X_{i k}\right)^{2}=0, \quad \forall i, k, \\
& X_{i l} X_{i k}+q^{-1} X_{i k} X_{i l}=0, \quad k<l, \quad \text { all } i, \\
& X_{j k} X_{i k}+q^{-1} X_{i k} X_{j k}=0, \quad i<j, \quad \text { all } k .
\end{aligned}
$$

Proof. Note that from $\sigma\left(L^{(m, a)} \otimes L^{(n, a)}\right)$, we obtain the relation

$$
X_{i k} X_{j l}+q^{-2} X_{j l} X_{i k}-q^{-1}\left(X_{j k} X_{i l}+X_{i l} X_{j k}\right)=0, \quad i<j, \quad k<l .
$$

The subspace $\sigma\left(L^{(m, s)} \otimes L^{(n, s)}\right)$ of the space of relations includes the relations (6.21), as well as

$$
X_{i k} X_{j l}+q^{2} X_{j l} X_{i k}+q\left(X_{j k} X_{i l}+X_{i l} X_{j k}\right)=0, \quad i<j, k<l .
$$

The relations (6.22) and (6.23) are equivalent to (6.20).

Proposition 6.15. The set $\{X(\epsilon)\}$ constitutes a basis of the braided exterior algebra $\Lambda_{q}(V)$, where $\epsilon$ runs over sequences of the form $\epsilon=\left(\epsilon_{1}, \epsilon_{2}, \ldots, \epsilon_{m n}\right)$, with $\epsilon_{i}=$ 0 or \pm 1 for $i=1, \ldots, m n$ and $X(\epsilon)=$ $\left(X_{11}\right)^{\epsilon_{11}}\left(X_{12}\right)^{\epsilon_{12}} \ldots\left(X_{1 n}\right)^{\epsilon_{1 n}}\left(X_{21}\right)^{\epsilon_{21}}\left(X_{22}\right)^{\epsilon_{22}} \ldots\left(X_{2 n}\right)^{\epsilon_{2 n}} \ldots\left(X_{m 1}\right)^{\epsilon_{m 1}} \ldots\left(X_{m n}\right)^{\epsilon_{m n}}$.

Proof. The fact that these elements span $\Lambda_{q}(V)$ is a consequence of Proposition 6.14. To prove that they are linearly independent, we consider the Grassmann algebra $\mathcal{K}[\theta]$ over $\mathcal{K}$. This is generated by $\left\{\theta_{i j}(1 \leq i \leq m ; 1 \leq j \leq n\}\right)$ with relations

$$
\theta_{i j} \theta_{k l}+\theta_{k l} \theta_{i j}=0, \text { for all } i, j, k, l \text {. }
$$


Given an $m n$ tuple $\epsilon=\left(\epsilon_{11}, \ldots, \epsilon_{1 n}, \epsilon_{21}, \ldots, \epsilon_{2 n}, \ldots, \epsilon_{m 1}, \ldots, \epsilon_{n m}\right)$ with $\epsilon_{i j}=0$ or 1 , write

$$
\theta(\epsilon)=\left(\theta_{11}\right)^{\epsilon_{11}} \ldots\left(\theta_{1 n}\right)^{\epsilon_{1 n}}\left(\theta_{21}\right)^{\epsilon_{21}} \ldots\left(\theta_{2 n}\right)^{\epsilon_{2 n}} \ldots\left(\theta_{m 1}\right)^{\epsilon_{m 1}} \ldots\left(\theta_{m n}\right)^{\epsilon_{m n}} .
$$

It is well known that the elements $\theta(\epsilon)$ form a basis of $\mathcal{K}[\theta]$.

Define an action of $\Lambda_{q}(V)$ on $\mathcal{K}[\theta]$ by recursion on degree as follows. First we specify the action on lower degree subspaces in the following way.

- Let

$$
X_{i k}(1)=\theta_{i k}, \quad \forall i, k
$$

- then define the action of $X_{j k}$ on $\theta(\epsilon)$ as follows:

(1) If for all $a<j$ and $t>k$, we have $\epsilon_{a t}=0$, then

$$
X_{j k} \theta(\epsilon)=(-1)^{\sum_{a=1}^{j} \sum_{t=1}^{k} \epsilon_{a t}} q^{-\sum_{a=1}^{j-1} \epsilon_{a k}} \theta\left(\epsilon+\mathbf{1}_{j k}\right),
$$

where $\mathbf{1}_{i k}$ denotes the $m n$ tuple whose $(i, k)$ entry is 1 and all of whose other entries are zero.

(2) Otherwise, let $i<j$ be the smallest integer such that there exists some $t>k$ with $\epsilon_{i t}=1$, and let $l$ be the smallest such $t$. Let

$$
\begin{aligned}
\epsilon_{<} & =\left(\epsilon_{11}, \ldots, \epsilon_{1 n}, \epsilon_{21}, \ldots \epsilon_{2 n}, \ldots, \epsilon_{i 1}, \ldots, \epsilon_{i l}, 0, \ldots, 0\right), \\
\epsilon_{>} & =\epsilon-\epsilon_{<} .
\end{aligned}
$$

Define

$$
\begin{aligned}
X_{j k} \theta(\epsilon) & =(-1)^{\sum_{a=1}^{i} \sum_{t=1}^{k} \epsilon_{a t}} q^{-\sum_{a=1}^{i} \epsilon_{a k}}\left[\theta\left(\epsilon_{<}\right)\left(X_{j k} \theta\left(\epsilon_{>}\right)\right)\right. \\
& \left.+\left(q-q^{-1}\right) \theta\left(\epsilon_{<}+\mathbf{1} n_{i k}-\mathbf{1}_{i l}\right)\left(X_{j l} \theta\left(\epsilon_{>}\right)\right)\right] .
\end{aligned}
$$

Note that the degree of $\theta\left(\epsilon_{>}\right)$is smaller than that of $\theta(\epsilon)$, so that by induction, the action of $X_{j k}$ and $X_{j l}$ on it are already defined. Therefore $X_{j k} \theta\left(\epsilon_{>}\right)$and $X_{j l} \theta\left(\epsilon_{>}\right)$are well defined elements in $\mathcal{K}[\theta]$.

The construction guarantees that the defining relations of $\Lambda_{q}(V)$ are satisfied. But clearly

$$
X(\epsilon)(1)=\theta(\epsilon)
$$

and the linear independence of the elements $X(\epsilon)$ follows from that of the $\theta(\epsilon)$ in the Grassmann algebra $\mathcal{K}[\theta]$.

The following result is a generalisation to the quantum group setting of [14, Theorem 4.1.1] known as 'skew $\left(G L_{m}, G L_{n}\right)$ duality' in the terminology of Howe.

Theorem 6.16. Let $V^{(m)}$ and $V^{(n)}$ be the natural modules for $\mathrm{U}_{q}\left(\mathfrak{g l}_{m}\right)$ and $\mathrm{U}_{q}\left(\mathfrak{g l}_{n}\right)$ respectively, and set $V=V^{(m)} \otimes V^{(n)}$. Then as a $\mathrm{U}_{q}\left(\mathfrak{g l}_{m}\right) \otimes \mathrm{U}_{q}\left(\mathfrak{g l}_{n}\right)$-module, the braided exterior algebra $\Lambda_{q}(V)$ decomposes into a multiplicity free direct sum of irreducibles as follows.

$$
\Lambda_{q}(V)=\bigoplus_{\lambda} L_{\lambda}^{(m)} \otimes L_{\lambda^{\prime}}^{(n)}
$$

where $\lambda^{\prime}$ denotes the conjugate of the partition $\lambda$, and the sum is over all partitions $\lambda=\left(\lambda_{1}, \lambda_{2}, \ldots, \lambda_{m}\right)$ such that $\lambda_{1} \leq n$.

The proof of the theorem will make use of the following result. 
Lemma 6.17. For any partition $\lambda=\left(\lambda_{1}, \lambda_{2}, \ldots, \lambda_{m}\right)$ with $\lambda_{1} \leq n$, let

$$
\Pi_{\lambda}:=X_{11} \ldots X_{1 \lambda_{1}} X_{21} \ldots X_{2 \lambda_{2}} \ldots X_{m 1} \ldots X_{m \lambda_{m}} \in \Lambda_{q}(V)
$$

Then

(1) The element $\Pi_{\lambda}$ is a highest weight vector with respect to the actions of $\mathrm{U}_{q}\left(\mathfrak{g l}_{m}\right)$ and of $\mathrm{U}_{q}\left(\mathfrak{g l}_{n}\right)$.

(2) The $\mathrm{U}_{q}\left(\mathfrak{g l}_{m}\right)$ weight of $\Pi_{\lambda}$ is $\lambda$, while the $\mathrm{U}_{q}\left(\mathfrak{g l}_{n}\right)$ weight is $\lambda^{\prime}$.

Proof. It is understood that if $\lambda_{i}=0$, then $\Pi_{\lambda}$ contains no $X_{i t}$ as a factor. This also implies that there is no $X_{j t}$ factor in $\Pi_{\lambda}$ for all $j \geq i$.

If $\lambda_{i+1}=0$, then the Chevalley generator $e_{i} \in \mathrm{U}_{q}\left(\mathfrak{g l}_{m}\right)$ acts on $\Pi_{\lambda}$ as 0 . Assume $\lambda_{i+1}>0$. Then $e_{i} \Pi_{\lambda}$ is a linear combination of terms of the form

$$
\Pi_{\nu} X_{i 1} \ldots X_{i \lambda_{i}} X_{i+1,1} \ldots X_{i+1, t-1} X_{i t} X_{i+1, t+1} \ldots X_{i+1, \lambda_{i+1}} \ldots X_{m 1} \ldots X_{m \lambda_{m}}
$$

where $\nu=\left(\lambda_{1}, \ldots, \lambda_{i-1}, 0, \ldots, 0\right)$ and $t \leq \lambda_{i+1}$. We prove that this is equal to zero by proving the more general result that for all $t$ such that $\lambda_{i} \geq t>s$,

$$
X_{i 1} X_{i 2} \ldots X_{i \lambda_{i}} X_{i+1,1} X_{i+1,2} \ldots X_{i+1, s} X_{i, t}=0 .
$$

If $s=0$, then using the second relation of (6.21) we can shift $X_{i t}$ to the left to arrive at the expression

$$
(-q)^{\lambda_{i}-t} X_{i 1} X_{i 2} \ldots X_{i t} X_{i t} X_{i, t+1} \ldots X_{i \lambda_{i}}
$$

which is zero by the first relation of (6.21). We now use induction on $s$. By (6.20), we may write $X_{i+1, s} X_{i, t}=-X_{i, t} X_{i+1, s}+\left(q-q^{-1}\right) X_{i s} X_{i+1, t}$. Thus

$$
\begin{aligned}
& X_{i 1} X_{i 2} \ldots X_{i \lambda_{i}} X_{i+1,1} X_{i+1,2} \ldots X_{i+1, s} X_{i, t} \\
& =X_{i 1} X_{i 2} \ldots X_{i \lambda_{i}} X_{i+1,1} X_{i+1,2} \ldots X_{i+1, s-1}\left(-X_{i, t} X_{i+1, s}+\left(q-q^{-1}\right) X_{i s} X_{i+1, t}\right),
\end{aligned}
$$

from which (6.24) follows by the induction hypothesis.

The fact that $\Pi_{\lambda}$ is also a $\mathrm{U}_{q}\left(\mathfrak{g l}_{n}\right)$ highest weight vector is proved similarly, by applying the Chevalley generators $e_{i} \in \mathrm{U}_{q}\left(\mathfrak{g l}_{n}\right)$ to $\Pi_{\lambda}$ and using the first and second relations of (6.21).

The second part of the lemma is obvious.

Proof of Theorem 6.16. For any $\mathbb{Z}_{\geq 0}$-graded module $M$, let $M_{\leq k}$ denote the sum of the graded components of degree at most $k$. It follows from Lemma 6.17 that $\bigoplus_{\lambda} L_{\lambda}^{(m)} \otimes L_{\lambda^{\prime}}^{(n)}$ is a $\mathrm{U}_{q}(\mathfrak{g})$-submodule of $\Lambda_{q}(V)$, where $\mathfrak{g}=\mathfrak{g l}_{m} \times \mathfrak{g l}_{n}$. Let $\Lambda\left(\mathbb{C}^{m} \otimes \mathbb{C}^{n}\right)$ be the exterior algebra of $\mathbb{C}^{m} \otimes \mathbb{C}^{n}$ over $\mathbb{C}$, and denote by $|\lambda|$ the size of the partition $\lambda$, that is, the sum of its parts (recall we always assume that $\lambda_{1} \leq n$ ). Then by skew $\left(G L_{m}, G L_{n}\right)$ duality [14, Theorem 4.1.1], we have

$$
\sum_{|\lambda| \leq k} \operatorname{dim}_{\mathcal{K}}\left(L_{\lambda}^{(m)} \otimes L_{\lambda^{\prime}}^{(n)}\right)=\operatorname{dim}_{\mathbb{C}} \Lambda\left(\mathbb{C}^{m} \otimes \mathbb{C}^{n}\right)_{\leq k}
$$

since irreducible modules of $\mathfrak{g}$ and of $\mathrm{U}_{q}(\mathfrak{g})$ with the same highest weight have the same dimension. By Proposition 6.15, $\operatorname{dim}_{\mathbb{C}} \Lambda\left(\mathbb{C}^{m} \otimes \mathbb{C}^{n}\right)_{\leq k}=\operatorname{dim}_{\mathcal{K}} \Lambda_{q}(V)_{\leq k}$ for all $k$. Hence

$$
\operatorname{dim}_{\mathcal{K}} \Lambda_{q}(V)_{\leq k}=\sum_{|\lambda| \leq k} \operatorname{dim}_{\mathcal{K}}\left(L_{\lambda}^{(m)} \otimes L_{\lambda^{\prime}}^{(n)}\right), \quad \forall k .
$$

This completes the proof. 


\section{ACKNOWLEDGEMENT}

The authors thank the Australian Research Council and National Science Foundation of China for their financial support.

\section{REFERENCES}

[1] Andersen, H. H.; Polo, P.; Wen, K. X. "Representations of quantum algebras". Invent. Math. 104 (1991), no. 1, 1-59.

[2] M. Atiyah, R. Bott, V.K. Patodi, "On the heat equation and the index theorem", Invent. Math. 19 (1973), 279-330.

[3] A. Berenstein, S. Zwicknagl, "Braided symmetric and exterior algebras", Trans. Amer. Math. Soc. 360 (2008), no. 7, 3429-3472.

[4] Brown, Ken A.; Goodearl, Ken R. "Lectures on algebraic quantum groups", Advanced Courses in Mathematics. CRM Barcelona, Birkhuser Verlag, Basel, 2002.

[5] J. Brundan, "Dual canonical bases and Kazhdan-Lusztig polynomials", J. Algebra 306 (2006), no. $1,17-46$.

[6] A. Connes, "Noncommutative geometry." Academic Press (1994).

[7] de Concini, C.; Procesi, C. "A characteristic free approach to invariant theory", Advances in Math. 21 (1976), 330-354.

[8] Drinfeld, V. G. "Quantum groups". Proceedings of the International Congress of Mathematicians, Vol. 1, 2 (Berkeley, Calif., 1986), 798-820, Amer. Math. Soc., Providence, RI, 1987.

[9] J. Du, L. Scott, B. Parshall, "Quantum Weyl reciprocity and tilting modules", Comm. Math. Phys. 195 (1998), no. 2, 321-352.

[10] Goodearl, Kenneth R.; Lenagan, Thomas H.; Rigal, Laurent, "The first fundamental theorem of coinvariant theory for the quantum general linear group", Publ. Res. Inst. Math. Sci. 36 (2000), no. 2, 269-296.

[11] Goodearl, K. R.; Lenagan, T. H. "Quantized coinvariants at transcendental q", Hopf algebras in noncommutative geometry and physics, Lecture Notes in Pure and Appl. Math., 239, Dekker, New York, 2005, 155-165.

[12] Gover, A. R.; Zhang, R. B. "Geometry of quantum homogeneous vector bundles and representation theory of quantum groups. I." Rev. Math. Phys. 11 (1999), 533-552.

[13] Gurevich, D. I.; Pyatov, P. N.; Saponov, P. A. "Quantum matrix algebras of GL $(m \mid n)$-type: the structure of the characteristic subalgebra and its spectral parametrization", (Russian) Teoret. Mat. Fiz. 147 (2006), no. 1, 14-46; translation in Theoret. and Math. Phys. 147 (2006), no. $1,460-485$

[14] Howe, R., "Transcending classical invariant theory." J American Math Soc 2, (1989), 535552.

[15] Jens Carsten Jantzen, "Lectures on quantum groups", Graduate Studies in Mathematics, 6, American Mathematical Society, Providence, RI, 1996.

[16] Jimbo, M. "A $q$-analogue of $U(\mathfrak{g l}(N+1))$, Hecke algebra, and the Yang-Baxter equation". Lett. Math. Phys. 11 (1986), no. 3, 247-252.

[17] R. Leduc, A. Ram, "A ribbon Hopf algebra approach to the irreducible representations of centralizer algebras: the Brauer, Birman-Wenzl, and type A Iwahori-Hecke algebras", Adv. Math. 125 (1997), 1-94.

[18] G.I. Lehrer, R.B. Zhang, "Strongly multiplicity free modules for Lie algebras and quantum groups", J. Algebra 306 (2006), no. 1, 138-174.

[19] G.I. Lehrer, R.B. Zhang, "A Temperley-Lieb analogue for the BMW-algebra", Progress in Mathematics, Birkhauser, in press.

[20] Jean-Louis Loday, "Cyclic homology, Appendix E by Mara O. Ronco", Grundlehren der Mathematischen Wissenschaften [Fundamental Principles of Mathematical Sciences] 301, Springer-Verlag, Berlin, 1992.

[21] Yu. Manin, "Quantum groups and noncommutative geometry", Universit de Montral, Centre de Recherches Mathmatiques, Montreal, QC, 1988. 
[22] S. Montgomery, "Hopf algebras and their actions on rings", CBMS Regional Conference Series in Mathematics, Vol. 82, American Mathematical Society, Providence, RI, 1993.

[23] C. Procesi, "The invariant theory of $n \times n$ matrices", Advances in Math. 19 (1976), 306-381.

[24] A. Ram, H. Wenzl, "Matrix units for centralizer algebras", J. Algebra 145 (1992), 378-395.

[25] Reshetikhin, N. Yu.; Turaev, V. G. "Ribbon graphs and their invariants derived from quantum groups". Comm. Math. Phys. 127 (1990), no. 1, 1-26.

[26] Reshetikhin, N.; Turaev, V. G. "Invariants of 3-manifolds via link polynomials and quantum groups". Invent. Math. 103 (1991), no. 3, 547-597.

[27] Olivia Rossi-Doria, "A $\mathrm{U}_{q}(\operatorname{sl}(2))$-representation with no quantum symmetric algebra". Rend. Mat. Acc. Lincei s. 9, 10 (1999) 5-9.

[28] E. Strickland, "Classical invariant theory for the quantum symplectic group", Adv. Math. 123 (1996), no. 1, 78-90.

[29] H. Wenzl, "On tensor categories of Lie type $E_{N}, N \neq 9$ ". Adv. Math. 177 (2003), no. 1, 66-104.

[30] Weyl, H. "The classical groups. Their invariants and representations". Fifteenth printing. Princeton Landmarks in Mathematics. Princeton Paperbacks. Princeton University Press, Princeton, NJ, 1997.

[31] R. B. Zhang, M. D. Gould, A. J. Bracken, "From representations of the braid group to solutions of the Yang-Baxter equation", Nucl. Physics B (1991), no. 2-3, 625-652.

[32] R. B. Zhang, "Structure and representations of the quantum general linear supergroup." Commun. Math. Phys. 195 (1998) 525-547.

[33] R. B. Zhang, "Howe duality and the quantum general linear group", Proc. Amer. Math. Soc. 131 (2003), no. 9, 2681-2692.

[34] S. Zwicknagl, "R-matrix Poisson algebras and their deformations." Adv. Math. 220 (2009), no. $1,1-58$.

School of Mathematics and Statistics, University of Sydney, Sydney, Australia

E-mail address: gusl@maths.usyd.edu.au

Department of Mathematical Sciences, Tsinghua University, Beijing, China

E-mail address: hzhang@maths.usyd.edu.au

School of Mathematics and Statistics, University of Sydney, Sydney, Australia

E-mail address: rzhang@maths.usyd.edu.au 